\title{
Underwater Optical Wireless Communications, Networking, and Localization: A Survey
}

\author{
Nasir Saeed, Abdulkadir Celik, Tareq Y. Al-Naffouri, Mohamed-Slim Alouini \\ Computer, Electrical and Mathematical Sciences \& Engineering (CEMSE) Division, King Abdullah University of Science E Technology (KAUST), Thuwal, KSA.
}

\begin{abstract}
Underwater wireless communications can be carried out through acoustic, radio frequency (RF), and optical waves. Compared to its bandwidth limited acoustic and RF counterparts, underwater optical wireless communications (UOWCs) can support higher data rates at low latency levels. However, the severe aquatic channel conditions (e.g., absorption, scattering, turbulence, etc.) pose great challenges for UOWCs and significantly reduce the attainable communication ranges, which necessitates efficient networking and localization solutions. Therefore, we provide a comprehensive survey on the challenges, advances, and prospects of underwater optical wireless networks (UOWNs) from a layer by layer perspective which includes: 1) Physical layer issues including propagation characteristics, channel modeling, and modulation techniques 2) Data link layer problems covering link configurations, link budgets, performance metrics, and multiple access schemes; 3) Network layer topics containing relaying techniques and potential routing algorithms; 4) Transport layer subjects such as connectivity, reliability, flow and congestion control; 6) Application layer goals, and 7) Localization and its impacts on UOWN layers. Finally, we outline the open research challenges and point out the prospective directions for underwater optical wireless communications, networking, and localization studies.
\end{abstract}

Keywords:

Underwater sensor networks, optical wireless communication, networking, localization, cross-layer design, relaying, routing, positioning, acquisitioning, tracking

\section{Introduction}

According to a recent survey by the United States National Oceanic and Atmospheric Administration (NOAA), about 97 percent of the Earth's water covers the surface of the earth in the form of oceans [1]. The early study of oceans (oceanography) which includes acquiring the knowledge of ocean tides, currents, and waves extends back to tens of thousands of years. However, it was not until the late $18^{\text {th }}$ century that the British government announced an expedition to conduct appropriate oceans scientific investigations. The results of this expedition were published in 1882 as "Report Of The Scientific Results of the Exploring Voyage of H.M.S. Challenger during the years 1873-76." [2]. After this expedition a number of books have been published on modern oceanography which include "Geography of the Oceans [3]", "Handbuch der Ozeanographie [4]", "The Depths of the Oceans[5]", "The Oceans [6]", "The Sea [7]", and "Encyclopedia of Oceanography [8]". More recently, there has been a growing interest in exploring the underwater environment for numerous applications such as climate change, the study of oceanic animals, the monitoring of oil rigs, surveillance, and unmanned operations. All of these applications require a medium to communicate in the underwater environment and from this environment to the outside world. Therefore, the study of underwater wireless media has attracted considerable attention for underwater communications.

\footnotetext{
Corresponding Author: Nasir Saeed (mr.nasir.saeed@ieee.org)
}

Today, underwater wireless communications (UWCs) are implemented using communication systems based on acoustic waves, radio frequency (RF) waves, and optical waves. Underwater acoustic wireless communications (UAWCs) have been one of the most used UWC technologies as they can provide communication over rather long distances. In 1995, an UAWC system was proposed in [9] with a data rate of $40 \mathrm{kbps}$. In 1996, an $8 \mathrm{kbps}$ UAWC system was developed for a depth of $20 \mathrm{~m}$ and an horizontal distance of $13 \mathrm{~km}$ [10]. In 2005, a more high-speed UAWC system was proposed in [11] which achieves a data rate of $125 \mathrm{kbps}$ using a 32 quadrature amplitude modulation technique (QAM) with a symbol error rate of $10^{-4}$. Furthermore, a $60 \mathrm{kbps}$ UAWC system was demonstrated in [12] using 32 QAM which can support communications at the depth of $100 \mathrm{~m}$ and for a horizontal distance of $3 \mathrm{~km}$. Besides the theoretical and experimental research on UAWC systems, various acoustic modems are commercially available which can be used for underwater applications. Fig. 1 shows the average achievable distance of various commercial acoustic modems for underwater communications. We also tabulate the main specifications of off-the-shelf commercial acoustic modems in Table 1 .

However, acoustic waves still have many drawbacks including scattering, high delay due to the low propagation speeds, high attenuation, and low bandwidth. Moreover, acoustic signals generated by communication systems and high-power sound navigation and ranging (SONAR) devices have adverse impact on the underwater mammals and fishes. 


\begin{tabular}{|c|c|c|c|}
\hline \multicolumn{4}{|l|}{ Nomenclature } \\
\hline$A F$ & Amplify-and-forward & OFDM & Orthogonal frequency-division multiplexing \\
\hline ACO-OFDM & Asymmetrically clipped optical OFDM & OFDMA & Orthogonal FDMA \\
\hline$A U V$ & Autonomous underwater vehicles & OCDMA & Optical CDMA \\
\hline$B E R$ & Bit Error Rate & $O E O$ & Optical-electrical-optical \\
\hline$B D F$ & Bit-detect-and-forward & $O A M$ & Orbital angular momentum \\
\hline CS & Candidate set & $P A P R$ & Peak-to-average power ratio \\
\hline CSPA & Candidate selection and prioritization & PAT & Pointing, acquisitioning, and tracking \\
\hline CDMA & Code-division multiple access & $P 2 P$ & Point-to-point \\
\hline$D F$ & Decode-and-forward & $P 2 M$ & Point-to-multipoint \\
\hline DCO-OFDM & Direct current biased optical OFDM & $R T E$ & Radiative transfer equation \\
\hline$D P$ & Distance progress & RSS & Received signal strength \\
\hline$D L$ & Downlink & $R F$ & Radio Frequency \\
\hline FoV & Field of View & SectOR & Sector-based OR \\
\hline$F E C$ & Forward Error Correction & $R T T$ & Round trip time \\
\hline FSO & Free-space optical & $S B L$ & Short baseline \\
\hline FDMA & Frequency-division multiple access & SONAR & Sound navigation and ranging \\
\hline FoV & Field of view & $S D M$ & pace-division multiplexing \\
\hline$G P S$ & Global positioning system & SDMA & Space-division multiple access \\
\hline GODAE & Global Ocean Data Assim. Exper. & $S P F$ & Scattering phase function \\
\hline GIB & GPS intelligent buoy & SDNs & Software-defined networks \\
\hline$H G$ & Henyey-Greenstein & TOWC & Terrestrial optical wireless communication \\
\hline H-Haul & Horizontal haul & ToA & Time of arrival \\
\hline IoUTs & Internet of Underwater Things & TDoA & Time difference of arrival \\
\hline ICI & Intercell interference & TDMA & Time division multiple access \\
\hline ISI & Inter-symbol interference & $T C P$ & Transmission control protocol \\
\hline IFDMA & Interleaved FDMA & $T I R$ & Total internal reflection \\
\hline$I M / D D$ & Intensity modulation direct detection & $Q o S$ & Quality of service \\
\hline $\mathrm{Li}-\mathrm{Fi}$ & Light Fidelity & $Q A M$ & Quadrature amplitude modulation \\
\hline$L o S$ & Line of sight & $Q A M-O F D M$ & Quadrature amplitude modulation OFDM \\
\hline$L E D$ & Light emitting diode & $U W C$ & Underwater wireless communication \\
\hline$L A N$ & Local area network & $U A W C$ & Underwater acoustic wireless communication \\
\hline$L B L$ & Long baseline & UOWCs & Underwater optical wireless communications \\
\hline$M I$ & Magnetic induction & UOWN & Underwater optical wireless network \\
\hline$M F C s$ & Microbial fuel cells & UWSN & Underwater sensor network \\
\hline MAI & Multiple access interference & $U D P$ & User datagram protocol \\
\hline NOAA & National Oceanic and Atmosp. Admin. & $U L$ & Uplink \\
\hline$N L o S$ & Non line of sight & V-Haul & Vertical haul \\
\hline NOMA & Non-orthogonal multiple access & $V L C$ & Visible light communication \\
\hline OBSs & Optical base stations & $V S F$ & Volume scattering function \\
\hline$O R$ & Opportunistic routing & $W D M$ & Wavelength-division multiplexing \\
\hline OOK & On-off keying & WDMA & Wavelength-division multiple access \\
\hline
\end{tabular}




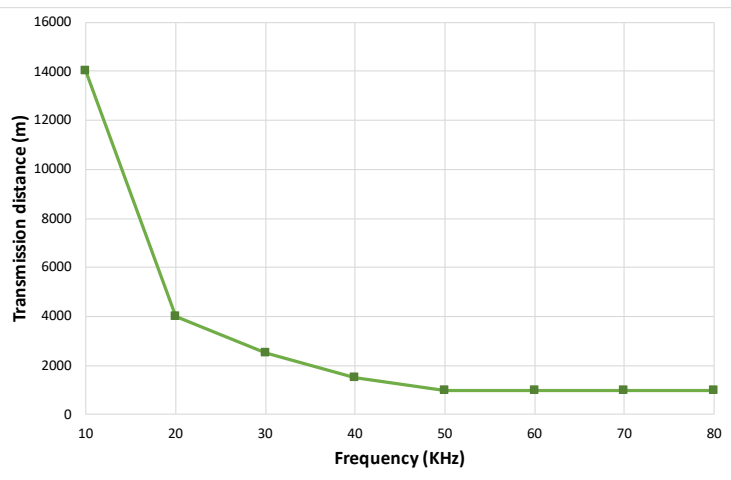

Figure 1: Average achievable transmission distance by various commercial acoustic modems

Table 1: Comparison of various commercial underwater acoustic modems.

\begin{tabular}{|l|l|l|l|}
\hline \hline Acoustic modem & Bandwidth & Data rate & Distance \\
\hline DSPComm AquaComm Mako [13] & $14 \mathrm{KHz}$ & $240 \mathrm{bps}$ & $100 \mathrm{~m}$ \\
\hline TriTech MicronModem [14] & $4 \mathrm{KHz}$ & $40 \mathrm{bps}$ & $500 \mathrm{~m}$ \\
\hline LinkQuestUWM10000 [15] & $5 \mathrm{KHz}$ & $5000 \mathrm{bps}$ & $1 \mathrm{~km}$ \\
\hline LinkQuestUWM1000 [15] & $17.8 \mathrm{KHz}$ & $17800 \mathrm{bps}$ & $3.5 \mathrm{~km}$ \\
\hline EvoLogics S2CR7/17 USBL [16] & $10 \mathrm{KHz}$ & $6900 \mathrm{bps}$ & $8 \mathrm{~km}$ \\
\hline Teledyne BenthosAtm88x [17] & $5 \mathrm{KHz}$ & $2400 \mathrm{bps}$ & $6 \mathrm{~km}$ \\
\hline GPM 3000Modem [18] & - & $1200 \mathrm{bps}$ & $25 \mathrm{~km}$ \\
\hline \hline
\end{tabular}

Although UAWC systems are suitable to provide command and control applications due to their long transmission range, their data rate is insufficient for underwater multi-media applications. Therefore, research is carried out in the past to use low-frequency RF waves, e.g., the authors in [19] proposed a microwave-based wireless communication system over the surface of the ocean water which can transmit data over tens of kilometers. An underwater microwaves-based wireless communication system was employed in [20], which can communicate over a horizontal distance of $85 \mathrm{~m}$. A similar approach was followed in [21] with a data rate of $500 \mathrm{kbps}$ over a horizontal distance of $90 \mathrm{~m}$. The authors in [22] have improved the capacity of underwater microwaves-based wireless communication systems further to $10 \mathrm{Mbps}$ over a distance of $100 \mathrm{~m}$. However, RF waves including microwaves suffer from serious attenuation in the water, e.g., the attenuation in the ocean is about $169 \mathrm{~dB} / \mathrm{m}$ for the $2.4 \mathrm{GHz}$ band while the attenuation in freshwater is even higher, i.e., $189 \mathrm{~dB} / \mathrm{m}$ [23]. Moreover, RF-based UWC requires huge antennas and are limited to the shallow areas of the sea. Even though operating at ultra-low frequencies yields reduced attenuation levels, this is achieved at the expense of high hardware costs and low data rates.

Due to the low bandwidth and low data rate limitations of underwater acoustic and RF waves, an alternative approach is to use optical waves which can provide high-speed underwater optical wireless communications (UOWC) at low latencies in return for a limited communication range. The underwater propagation of optical waves also exhibits distinctive characteristics in different wavelengths as shown in Fig. 2. In 1963, the authors in [24] found that attenuation within the range of 450-

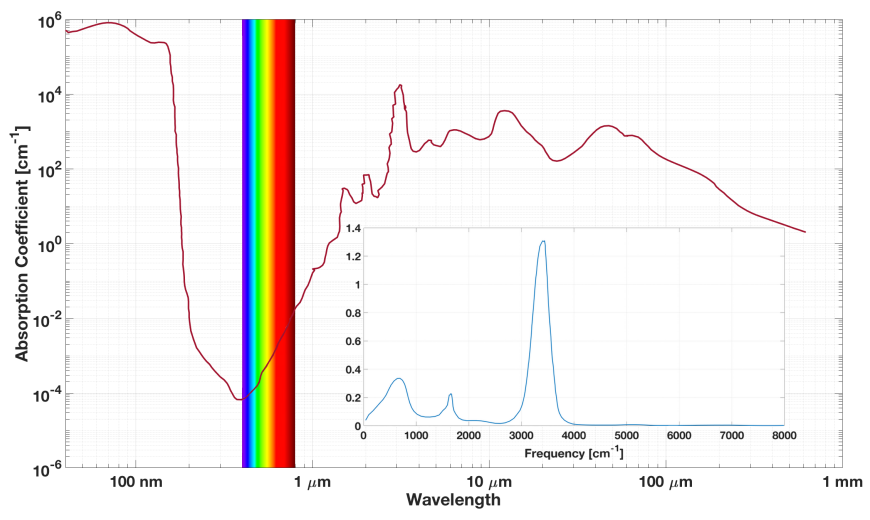

Figure 2: Attenuation of optical waves in the aquatic medium.

$550 \mathrm{~nm}$ wavelengths (blue and green lights) is much smaller compared to the other wavelengths, which is mainly because of the photosynthesis activity of algae especially during the warm seasons and in the coastal water. Hence, underwater optical communications and sensor systems for coastal applications are designed to operate in the green spectral range. In 1966, Gilbert et al. [25] experimentally confirmed this behavior of optical waves, which provided the foundation of UOWC systems. The research on UOWC is mainly focused on increasing the transmission range and the data rate of UOWC systems for different types of water. The trend to improve the data rate of UOWC systems by using light emitting diodes (LEDs) can be seen in [26, 27, 28, 29, 30, 31, 32].

All of the LED-based UOWC systems provide the data rate of Mbps in pure sea water where the transmission distance is restricted by the radiation angle and light intensity distribution. Hence, laser diode based UOWC systems are developed to provide longer transmission distance and higher data rates $[33,34,35]$. In [26], the authors conducted a lab experiment by using a green laser with $532 \mathrm{~nm}$ wavelength to provide a UOWC link which covers a distance of $2 \mathrm{~m}$ with a data rate of 1 Gbps. The authors in [36] performned a lab experiment with $405 \mathrm{~nm}$ wavelength blue laser to provide a 1.45 Gbps UOWC link with a transmission distance of $4.8 \mathrm{~m}$. To further improve the transmission distance and data rate, the authors in [37] and [38] conducted lab experiments to provide an UOWC link with $2.3 \mathrm{Gbps}$ and $2.488 \mathrm{Gbps}$ over a transmission distance of $7 \mathrm{~m}$ and $1 \mathrm{~m}$, respectively. Subsequently, the authors in [39] demonstrated a UOWC system with a data rate of 4.8 Gbps using 16 quadrature amplitude modulation-orthogonal frequency division multiplexing (QAM-OFDM) in clear water. A lab experiment was performed for turbid harbor water in [40] where the authors were able to achieve the data rate of $4.88 \mathrm{Gbps}$ by using 32 QAM-OFDM over a transmission distance of $6 \mathrm{~m}$. Recently, a 7.2 Gbps UOWC system was proposed in [41] for a $450 \mathrm{~nm}$ blue laser which was able to achieve the transmission distance of $6 \mathrm{~m}$ in seawater. Alternatively, hybrid systems comprising of both acoustic and optical underwater wireless communication system are introduced in [42, 43, 44, 45, 46] where acoustic waves are used for command and control applications and op- 
Table 2: Comparison of underwater wireless communication systems [47].

\begin{tabular}{|l|l|l|l|}
\hline \hline Parameters & RF & Acoustic & Optical \\
\hline Range & $<100 \mathrm{~m}$ & $<20 \mathrm{Km}$ & $100-200 \mathrm{~m}$ \\
Attenuation & $\begin{array}{l}\text { Frequency \& } \\
\text { Conductivity }\end{array}$ & $\begin{array}{l}\text { Conductivity } \\
\text { Factors }\end{array}$ & $\begin{array}{l}\text { Distance and } \\
\text { inherent opti- } \\
\text { cal properties } \\
2.25 \times 10^{8} \mathrm{~m} / \mathrm{s}\end{array}$ \\
Speed & $2.25 \times 10^{8} \mathrm{~m} / \mathrm{s}$ & $1500 \mathrm{~m} / \mathrm{s}$ & $\approx 1 \mathrm{~W}$ \\
Tx. Power & $\approx 100 \mathrm{~W}$ & $\approx 10 \mathrm{~W}$ & High \\
Cost & High & $<10 \mathrm{Kbps}$ & $<10 \mathrm{Gbps}$ \\
Data rate & $<0.1 \mathrm{Gbps}$ & $0.1 \mathrm{~m}$ & $0.1 \mathrm{~m}$ \\
Antenna size & $0.5 \mathrm{~m}$ & High & Low \\
Latency & Moderate & \multicolumn{3}{|l}{} \\
\hline \hline
\end{tabular}

tical waves are used for multi-media applications. However, the research on developing hybrid systems is still in its infancy and needs proper analysis. Table 2 summarizes the comparison between the three different kinds of underwater wireless communication systems.

\subsection{Related Surveys on UOWNS}

With the increasing demand for UOWN applications, a few brief surveys have been published to discuss the physical layer aspects of UOWNs. In [23], the authors discussed modulation schemes, channel models, link management, and coding techniques along with the possible practical implementations of UOWC systems. The link performance of UOWC systems was evaluated in [27] and various challenges associated with the link developments of UOWC systems were introduced. In [48], UOWC systems were reviewed in terms of modulation schemes, channel models, and coding schemes. The channel models of UOWC systems have also been surveyed in [49] and [50], where vector radiative transfer theory, variable water composition, and inherent properties of light were considered. The inherent features of underwater wireless communications, including UOWC, have been briefly surveyed in [47]. The recent advances in system analysis and channel modeling of UOWC systems have been summarized in [51]. In [52] the future vision of UOWC systems and some of its challenges were presented.

Although these surveys tackle various physical layer aspects of UOWNs, limited transmission ranges of UOWCs necessitates powerful and novel networking solutions to be implemented in real life. Furthermore, accurate and precise localization schemes are essential for developing effective networking protocols. We should also note that some types of applications heavily depend upon the sensing location since the obtained measurements are meaningful only if they refer to an accurate location. Therefore, this paper provides a comprehensive survey on the challenges, advances, and prospects of UOWNs from a layer by layer perspective which includes:

- Physical layer issues including propagation characteristics, channel modeling, and modulation techniques.

- Data link layer problems covering link configurations, link budgets, performance metrics, and multiple access schemes.

- Network layer topics containing relaying techniques and potential routing algorithms.
Table 3: Comparison of this paper with the existing surveys.

\begin{tabular}{|l|r|l|}
\hline \hline Ref. & Year & Area of Focus \\
\hline $\begin{array}{l}\text { Arnon } \text { et al. } \text { [27] } \\
\text { [49] }\end{array}$ & 2010 & UOWC Link configurations \\
\hline $\begin{array}{l}\text { Jhonsan et al. } \\
{[50]}\end{array}$ & 2013 & UOWC channel models \\
\hline Camila et al. [47] & 2014 & UOWC channel models \\
\hline $\begin{array}{l}\text { Kaushal et al. } \\
\text { [48] }\end{array}$ & 2016 & $\begin{array}{l}\text { Physical layer issues of UOWC such as } \\
\text { channel models, modulation schemes, link } \\
\text { management, and coding. }\end{array}$ \\
\hline $\begin{array}{l}\text { Zeng } \text { et al. } \text { [23] } \\
\text { This survey }\end{array}$ & 2017 & $\begin{array}{l}\text { Physical layer issues of UOWC such as } \\
\text { channel models, modulation schemes, link } \\
\text { management, and coding. }\end{array}$ \\
\hline $\begin{array}{l}\text { Potential UOWN architectures, a layer-by- } \\
\text { layer discussion of networking aspects, lo- } \\
\text { calization, and future research directions. }\end{array}$ \\
\hline
\end{tabular}

- Transport layer subjects such as connectivity, reliability, flow and congestion control.

- Application layer goals and state-of-the-art UOWNs applications.

- Localization and its impact on UOWNs layers.

- Outlining several open research challenges and future directions in UOWNs.

Table 3 summarizes the comparison of this paper with the existing surveys discussed above.

\subsection{Survey Organization}

The rest of this survey is organized as follows: Section 2, addresses the physical layer aspects of UOWNs such as underwater propagation characteristics of optical waves, channel modeling, and UOWC modulation techniques. The data link layer issues such as the fundamental tradeoff between transmission angle and range, link configurations, bit error rate and data rate performance, and multiple access schemes are covered in Section 3. Section 4 discusses network layer problems including relaying techniques and routing protocols. Section 5 covers the transport layers topics including connectivity, reliability, flow control, and congestion control in UOWNs. Application layer goals and a number of UOWN applications are presented in Section 6. Different localization techniques for UOWNs are presented in Section 7. Section 8 outlines open research challenges and points out future directions in UOWNs research. Finally, Section 9 concludes the survey with a few remarks.

\section{Physical (PHY) Layer: Essentials of UOWCs}

Before delving into the UOWNs layer by layer, it is important to provide an overview of potential architectures which can either be built in an ad hoc or in an infrastructure fashion. An ad hoc UOWN is a distributed type of wireless network which does not rely upon any pre-installed network equipment. Hence, traffic requests are carried out by the participation of nodes along a routing path which is dynamically determined 


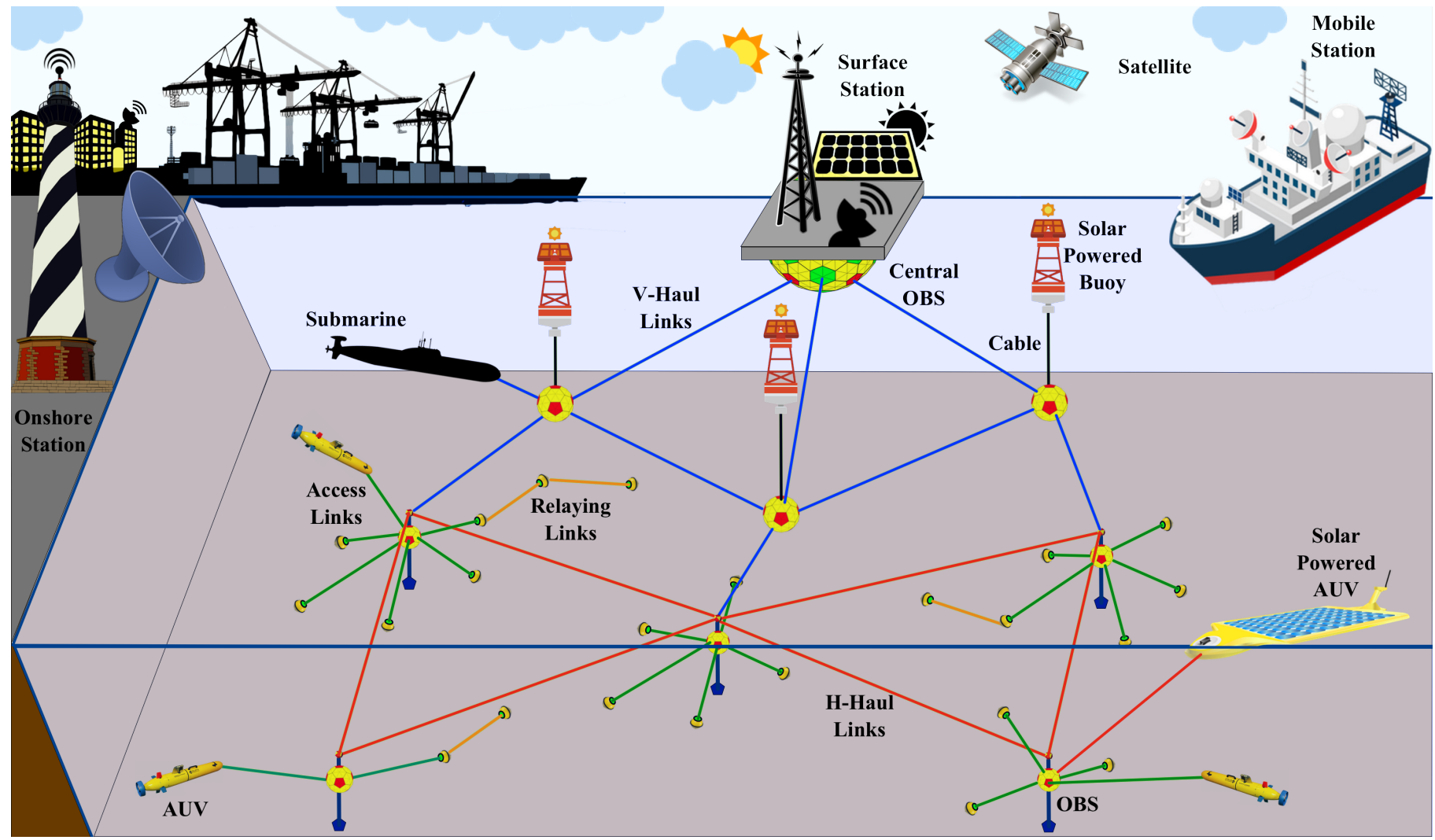

Figure 3: Illustration of a generic underwater optical wireless network (UOWN) architecture.

based on network connectivity, this approach may necessitate self-configuration and self-organization skills because of the absence of a central control unit. There are several potential connectivity challenges due to the directional light propagation with limited range, thus making the realization of a full ad-hoc UOWN a non-trivial engineering task. On the other hand, infrastructure-based UOWNs may consist of omnidirectional optical access points or optical base stations (OBSs) each of which creates an underwater local area network by serving and coordinating nodes in its vicinity or cell coverage area, respectively.

Fig. 3 shows a generic cellular infrastructure-based threedimensional architecture where the underwater sensor nodes communicate with each other and with the underwater OBSs by using optical waves presented by orange links and dark green links respectively. The communication between the OBSs at the same depth is presented in red colored optical links, i.e., horizontal haul (H-Haul) links, while the information from the OBSs which are at a greater depth is relayed to the central OBS at the surface station by the OBSs at the low depth, i.e., vertical haul (V-Haul) links are used, drawn in blue color. It is also shown in Fig. 3 that the surface buoys can operate on solar power thus improving the energy efficiency of the network. Moreover, submarines and autonomous underwater vehicles (AUVs) can also communicate with the OBSs by using the UOWC. Finally, the information gathered at the surface station can be transmitted to the onshore station or to a mobile station by using terrestrial RF networks. Note that the OBSs and AUVs in Fig. 3 can also be designed to operate on both optical and acoustic waves for reaping the full benefits of both prominent technologies. This way, optical systems can provide high-speed, low latency data links while acoustic systems can support ubiquitous control and command functions due to the long-range and omnidirectional nature [45]. Also, OBSs can be interconnected with fibers to realize the H-Haul links.

Physical layer is essential for many crucial communication functions including channel modeling and estimation, signal processing, modulation, and coding. Compared to the higher layers, the physical layer of UOWNs has been described and studied thoroughly. In this section, we start with a comparison on the virtues and drawbacks of the three main UWC systems: acoustic, radio frequency, and optical. Then, a detailed discussion of underwater propagation characteristics of optical waves is presented including absorption, scattering, turbulence, pointing, alignment, multipath fading, and delay spread.

\subsection{Waves Under the Sea: A Tour of the Underwater Commu- nications}

This section briefly introduces the different types of carrier waves used for underwater wireless communications technologies, which include acoustic waves, RF waves, and optical waves.

\subsubsection{Acoustic Waves}

UAWC systems are employed in almost every military and commercial application of UWC [53]. The most prominent 


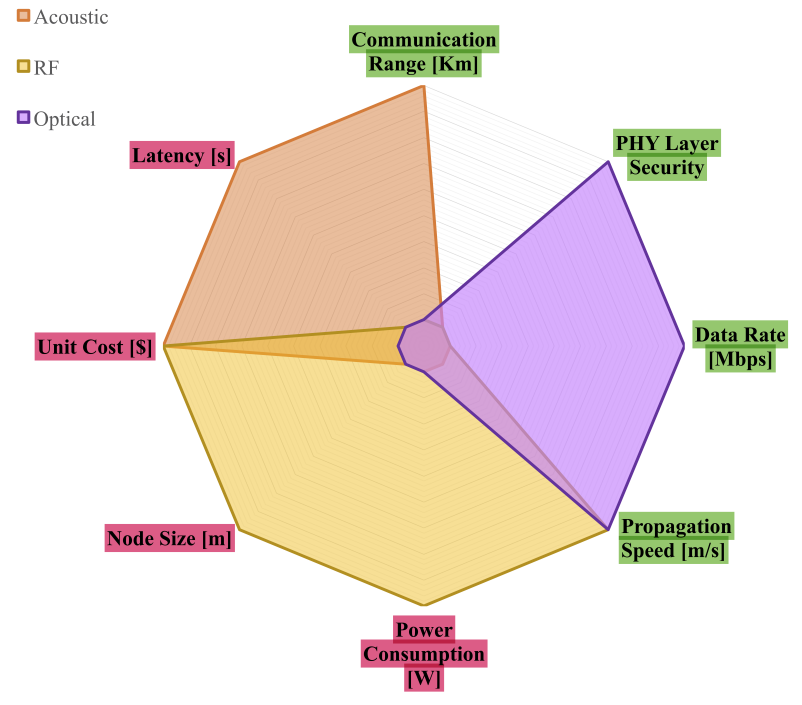

Figure 4: Comparison of acoustic, RF, and optical waves under different performance metrics which are highlighted with green and red for values preferred high and low, respectively. We should note that these parameters are open to interpretation depending on the exact environment and that represented values are illustrative and subjective.

feature of the acoustic systems is their ability to reach very long distances, up to tens of kilometers [54]. Nevertheless, UAWC systems cannot provide high quality services for underwater multi-media applications due to the following innate restrictions. The nominal propagation speed of the underwater acoustic signal is around $1500 \mathrm{~m} / \mathrm{s}$ which yields a latency in the order of seconds [55]. Hence, the delay performance of acoustic systems is not desirable for real-time telephony and multi-media applications. The operation bandwidth of underwater acoustic signals is between tens of Hertz to hundreds of $\mathrm{kHz}$ and the achievable data rates of acoustic links are typically in the order of kbps, which is apparently not adequate to sustain the transmission of large data volumes [56]. Additionally, the cost of acoustic nodes makes the creation of a large scale underwater acoustic network economically demanding. Moreover, its energy inefficiency may necessitate a battery replacement burden that can be quite a problematic task for nodes placed in the deep sea. Moreover, acoustic systems based on high-power SONARs can also distress marine mammals such as dolphins and whales [57].

\subsubsection{Radio Frequency Waves}

The exploitation of RF signals can provide a smooth transition between terrestrial and underwater communication systems $[58,20]$. Unlike acoustic waves, RF signals are more tolerant to the turbulence and turbidity effects of the water. Thus, they can provide a higher propagation speed [20]. However, the underwater RF communication is restricted to shallow waters and limited to the extremely low frequency band (i.e., $30-300 \mathrm{~Hz}$ ) which yields a limited data rate even at very short communication ranges [59]. For worldwide communications with submarines for depths up to a few 10 meters very low frequency (VLF) transmitters from $10-30 \mathrm{kHz}$ are used. Even if low- priced terrestrial RF modules can be integrated into a pennysize module, underwater RF nodes are costly, and require huge antennas, and high transmission power to compensate for the high antenna losses $[60,58]$.

\subsubsection{Optical Waves}

In comparison with the acoustic and RF systems, UOWC can support higher data rates over distances of tens of meters, which can reach up to several Gbps in clear waters with little to no scattering. Moreover, UOWC systems provide a low latency performance thanks to the high propagation velocity of light in the aquatic medium (i.e., $\approx 2.25 \times 10^{8} \mathrm{~m} / \mathrm{s}$ ) [61, 62, 63]. These two main advantages of optical waves can enable many realtime communication and control applications such as largescale UWSNs and video-surveillance via AUVs. Furthermore, underwater optical wireless transceivers can be built in small sizes with low-cost and energy-conservative laser and photodiodes. Besides, optical wireless communication generally takes place in a point-to-point fashion, and hence provides enhanced security in comparison with RF and acoustic underwater communications. However, directed nature of light propagation reduces the connectivity of the network and requires accurate pointing, acquisitioning, and tracking (PAT) mechanisms to sustain reliable links. Alternatively, optical transceivers with broad beams/omnidirectional propagation capabilities can be built to mitigate the PAT requirements at the cost of potentially less secure and low range communications [64].

Despite all these appealing virtues, there exist many challenges to implement UOWC systems in practice. Firstly, as it is the case for the free-space optical communication, a misalignment of the optical transceivers can cause short-term disconnections which are generally a result of random movements of the sea surface $[65,66]$, depth-depended variations, deep currents [49], and oceanic turbulence [67]. Secondly, even if the carrier wavelength of the light beam is chosen to be blue or green in order to mitigate the underwater attenuation effects [68, 24, 25], the light beam propagation still undergoes absorption, scattering, turbulance, and thus multipath fading because of the interactions of water molecules and particulates with the photons $[61,62]$. Such kind of impairments cause performance degradation and reduce the communication range significantly.

Table 2 compares these three technologies by tabulating the important state-of-the-art system parameters. For the sake of a better visualization, we also draw a radar chart in Fig. 4 to highlight the potential of UOWC systems which obviously exhibit a good performance in terms of data rate, propagation speed, power consumption, latency, cost, and size. However, the main limitation is set by the short communication ranges which definitely entails range expansion via networking of optical nodes in order to operate in a large area of interest. Furthermore, the misalignment of optical transceivers is one of the most challenging networking and control problems and necessitates precise alignment algorithms with inherited self-organization and self-configuration features to keep the nodes connected all the time. Therefore, it is of utmost importance to gain important insights into the UOWNs from a networking point of view including relaying, routing, deployment, localization, en- 


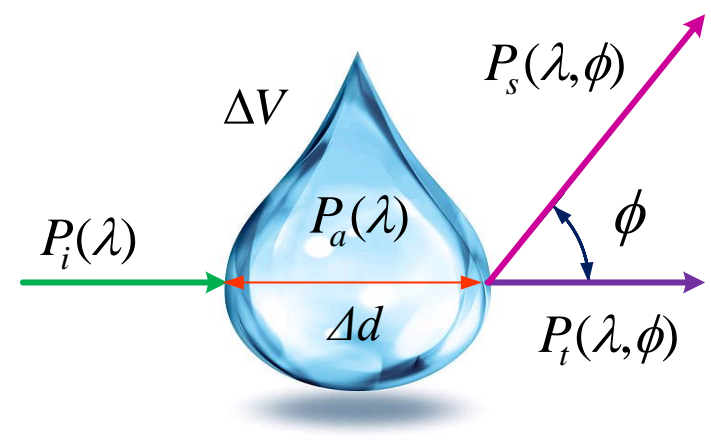

Figure 5: Geometric model for the inherent optical properties.

ergy harvesting, mobility, network lifetime maximization, selfconfiguration, and self-organization. Before proceeding to the higher layers of UOWNs, however, we believe it is necessary to briefly discuss the physical layer aspects for the sake of completeness of the survey. Accordingly, the following subsections address the propagation characteristics, and channel modeling in some depth as they are the building blocks of UOWNs.

\subsection{Underwater Propagation Characteristics and Channel Modeling of Optical Waves}

Underwater communication channels exhibit quite different propagation characteristics at different locations and depths since they vary with the physio-chemical nature of the Oceans. In particular, optical attributes of the aquatic medium are categorized based on inherent and apparent properties. While the inherent optical properties include absorption, scattering, and attenuation coefficients, which heavily depend on the chemical composition of seawater [69], the apparent optical properties consist of radiance, irradiance, and reflectance factors which are determined by the geometric parameters of the light beams (e.g., diffusion and collimation) [70]. In the remainder of this subsection, firstly, the underwater optical wireless attenuation, absorption, and scattering models are presented, including the Beer-Lambert law, the volume scattering function, the radiative transfer equation, and Monte-Carlo methods. Secondly, the oceanic turbulence models are discussed for UOWC channels and, finally, the models for pointing and misalignment are presented.

\subsubsection{Absorption $\mathcal{E}$ Scattering}

Absorption restricts the transmission range of an underwater optical wireless link by causing the total propagation energy of an emitted light beam to continuously decrease. On the other hand, scattering spreads the photons toward random directions such that some portion of them is not received by the receiver as it has a finite aperture size, whereas the reception of some other portions may be delayed due to the following of different propagation paths. Thus, scattering leads to multi-path fading, time-jitter, and inter-symbol interference phenomena. The volume scattering function (VSF) can be interpreted as the scattered intensity per unit incident irradiance per unit volume of water and expressed as [71]:

$$
\vartheta(\lambda, \phi)=\lim _{\Delta d \rightarrow 0} \lim _{\Delta \omega \rightarrow 0} \frac{P_{s}(\lambda, \phi)}{\Delta d \Delta \omega}
$$

where $P_{s}(\lambda, \phi)$ is the power of the scattered light beam into a solid angle which is centered on $\phi$ as shown in Fig. 5. Hence, the scattering coefficient can be obtained by integrating VSF over all the directions, i.e., $b(\lambda)=\int \vartheta(\lambda, \phi) d \omega$. Furthermore, the scattering phase function (SPF) can be expressed by normalizing the VSF by the scattering coefficient [71], i.e., $\tilde{\vartheta}(\lambda, \phi)=\frac{\vartheta(\lambda, \phi)}{b(\lambda)}$, which is commonly represented by the Henyey-Greenstein (HG) phase function [72, 63, 61]. HG phase function is a convenient approximation of the ocean scattering functions. They are helpful in reducing complexity in the calculations, but can significantly influence the delay spread $[73,34]$.

Extinction coeffceint which is the sum of absorption and scattering coefficients can be formulated based on a geometric model proposed in [74], given as

$$
c(\lambda)=a(\lambda)+b(\lambda)
$$

where $a(\lambda), b(\lambda)$, and $c(\lambda)$ are in units of $m^{-1}$. These coefficients heavily depend on water types and depths. Based on their influence on the inherent optical properties, the oceanic water types are classified by Petzold as follows [75]:

- Pure sea water: Pure sea water consists of pure water molecules $\left(\mathrm{H}_{2} \mathrm{O}\right)$ and dissolved salts $\left(\mathrm{NaCl}, \mathrm{MgCl}_{2}\right.$, $\mathrm{Na}_{2} \mathrm{SO}_{4}, \mathrm{KCl}$, etc.), whose absorption effect sum mainly determines the total absorption in the pure sea water. As the scattering coefficient of the pure sea water is negligible [76], the light beam propagates in a straight line with very limited dispersion. In pure seawater $a(\lambda)=0.053 \mathrm{~m}^{-1}$, $b(\lambda)=0.003 \mathrm{~m}^{-1}$, and $c(\lambda)=0.056 \mathrm{~m}^{-1}$ respectively.

- Coastal ocean water: The coastal Ocean waters are highly concentrated due to the dissolved particles, thus, they display more severe absorption and scattering effects. Hence, $a(\lambda)=0.179 \mathrm{~m}^{-1}, b(\lambda)=0.219 \mathrm{~m}^{-1}$, and $c(\lambda)=0.398 \mathrm{~m}^{-1}$.

- Turbid harbor water: The turbid harbor water has the highest concentration of suspended and dissolved particles and therefore has high absorption, scattering, and extinction coefficients with $a(\lambda)=0.295 \mathrm{~m}^{-1}, b(\lambda)=1.875 \mathrm{~m}^{-1}$, and $c(\lambda)=2.17 \mathrm{~m}^{-1}$ respectively.

Extending from the sea surface to the bottom, the chlorophyll variation curve follows a skewed Gaussian profile [50]. Accordingly, the attenuation coefficient starts from $0.05 \mathrm{~m}^{-1}$ and reaches the peak value of $0.1 \mathrm{~m}^{-1}$ around the $100 \mathrm{~m}$ depth, which starts decreasing again for deeper waters [49]. In what follows, we discuss the various attenuation, absorption, and scattering models for UOWC systems.

Beer-Lambert Law. The simplest and, thus, most widely used model to describe the UOWC channel attenuation is the BeerLambert Law which expresses the received signal power at the 
receiver as:

$$
P_{r}(\lambda, d)=P_{t} e^{-c(\lambda) d},
$$

where $P_{t}$ is the transmission power of the transmitter, $c(\lambda)$ is the extinction coefficient given in (2) and $d$ is the Euclidean distance between the transceivers. As previously discussed in detail, $c(\lambda)$ changes for different water types and depths [77, 78]. For practical values of $c(\lambda)$, we refer the interested readers to the works in $[49,71,74,79,80,50,81,26]$. Assuming a perfect pointing between the transceivers, the Beer-Lambert Law presumes that all the scattered photons are lost by ignoring the multipath arrival of the scattered photons. Therefore, Beer-Lambert law overestimates the amount of attenuation because of the collection of forward scattered light. As discussed in [41], multiple scattering induces high packet losses for higher order modulation schemes. To overcome this deficiency, more sophisticated models are proposed. These models are introduced in the following subsections.

Radiative Transfer Equation. RTE can describe the energy conservation of a light beam passing through a steady medium [82], which is expressed as [83, 84]:

$$
\begin{aligned}
\vec{r} \cdot \nabla L(\lambda, \vec{r}, \vec{\ell})= & -c L(\lambda, \vec{r}, \vec{\ell})+\int_{2 \pi} \vartheta\left(\lambda, \vec{r}, \vec{r}^{\prime}\right) L(\lambda, \vec{r}, \vec{\ell}) d \vec{r}^{\prime} \\
& +E(\lambda, \vec{r}, \vec{\ell})
\end{aligned}
$$

where $\vec{r}$ is the direction vector, $\nabla$ is the divergence operator, $L(\lambda, \vec{r}, \vec{\ell})$ represents the optical radiance at position $\vec{\ell}$ towards direction $\vec{r}, \vartheta\left(\lambda, \vec{r}, \vec{r}^{\prime}\right)$ is the VSF, and $E(\lambda, \vec{r}, \vec{\ell})$ denotes the source radiance. By taking light polarization and multiple scattering into consideration, an analytic solution was developed in [85] by using the Stokes vector. Another analytical solution was devised in $[81,86]$ where the derivation was simplified by small angle approximation. Since it is very hard to find an exact analytical solution of RTE [84], numerical solutions of RTE have gained more attention compared to the solutions obtained by making assumptions and simplifications [87].

Monte-Carlo Methods. A Monte-Carlo simulation is a probabilistic numerical solver which mimics the underwater light propagation by emitting and tracking a large amount of photons [72]. A robust Monte-Carlo based model was designed in [88] by the U.S. Naval Research Laboratory. Recent research efforts on characterizing the UOWC channels by solving the RTE with Monte-Carlo simulations can be found in [89, 72, 90, 91].

\subsubsection{Oceanic Turbulence}

Oceanic turbulence is defined as the rapid variations in the refraction index due to fluctuations in the aquatic medium parameters such as pressure, density, salinity, temperature, etc. [50]. This phenomenon provokes inconstant light intensity reception that is referred to as scintillation yielding significant performance degradation.

Although UOWC channel modeling studies are mostly concentrated on obtaining a precise characterization of the absorption and scattering effects, the impact of oceanic turbulence on the system performance has not received the attention it deserves. As the physical mechanisms of atmospheric and oceanic turbulence share some similar features, several oceanic turbulence modeling studies employed traditional free-space optical (FSO) turbulence models. For example, the classical spectrum model of Kolmogorov was adopted for UOWC channels in [92]. Inspired by [92], a generic channel model was proposed in [93] by considering absorption, scattering, and turbulence. This model directly applies the well-known lognormal turbulence model, i.e.,

$$
f_{I}(I)=\frac{1}{I \sqrt{2 \pi \sigma}} \exp \left(-\frac{(\ln (I)-\mu)^{2}}{2 \sigma}\right),
$$

where $I$ is the received light intensity, $\mu$ is the mean logarithmic light intensity, and $\sigma$ is the scintillation index.

The impact of oceanic turbulence and depth on the underwater imaging were analyzed in [94, 95]. Adaptive optics were proposed in [96] to mitigate the negative effects of turbulence for UOWC and underwater imaging. In [97], the authors derived the power spectrum of refractive index fluctuations in turbulent sea water. The Gaussian light-beam propagation in turbulent sea water was studied in $[98,99,100]$. In the weak oceanic turbulence case, an aperture averaging method was analyzed and shown to improve the system performance by reducing the scintillation index [67]. In [101], the average speed of moving oceanic turbulence has been shown to have a major impact on the temporal correlation of the irradiance whereas the link distance has minor effects. Using the Rytov method, scintillation indices of different optical waves are evaluated in a turbulent aquatic medium [102].

\subsubsection{Pointing $\mathcal{E}$ Alignment}

Pointing and alignment are critical engineering tasks needed to maintain a constant reliable link between the optical transceivers. The pointing errors and misalignment are generally considered as a result of bore-sight and jitter [103] effects. The bore-sight is defined as a fixed displacement between the transmitter trajectory (i.e., beam center) and the center of the receiver aperture, which may be caused by the inaccurate receiver location information. On the other hand, the jitter is random dislocations between the light-beam and the aperture center due to the oceanic turbulence [67], depth-depended variations, deep currents [49], and random movements of the sea surface $[65,66]$. Even though the bore-sight can be mitigated by precise location information and effective PAT mechanisms, jitter is still a problem as the random nature of the oceanic environment cannot be controlled.

We should note that as the scattering effects become more significant (i.e., in coastal and turbid waters), tight pointing and alignment requirements are relaxed due to the high dispersion of the light-beam [104]. In [86], the authors investigated the impact of spatial spreading of an underwater light beam on the pointing accuracy. In [105], we investigate the end-to-end performance of multi-hop UOWCs in the presence of pointing mismatch due to the location uncertainty. Assuming that beamwidths can be manipulated by using adaptive op- 
tics, divergence angles are calculated based on estimation error of the underlying localization scheme. Numerical results show that all performance metrics are severely degraded in the absence of an effective PAT mechanism because of required high divergence angle to cover a large area for establishing a connected link. Additionally, broad beam/omnidirectional modems are developed to mitigate the PAT issues for UOWNs. For example, omnidirectional underwater optical base stations with multi-faceted spherical shape were proposed in [106, 107, 108]. Consequently, an omnidirectional optical transceiver was developed in [64] to mitigate the problem of pointing and tracking for UOWNs.

Neglecting the pointing errors caused by jitter, misalignment is modeled using the following beam spread function (BSF) [81, 109]:

$$
\begin{aligned}
& \operatorname{BSF}(\lambda, d, r)=P_{r}(\lambda, d) E(d, r)+\int_{0}^{\infty} P_{r}(\lambda, d) E(d, x) \\
& \times\left[\exp \left(\int_{0}^{d} b(\lambda) \tilde{\vartheta}(x(d-y)) d y\right)-1\right] J_{0}(y r) y d y,
\end{aligned}
$$

where $E(d, r)$ and $E(d, x)$ are the irradiance distributions of the laser source in spatial coordinates and in the spatial frequency domain, respectively; $d$ is the distance between transceivers; $r$ is the distance between the center points of aperture and the received light-beam; $\tilde{\vartheta}(\cdot)$ is the SPF. Using this model, the authors evaluated the BER performance of UOWC under misalignment conditions. In [104], the pointing error performance was investigated as a function of BSF under different water types. Effects of random movements of the sea surface on the jitter of transceivers were studied in [65] where the PDF of sea surface movements was considered as a two-dimensional Gaussian distribution. The impact of transmitter parameters such as divergence and elevation angles was also analyzed and simulated using Monte-Carlo method [66]. In [72], misalignment of point-to-point (P2P) communication was studied by using a Monte-Carlo simulation and verified with water tank experiments. The numerical results showed that given a sufficiently large transmission power, a small misalignment does not yield a significant performance loss for any water type [34].

\subsubsection{Multipath Fading and Delay Spread}

Due to the scattering and reflection effects, some portions of the emitted light-beam may follow different propagation paths with various traveling distances and may reach the receiver aperture at different time instants, yielding time dispersion (i.e., delay spread) and inter-symbol interference (ISI). Unlike the UAWC where the delay spread and ISI are quite considerable due to very long distances and low propagation velocities, these phenomena have not received much attention as a result of the high signal speed and the limited communication ranges of UOWC. Multipath fading can be more significant in shallow waters because of the reflections from the sea surface, the seabed, and the obstacles in the vicinity. The impact of spatial diversity on ISI was investigated in [110], where high data rates were observed to suffer more from ISI phenomenon. In order to quantify the time spread, the authors of [26] investigated the impact of the system design parameters such as the divergence angle and the receiver aperture size. Spatial and temporal dispersion of UOWC channel concerning pointing angle between the transmitter and the receiver was examined in [34]. Moreover, a theoretical model for beam spreading of UOWC channel was proposed in [81] which was validated through lab experiments. Also, the impact of spatial spreading of an underwater light beam on the pointing accuracy was investigated in [86]. Furthermore, in [111] experiments were presented to validate the Monte Carlo model for spatial and temporal dispersion of UOWC channel. The channel impulse response and time dispersion for UOWC channel in various types of water were studied in [89]. Monte Carlo based simulation model was proposed in [90] to study the underwater time domain pulse response for optical channel. In [85], through a time spread analysis it is deduced that ISI is significant at $50 \mathrm{~m}$ for a polarized light beam with $1 \mathrm{Gbps}$ data rate. However, a MonteCarlo simulation based channel characterization concludes that the time spread is negligible over short distances [72]. Recently, propagation behavior including the magnitude and phase of the UOWC was studied in [112] where the phase information provides extra knowledge about the forward scattered light. Most of these works show that dispersion is an issue for more turbid environments.

\subsection{Summary and Insights}

In order to motivate UOWCs, this section started with a comparison of virtues and drawbacks of the three main UWC systems: acoustic, RF, and optical. Moreover, it is pointed out that water types and depths have a major impact on the UOWC channel. Therefore, novel networking solutions accounting for these physical layer challenges are necessary to realize UOWNs. The research efforts on underwater optical wireless channel models including the Beer-Lambert law, radiative transfer theory, Monte-Carlo methods, the log-normal oceanic turbulence model, pointing errors and misalignment models were also presented. While Beer-Lambert law is an analytically simple method which merely focus on ballistic photons by assuming scattered photons are totally lost, radiative transfer theory is quite complicated because of the computational complexity in finding the exact analytical solution of the RTE. Therefore, there is a dire need for a realistic model with reasonable complexity. All these physical layer aspects can be regarded as the building blocks of UOWNs and play an important role to characterize the challenges and their potential solutions of the higher layers.

\section{Data Link Layer: Link Configurations and Multiple Ac- cess Schemes}

The data link layer is the protocol layer that conveys data between the neighbor network entities (i.e., single-hop or multihop connections) and, often provides functions to detect and correct possible physical layer errors. Regardless of the users ultimate destination, the data link layer undertakes the task of arbitrating among the users, who compete for the same network 
resources such as for time, frequency, space, and wavelength, in order to prevent frame collisions and it specifies protocols to detect and recover from such collisions. Even though the content of the first two subsections are not solely related to the data link layer, covering them in this section is especially important to provide valuable insights into the cross-layer optimization of the first two layers.

This section first discusses the requirement and design challenges for link layer, then compares wide-beam short-range and narrow-beam long-range transmission schemes and calls attention to the fundamental trade-off between the divergence angle (i.e., the coverage span) and the communication range (or the received power for a given range). Then, the power budget of the three main UOWC link configurations is presented, i.e., line of sight $(\mathrm{LoS})$, non line of sight $(\mathrm{NLoS})$, and retroreflective links. Following the error and data rate performance for UOWNs, potential multiple access schemes are also addressed.

\subsection{Requirements and Design Challenges}

In this section, we outline different requirements and design challenges for data link layer protocols of UOWNs.

Range-Beamwidth Tradeoff. It is of paramount importance to mitigate the short transmission range of UOWCs which delimits the overall network performance. Noting that increasing the transmission power is not a sustainable option, manipulating the range-beamwidth tradeoff and employing effective relaying techniques can help in extending the end-to-end reachability of UOWNs.

Coverage. The directivity of UOWCs yields a sector-shaped coverage region which posses unique challenges compared to the omnidirectional transmissions. The radius of this sector (i.e., transmission range) is inversely proportional to the central angle (i.e., divergence angle). A receiver within the coverage region of a transmitter is not necessarily able to receive signals since the receiver aperture must be pointing toward the light source, which hinders the connectivity of the network. Thus, an optimal coverage must be obtained via manipulating rangebeamwidth tradeoff for the maximum degree of connectivity.

Link Failures. Besides the UOWC channel impediments (i.e., absorption, scattering, turbulence, etc.), link failures are also easily affected by pointing and alignment disruptions caused by random movements of sea surface or deep currents. Faultprone links can be mitigated by operating at a larger beamwidth at the expense of a shorter transmission range. This approach can be further enhanced by considering opportunistic multicast link layer protocols to tackle the link failure problem.

PAT Mechanisms. As being closely related to the above discussions, precise PAT mechanisms are the most critical component of underwater transceivers to maximize the range, enhance the reliability, and provide an uninterrupted communication link. It is also necessary to employ accurate localization algorithms which have a direct impact on the precision of PAT mechanisms.
Energy Efficiency. Battery operated sensor nodes limit the network lifetime of UOWNs. Although energy harvesting is a promising method to develop energy self-sufficient UOWNs, the research on energy harvesting mechanisms for UOWNs is still not explored in-depth yet. Energy efficient packet forwarding protocols are also needed to improve the network lifetime of UOWNs.

\subsection{Narrow Beam vs. Wide Beam Light Sources}

Based on the divergence angle values, light sources can be classified into two broad categories: wide-beam and narrow beam sources, such as light emitting diodes (LEDs) and laser diodes, respectively. LEDs operate on the visible light communication (VLC) operates on LEDs combining the two main advantages: energy efficient indoor/outdoor illumination [113] and high-speed data delivery [114]. LEDs have already been commercialized by many startups, e.g., Light Fidelity (LiFi) [115]. Since VLC targets to serve multiple users concurrently, ongoing research efforts mostly concentrate on efficient resource sharing and multiple access schemes [116]. However, FSO communication focuses more on long-range and high data rate outdoor terrestrial optical wireless communication (TOWC) applications such as wireless X-hauling [117]. Unlike VLC, pointing, acquisitions, and tracking (PAT) functionality plays an important role for FSO communication systems to maintain a continuous system performance [118], thus, they can be employed in point-to-point (P2P) long-range outdoor links.

Atmospheric link losses are generally dominated by the beam spreading factor, $d^{-2}$, where $d$ is the communication distance. In the aquatic medium, however, the extinction loss, $e^{-c(\lambda) d}$, of nearly collimated light beams (e.g., lasers) dominates the beam spreading factor. On the other hand, the beam spreading factor $d^{-2}$ is the primary source of loss in the link budget calculations of light sources with broad divergence angles (e.g., LEDs). Hence, wide-beam light sources can communicate with nearby receivers scanned in a broad angle circular sector while narrow-beam light sources can reach distant receivers within a tight circular sector, as shown in Fig. 6a. In other words, there is a fundamental trade-off between the divergence angle (i.e., the spanned coverage area) and the transmission range (or received power for a fixed range). It must also be noted that even if the transmitter has a very tight divergence angle, the receivers can observe a slightly diffused light beam because of the aquatic medium, which is more significant in water types with a severe scattering nature, e.g., in turbid waters.

Accordingly, the narrow-beam light sources have the following advantages [119]: (i) higher power reception and longer communication ranges; (ii) reduced time spread due to the relatively high ratio of "ballistic" photons which propagates without scattering. Monte Carlo simulations show that $90 \%$ of photons arrive within $10 \mathrm{~ns}$ and $2 \mathrm{~ns}$ for the wide-beam and the narrow-beam transmissions, respectively. The arrival time can even be reduced to $90 \mathrm{ps}$ if the narrow-beam transmission is received by a receiver with $0.1 \mathrm{mrad}$ field of view (FoV); and (iii) improved spectral and spatial filtering options are available since the receiver FoV can be reduced significantly due to the 


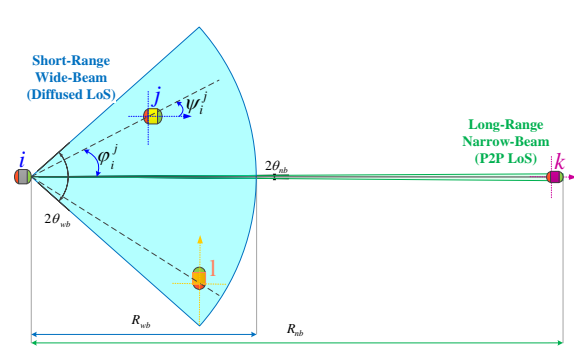

(a) LOS

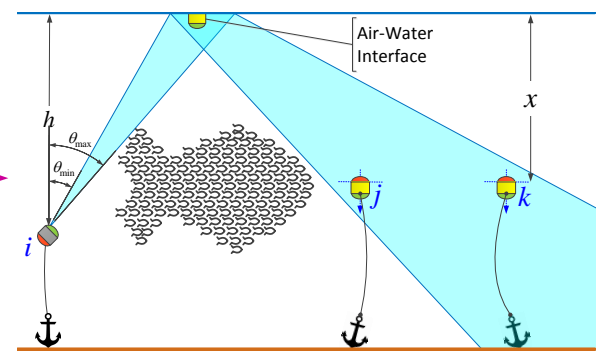

(b) NLoS (Reflective)

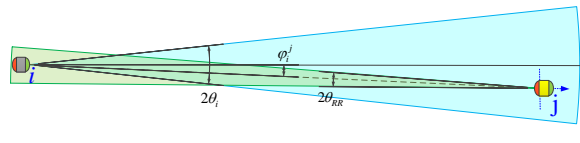

(c) Retro Reflective

Figure 6: Underwater optical link configurations: a) LoS, b) NLoS (Reflective), and c) Retro Reflective.

limited light diffusion at the receiver. Albeit these advantages, narrow-beam transmission requires accurate PAT mechanisms which are addressed in Section 4.2. Fletcher et. al. considers a narrow-beam laser communication system over 20 extinction length (around $132 \mathrm{~m}$ for an extinction length of $6.6 \mathrm{~m}$ ) with $100 \mathrm{~mW}$ transmission and $2 \mathrm{~cm}$ aperture size [119]. 16-ary pulse position modulation with 1/2-rate forward error correction (FEC) achieves 1 Gbps capacity at a wavelength of $515 \mathrm{~nm}$ where the attenuation loss is $87 \mathrm{~dB}$ and noiseless sensitivity is $2.9 \mathrm{~b} /$ photon. For comparison purposes, the same set up was also considered for a wide-beam transmitter which achieves only $3.5 \mathrm{kbps}$.

\subsection{Aquatic Optical Link Configurations}

In this section, we consider three main link configurations for UOWNs: 1) LoS Links, 2) NLoS Links, and 3) Retro-Reflective Links.

\subsubsection{LoS Links}

LoS communication is the most straightforward form of optical links where transceivers communicate over an unobscured link which can either happen in a diffused or a $\mathrm{P} 2 \mathrm{P}$ fashion as illustrated in Fig. 6a. Even implementing the P2P LoS links for stationary transceivers is a trivial task in clear ocean, it may require sophisticated PAT mechanisms to keep transceivers boresighted in the case of mobility.

For a generic optical transmitter node $i$ and receiver node $j$, propagation loss factor is given based on Beer Lambert's Law as [120]

$$
L_{i j}\left(\lambda, d_{i j}\right)=\exp \left\{-c(\lambda) d_{i j}\right\},
$$

where $d_{i j}$ is the Euclidian distance between the transceivers and $\varphi_{i}^{j}$ is the angle between the receiver plane and the transmitter trajectory. Likewise, geometric gain (a.k.a. telescope gain) of the LoS link is given as [27]

$$
G_{i j}^{L o S}=\left\{\begin{array}{ll}
\frac{A_{j}}{d_{i j}^{2}} \frac{\cos \left(\varphi_{i}^{j}\right)}{2 \pi\left[1-\cos \left(\theta_{i}\right)\right]},-\pi / 2 \leq \varphi_{i}^{j} \leq \pi / 2 \\
0, \quad \text { otherwise }
\end{array},\right.
$$

where $A_{j}$ is the receiver aperture area of node $j$ and $\theta_{i}$ is the beam divergence angle of transmitter node $i$. In order to concentrate the transmitted energy on receiver aperture, the divergence angle of laser-diodes are generally designed to be a few milliradians or less [121] whereas typical LEDs can have divergence angles less than 140 milliradians to diffuse light to wide angles [122]. Accordingly, received power can be formulated as a product of transmission power, transceivers' efficiency, telescope gain, and path loss factor, i.e.,

$$
P_{r}^{j}=P_{t}^{i} \eta_{t}^{i} \eta_{j}^{r} G_{i j}^{L o S} \chi\left(\psi_{i}^{j}\right) L_{i j}\left(c(\lambda), \frac{d_{i j}}{\cos \left(\varphi_{i}^{j}\right)}\right),
$$

where $P_{t}^{i}$ is the transmission power, $\eta_{t}^{i}$ and $\eta_{j}^{r}$ are transmitter and receiver efficiency, respectively; $\chi\left(\psi_{i}^{j}\right)$ is the concentrator gain [123], which is defined for non-imaging concentrators as [124]

$$
\chi\left(\psi_{i}^{j}\right)=\left\{\begin{array}{l}
\frac{n^{2}}{\sin ^{2}\left(\Psi_{j}\right)}, 0 \leq \psi_{i}^{j} \leq \Psi_{j} \\
0, \quad \psi_{i}^{j}>\Psi_{j}
\end{array},\right.
$$

$\psi_{i}^{j}$ is the angle of incidence w.r.t. the receiver axis, $\Psi_{j}$ is the concentrator FoV which can be $\pi / 2$ and down to $\pi / 6$ for the hemisphere and parabolic concentrators, respectively; and $n$ is the internal refractive index. Notice that the receiver gain increases as the FoV decreases. Hemispherical lens are common nonimaging concentrators [125] which can achieve $\Psi_{j} \approx \pi / 2$ and $\chi\left(\varphi_{i}^{j}\right) \approx n^{2}$ over its entire FoV. The compound parabolic concentrator [124] is another type of nonimaging concentrators and can obtain a much higher gain in return for a narrower FoV, which is especially more desirable for P2P-LoS links. Since it is easy to implement, most of the experimental studies considered LoS links under different water characteristics and modulation schemes using a variety of transmitter hardware [126, 127, 107, 37, 26, 128, 129]. However, the link budget model in (9) is based on geometric loss only and does not consider the scattering effect. Hence, Elamassie et. al. recently modified this model to take both geometrical and scattering loss into account in $[130,131]$ where an additional parameter is introduced to involve scattered photons in the received signal power. The values of this parameter are validated by Monte Carlo simulations under different water types and hardware parameters.

\subsubsection{NLoS Links}

LoS links may not always be available due to the obstructions within the underwater topology, PAT errors, mobility, and random orientations of the transceivers, etc. In such cases, a diffused light beam which is reflected over sea surface (or alternatively a mirror located in an appropriate location) can be beneficial to facilitate a point-to-multipoint (P2M) (a.k.a. mul- 


$$
G_{i j}^{N L o S}= \begin{cases}\frac{A_{j} \cos \left(\varphi_{i}^{j}\right)}{2 A_{a n n}}\left(\left[\frac{\tan \left(\theta_{t}-\varphi_{i}^{j}\right)}{\tan \left(\theta_{t}+\varphi_{i}^{j}\right)}\right]^{2}\left[\frac{\sin \left(\theta_{t}-\varphi_{i}^{j}\right)}{\sin \left(\theta_{t}+\varphi_{i}^{j}\right)}\right]^{2}\right) & , \theta_{\min } \leq \varphi_{i}^{j} \leq \theta_{c} \\ \frac{A_{j} \cos \left(\varphi_{i}^{j}\right)}{2 A_{a n n}} & , \theta_{c} \leq \varphi_{i}^{j} \leq \theta_{\max } \\ 0 & , \text { otherwise }\end{cases}
$$

ticasting) transmission to reach obscured receivers, as depicted in Fig. 6b. Assuming that the transceivers are oriented vertically upward, the transmitted light beam is characterized by inner and outer angles $\theta_{\min }$ and $\theta_{\max }$, respectively. As per the Fresnel's law, propagating light is partially refracted and partially reflected at interfaces between the media with different refractive indices. Therefore, the light beam transmitted from depth $h$ is partially reflected from the sea surface and illuminates an annular surface $A_{a n n}$ at depth $x$ with equal power density. $A_{\text {ann }}$ is given by

$$
A_{\text {ann }}=2 \pi(h+x)^{2}\left[\cos \left(\theta_{\min }\right)-\cos \left(\theta_{\max }\right)\right],
$$

which defines an annular area taken from a sphere of radius $h+x$ [120]. Assuming that sea surface is modeled as smooth (i.e., incident angle is equal to the perpendicular angle between the receiver plane and the transmitter-receiver trajectory, i.e., $\varphi_{i}^{j}$ ), the telescope gain of the NLoS links is given in (11) where $\theta_{t}$ is the angle of transmission, $\theta_{c} \triangleq \sin ^{-1}\left(\frac{n_{A}}{n_{W}}\right)$ is the critical angle (i.e., the angle of incidence above that the total internal reflection occurs), $n_{A}$ is the refraction index of air, and $n_{W}$ is the refraction index of water. Accordingly, received power at node $j$ is expressed as follows

$$
P_{r}^{j}=P_{t}^{i} \eta_{t}^{i} \eta_{j}^{r} G_{i j}^{N L o S} \chi\left(\psi_{i}^{j}\right) L_{i j}\left(c(\lambda), \frac{h+x}{\cos \left(\varphi_{i}^{j}\right)}\right) .
$$

LoS and NLoS links have been compared by Jasman et. al. in [132] where they have demonstrated that $100 \mathrm{MHz}$ bandwidth availability of LoS links is reduced to $20 \mathrm{MHz}$ in case of NLoS even in clear water conditions. Indeed, such a reduction is not a surprise due to the reflection losses at the sea surface and diffusion of the reflected light beam. Furthermore, multi-scattering effect of NLoS links was addressed in [133] and [134]. As shown in Fig. 6b, reflective communications can also be used for establishing links between underwater nodes and air-water interface buoys that relays received optical signals to terrestrial destinations (e.g., mobile, onshore, or airborne stations) via TOWCs or RF communications.

\subsubsection{Retro-Reflective Links}

Similar to backscatter communication in RF systems, retroreflective communication consists of a light source and a reflector. While the light source could be a sophisticated system with high transmission power, the reflector behaves as an interrogator as it lacks the ability to fulfill transceiver operations due to its simple architecture with low power availability. Therefore, the continuous light beam emitted from the source is modulated and reflected back to the receiver. Retro-reflective communications can be considered in two cases [48]: photon limited case

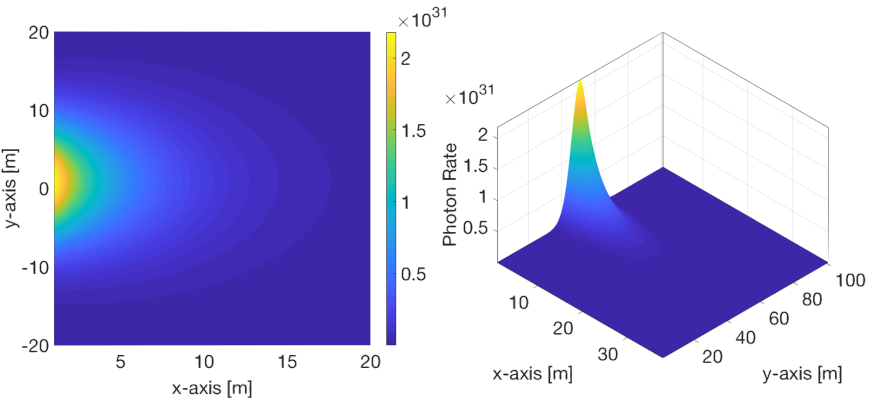

Figure 7: Demonstration of photon arrival rate for LoS link [137].

and contrast limited case which take place in clear and turbid water, respectively. In the former case, absorption is the dominant effect which reduces the number of photons received by the reflector. Furthermore, the accuracy of PAT mechanisms at both sides plays a significant role in receiving enough informationbearing photons. In the latter case, scattering is the dominant factor which mainly determines retro-reflective link range and capacity. Contrast limitation is especially important for underwater imaging applications as a reduction in photon quantity directly reduces the image contrast, which can be considerably improved by exploiting polarization discrimination [135, 136]. If the receiver has enough power resource, the reflector can even amplify the modulated light beam in order to achieve a better performance both in photon and contrast limited scenarios [137].

Based on the geometric gain of LoS links in (8), telescope gain of the retro-reflective links is expressed as [27]

$$
G_{j i}^{R R}=\left\{\begin{array}{l}
\frac{A_{j}}{d_{i j}^{2}} \frac{\cos \left(\varphi_{i}^{j}\right)}{2 \pi\left[1-\cos \left(\theta_{i}\right)\right]} \frac{A_{R R} \cos \left(\varphi_{j}^{i}\right)}{\pi\left[d_{i j} \tan \left(\theta_{R R}\right)\right]^{2}},-\pi / 2 \leq \varphi_{j}^{i} \leq \pi / 2 \\
0, \quad \text { otherwise }
\end{array}\right.
$$

where $A_{R R}$ is the aperture area of the reflector, $\theta_{R R}$ is the divergence angle of the reflector, and $\varphi_{i}^{j}$ is the angle between receiver trajectory of the source and the reflector trajectory. Accordingly, reflected light beam is received back by the source node $i$ as follows

$$
P_{r}^{i}=P_{t}^{i} \eta_{t}^{i} \eta_{i}^{r} \eta_{j}^{R R} G_{i j}^{R R} \chi\left(\psi_{j}^{i}\right) L_{i j}\left(c(\lambda), \frac{2 d_{i j}}{\cos \left(\varphi_{i}^{j}\right)}\right),
$$

where $\eta_{j}^{R R}$ is the retro-reflector efficiency. It is worthy to note here that the expressions in (14) and (15) only consider the geometric losses whereas the expressions for scatter-limited case can be found in [136] 


\subsubsection{Comparison of Link Configurations}

For a better connectivity, UOWNs should be able to employ different link configurations according to network conditions. For instance, LoS links should always be preferred as long as the network deployment area is free of obstacles. However, establishing LoS links are not always possible because of obstructions imposed by ocean surface topography or marine life activity. In that case, network deployment and link configuration strategy may alleviate such cases by employing NLoS links to keep network connected. One should note that NLoS provide much more low rates compared to LoS counter parts as a result of increased propagation distance and reflection losses at the sea surface or the installed mirrors. Finally, retro-reflective links are quite interesting to be employed at sink nodes with power availability such as surface station or boats. In such a case, it is possible to deploy nodes with limited energy sources and keep them always connected via adaptive transmission schemes at the retro-reflection sources. Again, retro-reflection suffers from doubled traveling distance and losses during the reflection and back-scatter modulation stages.

\subsection{Error and Data Rate Performance}

Before proceeding to the medium access schemes, it is important to quantify the error and data rate performance of these link configurations based on a common and straightforward detection technique. Indeed, expressions for performance metrics take different based on the underlying system configurations, hardware parameters, and modulation techniques. However, the signal-to-noise ratio, that can be obtained by using link budget expressions in previous subsections, can be regarded as the main variable that ties underwater environment with wellknown modulation schemes. By referring readers who are interested in performance analysis of various systems to existing surveys in [48, 23], we content ourselves with intensity modulation direct detection (IM/DD) on-off keying (OOK) with silicon photo-multipliers based photon counter detectors [138]. Photon arrivals are generally assumed to be a Poisson distributed function, therefore, the photon arrival rate within a slot duration $T$ is given by

$$
p_{i}^{j}=\frac{P_{j}^{r} \eta_{D}^{j}}{T R_{i}^{j} \hbar c},
$$

where $P_{j}^{r}$ is the received power, $\eta_{D}^{j}$ is the detector efficiency, $R_{i}^{j}$ is the data rate, $\hbar$ is the Plank constant, and $c$ is the speed of light. Fig. 7 shows the photon arrival rate w.r.t. xy-coordinates for a sensor fixed at origin and pointing in positive $\mathrm{x}$-axis direction while the receiver is located at different location and its receiver directed to the origin. Assuming a large number of photon reception, then according to the central limit theorem, Poisson distributed photon arrivals can be approximated by a Gaussian distribution and the bit error rate (BER) is given by

$$
B E R_{i}^{j}=\frac{1}{2} \operatorname{erfc}\left[\sqrt{\frac{T}{2}}\left(\sqrt{p_{i j}^{1}}-\sqrt{p_{i j}^{0}}\right)\right],
$$

where $\operatorname{erfc}(\cdot)$ is the complementary error function, $p_{i, j}^{0}=p_{b g}+$ $p_{d c}$ and $p_{i, j}^{1}=p_{i}^{j}+p_{i, j}^{0}$ are the photon arrival rates when binary 1 and binary 0 are transmitted, respectively; $p_{b g}$ and $p_{d c}$ are the background illumination noise and additive noise due to dark counts, respectively. For a given BER $\overline{B E R}_{i}^{j}$, achievable data rate can be obtained from (17) as

$$
R_{i}^{j}=\frac{P_{i}^{j} \eta_{D}^{j} \lambda}{T \hbar c\left[\left(\operatorname{erfc}^{-1}\left(2 \overline{B E R_{i}^{j}}\right) \sqrt{\frac{2}{T}}+\sqrt{p_{i j}^{0}}\right)^{2}-p_{i j}^{0}\right]} .
$$

Since hard decision forward error correction (HD-FEC) can successfully identify and correct all bit errors below an FECBER threshold, one can set $\overline{B E R}_{i}^{j} \leq 3.8 \times 10^{-4}$ as recommended by the International Telecommunication Union Standardization Sector (ITU-T) [139].

\subsection{Multiple Access Schemes}

For infrastructure based UOWNs, many researchers conceptualized omnidirectional OBSs by designing them in multi-faceted spherical shape which has single or multiple transceivers at each face [106, 107, 140, 108]. Therefore, underwater OBSs can be designed as geodesic polyhedra as shown in Fig. 8 along with its implementation [106, 107]. Geodesic polyhedra approximates spheres with triangles and can be a good solution against underwater pressure as the geodesic domes are known to withstand heavy structure loads by distributing the structural stress over its rigid triangular building blocks [141]. Notice that as the number of faces (pentagonal/hexagonal shapes in Fig. 8) increases, it is possible to employ narrower transmitter divergence and receiver FoV angles which naturally yields longer transmission range and higher receiver gain [c.f. Fig. 8], respectively. In addition to their spatial reuse and angular diversity advantages [140], OBSs can also provide flexibility as each LED on a face can be exploited to serve for fulfilling a specific task.

In OBS based cellular UOWNs, there exists two main interference scenarios: intercell interference (ICI) and intracell interference which is also referred to as multiple access interference (MAI). While the former happens when a user receives signals from other users using the same network resources within the adjacent cells, the latter occurs when a user observes interfering signals from users sharing the same cell resources. Compared to VLC systems, intercell interference expected to be at low levels due to the severe aquatic channel impairments and can even be further reduced by intelligent OBS deployment strategies. Nonetheless, intracell interference still stays as a first and foremost research challenge for both downlink (DL) and uplink (UL) transmission. Hence, in addition to efficient resource allocation strategies, OBSs necessitate multicarrier transmission schemes and multiple access protocols to serve several users simultaneously.

Multiple access schemes can be categorized into electrical and optical multiplexing subcategories. Electrical multiplexing schemes consist of time division multiple access 


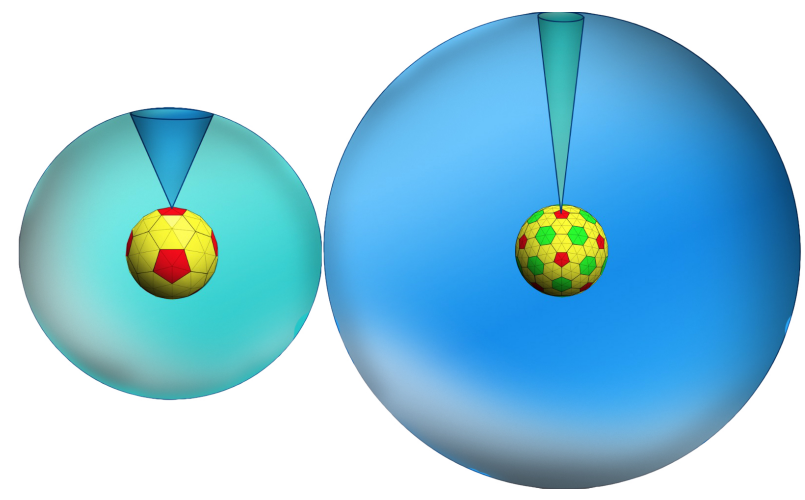

Figure 8: Illustration of omni-directional transceivers.

(TDMA), frequency-division multiple access (FDMA), codedivision multiple access (CDMA), and non-orthogonal multiple access (NOMA) whereas optical multiplexing schemes contain wavelength-division multiple access (WDMA) and spacedivision multiple access (SDMA). To the best of authors' knowledge, there is no research efforts on UOWN multiple access schemes excluding the optical CDMA [142, 143, 144]. In the remainder, we present multicarrier transmission techniques along with corresponding multiple access schemes for UOWNs, which is followed by comparison of multiplexing schemes in Table 4.

\subsubsection{Time Division Multiple Access}

TDMA is a synchronous channel access scheme where nonoverlapping time slots are assigned to different users as per the requested quality of service (QoS) levels. Hence, TDMA does not allow nodes to transmit simultaneously and independently. In UAWC systems, TDMA provides a limited bandwidth efficiency because low propagation speed requires long time guards to prevent packet collisions of the adjacent time slots [54], which may not be the case for UOWC systems thanks to low propagation delays. TDMA can support high energy efficiency in return for reduced capacity per user [123]. Nevertheless, TDMA requires efficient scheduling techniques in order to overcome the MAI. A potential scheduling scheme could be based on users rather than LEDs embedded on OBSs as they can be much larger than the number of users. Even though TDMA has not attracted the attention for UOWNs yet, it can be motivated by research efforts on TDMA based VLC systems: As a potential solution, each LED is orthogonally allocated to a time slot in [145] and a block encoding TDM is exploited in [146] where one LED from each LED group is allowed to transmit. In [147], TDMA is considered for UL transmission where each user has certain time slots to transmit such that identity of the transmitting users can be recognized as per the scheduling policy.

\subsubsection{Frequency Division Multiple Access}

FDMA scheme permits multiple users to transmit momentarily over non-overlapping frequencies/subcarriers within a cell area. In FDMA, the intensity of the signals is modulated over the non-overlapping frequencies. Noting that FDMA is not suit- able for acoustic systems due to the limited bandwidth availability [56], it offers high spectral efficiency and robustness against intersymbol interference (ISI) [148] for optical wireless communications. However, it lacks energy efficiency which deteriorates with the increasing number of subcarriers [149]. Orthogonal FDMA (OFDMA) and interleaved FDMA (IFDMA) are two well-known schemes studied extensively for OWCs due to their spectral efficeiny and robustness to ISI [116].

OFDMA allocates each user with several time slots and frequency blocks which spans a number of orthogonal frequency division multiplexing (OFDM) subcarriers. Because of the real and unipolar valued signal requirements of the IM, conventional OFDM cannot be directly applied to optical OFDM (OOFDM) systems. In return for losing half of the bandwidth, reality constraint can be satisfied by applying Hermitian symmetry on inverse fast Fourier transform inputs. Positivity of the signals can be achieved either by direct current biased optical OFDM (DCO-OFDM) [150] or asymmetrically clipped optical OFDM (ACO-OFDM) [90]. The former adds a DC bias before transmission which may cause overheating and high signal distortion. At the expense of BER performance degradation and increased complexity [151], several peak-to-average power ratio (PAPR) reduction techniques were proposed to overcome these problems $[152,153]$. In order to obtain unipolarity, ACOOFDM technique clips the signal at zero level [154] and transmits only the positive part of the signal. Even if it is more energy efficient than the DCO-OFDM, bandwidth utilization is quite low because of using only half of the subcarriers for data transmission.

Optical OFDMA (O-OFDMA) was proposed in [155] which has a lower decoding complexity and power efficiency in comparison with O-OFDM based interleave division multiple access. In O-OFDMA only a portion of the total sub-carriers transmit non-zero data because each sub-carrier is allocated to a single user. Also, in O-OFDMA, the data rate is changed with the modulation size $M$, since it is based on $M$-ary modulation [155]. Nevertheless, in [156], the authors have considered two handover schemes for users within the intersection area of two optical transmitters. In the first scheme, the user combines the signal of both transmitters, while in the second scheme each transmitter uses a dedicated band for the user. IFDMA was proposed in [157] to mitigate the high PAPR effects of O-OFDMA where it was shown that IFDMA have lower computational complexity than O-OFDMA and it reduces the synchronization errors.

\subsubsection{Code Division Multiple Access}

Optical code division multiplexing (OCDM) is a multiplexing scheme where communication channels are distinguished by optical orthogonal codes in addition to time and wavelength [158, 159]. As shown in Fig. 9, the data stream is multiplied by a code sequence either in the time domain, wavelength domain, or even as a combination of both (i.e., 2D coding) [160]. In the time domain, a bit duration is divided into smaller time slots which are called chips. Bipolar time-encoding is a coherent technique that manipulates the phase of the optical signal and needs phase accuracy. As an alternative, positive time encoding 

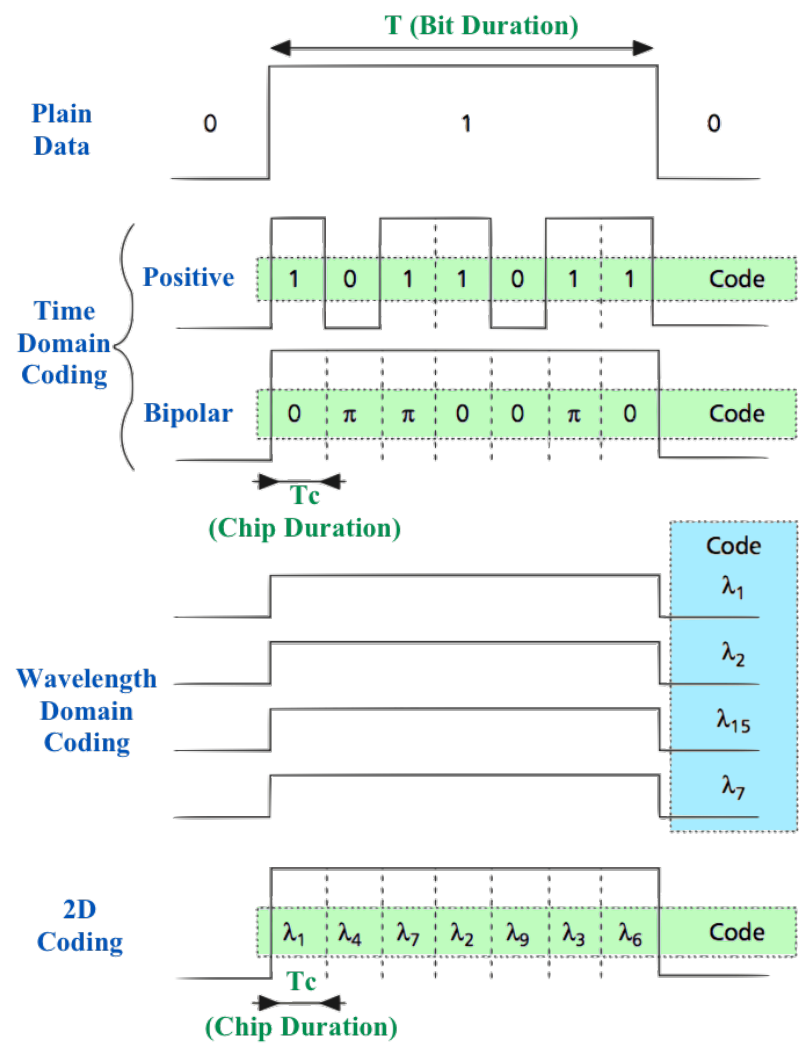

Figure 9: Illustration of OCDMA dimensions.

is non-coherent which manipulates the power of the optical signal without requiring any phase information [161]. On the other hand, a wavelength-encoded signal consists of a unique subset of wavelengths in order to form the code. Finally, 2D coding combines both time spreading and wavelength assignment such that a data stream is constituted as successive chips of different wavelengths. In the receiver side, decoding is performed by applying the reverse operations of the encoding. Accordingly, optical CDMA (OCDMA) employs OCDM technique to mediate multiple asynchronous nodes in sharing common network resources. Thanks to its high spectral efficiency, distributive, and asynchronous nature; OCDMA has received much attention to be employed in UOWNs [142, 143, 144]. In [142], the authors have addressed the structures, principles, and performance analysis of OCDMA based cellular UOWNs where OBSs are connected to a central optical network controller. In [143], the performance of relay-assisted OCDMA networks was characterized by the turbulent channels. Finally, potential and challenges (e.g., mobility, cell edge coverage, blockage avoidance, power control, etc.) of OCDMA networks were presented in [144].

\subsubsection{Non-Orthogonal Multiple Access}

NOMA is also referred to as power domain multiple access where user signals are superposed in a way that each signal is allocated to a distinct power level depending upon the channel conditions. While NOMA allocates more power to users with bad channel conditions compared to those with good channel condition. Employing successive interference cancellation, the user allocated with high powers can cancel the interference of the users with the low power allocation. Thus, all users can occupy the available entire time-frequency resources and increase overall system performance significantly [116]. Even though NOMA has attracted attention for VLC systems [162, 163], there is no study targeting NOMA for UOWCs.

\subsubsection{Wavelength Division Multiple Access}

WDMA facilitate the multi-user access harnessing the wavelength division multiplexing (WDM) such that each user has a dedicated wavelength along with an optical tunable reception filter in order to operate on assigned wavelength. WDM multiplexes a number of optical signals at different wavelengths (i.e., color) into a single one. Coarse and dense WDM are two standard types which are named based on the available number of channels and their spacing. Even if WDMA reduces the signal processing complexity to a great extent, it may significantly increase the hardware complexity and cost [164]. Since underwater operational wavelength is different from TOWCs, it is necessary to standardize the WDM channels and their spacing for blue-green wavelengths. Standardization efforts should particularly consider available narrow window of minimal absorption of the potential wavelengths. It is also important to develop efficient wavelength assignment policy as the nodes in UOWNs can observe different channel conditions at different wavelengths because of varying water types and depths.

\subsubsection{Space Division Multiple Access}

SDMA harnesses the spatial distribution of the users and directivity of the light beam propagation to permit parallel transmission on the same network resources which can either be in time, frequency/wavelength, or code domains. In [165], random grouping and optimal grouping approaches were proposed for an SDMA based VLC system and obtained results have shown that SDMA can offer 10 times higher throughput than the conventional TDMA scheme. Similarly a $2 \times 2$ MIMObased SDMA approach is used in [166] for UOWC with two orthogonal linear polarized laser beams to provide high speed underwater communications. Notice that SDMA is a potential technique to be employed for underwater OBSs as they can benefit from both spatial and angular diversity.

Another potential idea to increase the capacity of UOWC links is to use the space division multiplexing (SDM). SDM was suggested as a potential solution to cope with the upcoming capacity crunch in optical fiber networks and free space optics [167]. The core idea behind SDM is to use the spatial structure of the light as an additional degree of freedom for optical communications. Each spatial mode in SDM based communication systems can be viewed as an independent data carrier increasing the transmission throughput. A particular approach to encode information underwater is to use orbital angular momentum modes (OAM). OAM modes are Laguerre Gaussian modes with a null radial index and have been used lately as information carriers [168]. The orbital component of optical angular momentum $\ell$, not associated with the polarization of an optical field but rather with its wave-front, was discovered in 1992 by Allen et al. in [169]. In simple words, in an OAM 
Table 4: Comparison of multiplexing schemes

\begin{tabular}{|c|c|c|c|}
\hline Type & Domain & Scheme & References \\
\hline \multirow{4}{*}{ 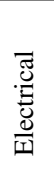 } & Time & TDMA & {$[54,123,145,146,147]$} \\
\hline & Frequency & FDMA & $\begin{array}{l}\text { DCO-OFDM [150, 151, 152, 153], } \\
\text { ACO-OFDM [90, 154], O-OFDMA } \\
{[155,156], \text { I-FDMA [157] }}\end{array}$ \\
\hline & Code & OCDMA & {$[142,143,144,158,159,160,161]$} \\
\hline & Power & NOMA & {$[162,163]$} \\
\hline \multirow[b]{2}{*}{ 苞 } & Wavelength & WDMA & [164] \\
\hline & Space/Mode & SDMA & $\begin{array}{l}\text { SDMA [165], SDM [167], OAM } \\
{[168,169,170,171,173,174,166 \text {, }} \\
175,176]\end{array}$ \\
\hline
\end{tabular}

mode, light is twisted like a corkscrew around its axis of propagation. It has been demonstrated that OAM beams maintain their azimuthal indices (twist configurations) as they propagate through turbulent water [170]. The first demonstration of an OAM-based underwater communication was reported by Baghdady et al. in [171] using blue OAM beams achieving an aggregated transmission capacity of 3Gbit/s over a distance of 2.94 $\mathrm{m}$. Authors of [172] performed lab experiments and reported a $1.2 \mathrm{~m}$ transmission distance with aggregated data-rate of 40 Gbit/s, using 4 OAM modes generated using green lasers. A one-to-many multicasting communication with 4 OAM carrying 8QAM-OFDM signals was demonstrated in [173]. Nonline-of-sight (NLoS) underwater communication using OAM was further demonstrated. The authors used the water-air interface as a mirror, through the total internal reflection principle, to establish a communication link between two terminal with no direct LoS path (blocked path) [174]. A future research direction to explore to further increase the capacity of UWOC systems is to use modes of light from the full Laguerre Gaussian mode basis (i.e., OAM modes with non-radial indices) as demonstrated in free space [175]. The potential, challenges, and perspectives of UWOC using OAMs are discussed in [176].

\subsection{Summary and Insights}

Data link layer is the protocol layer that handles data transfer among the neighbor network entities and provides functions to detect and correct physical layer errors, arbitrates among the users to prevent frame collisions and specify protocols to detect and recover from such collisions. This section first calls attention to the fundamental tradeoff between the divergence angle and communication range by comparing wide-beam shortrange and narrow-beam long-range transmission schemes. In the upcoming sections, we frequently refer to this tradeoff as it has a significant impact on the higher layer problems and their potential solutions. According to the Beer-Lambert Law based channel model, the power budget of three main UOWC link configurations is presented: line of sight $(\mathrm{LoS})$, non-line of sight (NLoS), and retro-reflective links. Power reception, bit error rates, and achievable data rates are quantified as a function of antenna gains, extinction coefficients, distance and geometric losses, and hardware parameters. After typifying two main interference scenarios, i.e. MAI and ICI, potential multiple access solutions are addressed including TDMA, FDMA, CDMA, NOMA, WDMA, and SDMA. Noting that none of them is the best option in all cases, a proper multiple access scheme must be determined based on operator needs. Moreover, OBS concept is introduced to overcome directivity limitation of UOWCs which can pave the way for benefiting from the advantages of cellular UOWNs.

\section{Network Layer: Relaying Techniques and Routing Pro- tocols}

Due to the communication range limitations of UOWCs, relay-assisted UOWC is a key enabler technique to realize UOWNs by expanding coverage area, extending the communication range, enhancing energy efficiency, providing cooperative diversity, and improving the end-to-end system performance [177]. However, the full benefit of relay-assisted UOWCs can be obtained by effective routing algorithms taking the underwater propagation characteristics of light beams into account.

Therefore, this section first discusses the requirement and design challenges for network layer, then covers serial relaying and parallel relaying techniques using decode-and-forward (DF), amplify-and-forward (AF) methods, and Bit-detectand-forward (BDF). After that, various routing protocols for UOWNs are surveyed. Moreover, potential routing protocols from underwater acoustic networks are studied which can be implemented for UOWNs. These potential routing protocols include location-based routing, source-based routing, hop-byhop routing, cross-layer routing, clustered routing, reinforcement learning, and energy-time efficient routing. Fig. 10 and Fig. 12 classify relaying and routing techniques surveyed in this paper, respectively.

\subsection{Requirements and Design Challenges}

In addition to the data link layer issues, the routing protocols must account for the following requirements and design challenges which mainly determine the end-to-end performance:

Route Discovery. Hop count is one of the primary metrics used to determine the route between the source node and the target node. However, application requirements and the current state of the network may require to account for other prominent parameters including but not limited to BER, achievable data rate, latency, and complexity. Noting that each metric has unique design considerations, novel routing protocols need to be devised for UOWNs as per the demands of underlying applications.

Network Lifetime. Prolonging the network lifetime is a critical objective especially for long-term monitoring of an underwater environment. However, deployed sensor nodes are typically battery powered which requires to plan routes with the minimum energy cost to improve the network lifetime. Existing energy efficient routing protocols (e.g., [178]) can be adapted to UOWNs by considering the unique features of UOWC channels. 
Localization Integrity. Since location information is a prerequisite to establish direct links between the nodes, geographic routing protocols are natural candidates for UOWNs. However, unavailability of GPS signals in the aquatic medium necessitates developing novel localization algorithms. Even though several localization methods are proposed for UOWNs [c.f. Section VIII], integration of localization and routing algorithms is a must to evaluate the impacts of accuracy on the link quality and route discovery.

Connectivity and Adaptability. Connectivity is of vital importance for both networking and localization aspects. The sparsity of the network can cause disconnectivity which yields a partitioned network where some of the nodes are not reachable at all albeit the existence of a perfect routing protocol. Moreover, the degree of connectivity has a reciprocal relationship with the localization. While a well-connected network improves the localization accuracy, a more accurate localization yields more efficient routing and reliable links via more precise PAT mechanisms, which eventually increases the connectivity. By considering the impacts of node failures, mobility, and dynamics of underwater environments on the adjacency matrix of UOWNs, the routing protocol should also be aware of dynamically changing connectivity and adapt itself by drawing alternative paths.

Cost and Complexity. Routing protocols can be implemented either in a centralized or distributed fashion. On the one hand, centralized protocols are suitable for end-to-end performance provisioning. However, its computational cost and communication overhead results in high power consumption due to the need for the entire network state information. On the other hand, distributed protocols allow nodes to operate based on their local information which requires less message passing and power expenditure. It is worth noting that in comparison with the centralized solutions, distributed protocols can deliver poor performance in sparse networks due to the limited connectivity. Fortunately, this limitation of the distributed solutions can be alleviated by parallel relaying techniques.

Latency. Real-time applications of UOWNs require ultrareliable and low-latency end-to-end performance which are often contradictory objectives. Low-latency requires high data rates to minimize the packet transmission time and less number of hops to reduce the processing and coordination delay at each relay. However, packet delivery ratio decreases with the increasing data rates, that harms the reliability. Therefore, there is a dire need to develop routing protocols which reap the full benefit of high data rates and propagation speed of UOWCs while ensuring the ultra-reliability and low-latency.

\subsection{Relaying Techniques}

As depicted in Fig 11, relaying can be implemented by involving either a single node or multiple nodes at each hop, which are referred to as serial and parallel transmission, respectively.

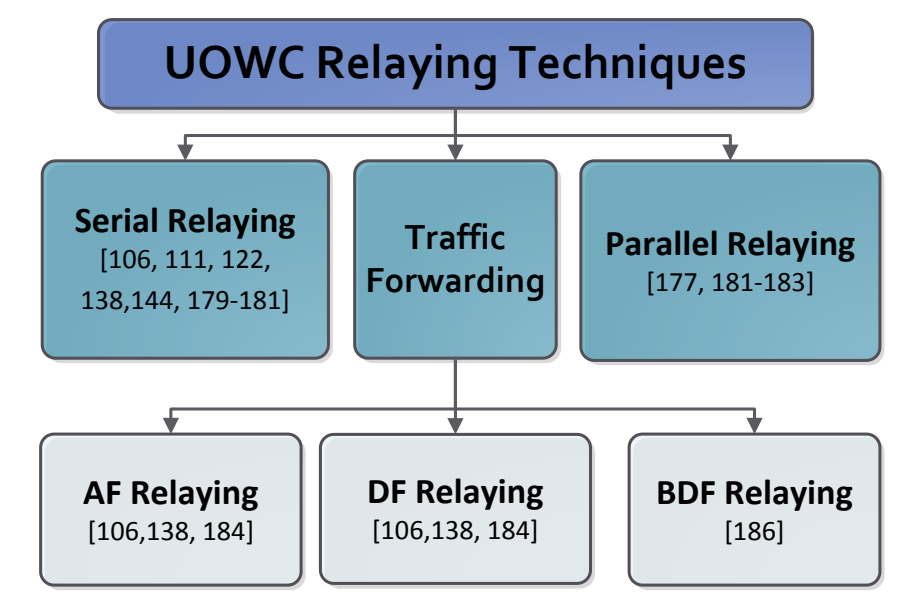

Figure 10: Classification of underwater optical wireless relaying techniques.

\subsubsection{Serial Relaying and PAT Mechanisms}

Serial transmission (a.k.a. multihop transmission) employs the relay nodes in a serial configuration along a certain routing path $[137,143,110]$, which is especially beneficial to extend the communication range and expand the cell coverage in ad hoc and cellular UOWNs, respectively. In [143], authors exploited the serial relaying to expand the coverage area of OCDMA based UOWNs. They evaluated the end-to-end performance of the proposed relay-assisted OCDMA network under absorption, scattering, and turbulence effects. In [110], endto-end BER performance of a multi-hop transmission was analytically evaluated by using single-hop BER expression as a building block. Authors in [143, 110] have applied Gauss Hermite quadrature formula and derived the closed-form BER solution under the lognormal fading channel. In [110], end-to-end BER performance is obtained by assuming that each hop experiences the same probability of error, which may not be the case in reality. Therefore, an end-to-end BER performance analysis was considered in $[137,105]$ where we have distinguished the error probability of each transmission hop.

The key point in multi-hop transmission is to employ narrowbeam light sources in order to concentrate the received signal power at the receiver aperture area. Although narrow-beam transmission significantly enhances the system performance at each hop, it requires highly directional beams and rapid PAT mechanisms which accounts for beam wander and jitters due to aquatic turbulence and random motion patterns (roll, pitch, and yaw) of the transceiver platforms [121]. Furthermore, the precision of the localization algorithms is quite decisive for positioning the complementary node in its FoV during the acquisition [179]. Lastly, a fast closed-loop tracking and wavefront control is necessary to sustain a constant link [180]. To the best of our knowledge, there is no study addressing the PAT mechanisms for UOWNs yet. When the location accuracy is low, pointing errors and misalignment could be mitigated by ensuring a certain diffusion area (proportional to the localization error) rather than directly pointing to the estimated receiver location. There- 


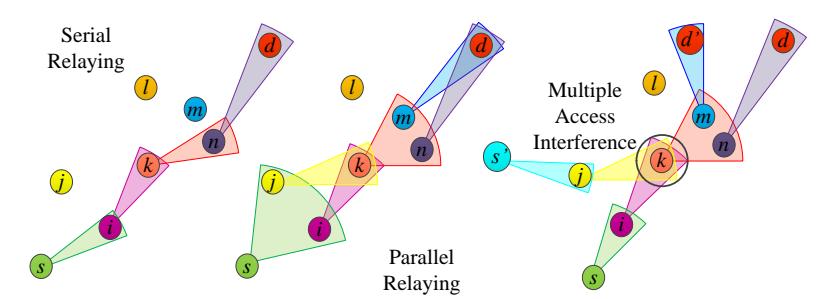

Figure 11: Illustration of serial and parallel relaying techniques along with an MAI interference scenario.

fore, it is essential to develop robust and adaptive divergence and power control schemes [181].

\subsubsection{Parallel Relaying and Relay Selection Protocols}

Parallel transmission (a.k.a. cooperative transmission) is an alternative relay-assisted transmission scheme and basically built upon the idea that the source node may be overheard by a number of neighboring nodes which can act cooperatively to relay traffic request of the source node. In other words, a set of transmitting nodes (probably each with a single optical transmitter) jointly process and transmit the traffic request by creating a virtual antenna array [182]. This cooperation naturally increases the degree of diversity and provides opportunities to mitigate multipath fading effects. Even though parallel relaying has received quite an attention in terrestrial optical wireless communications (TOWCs) (please see [177] and references therein), there is no UOWC work addressing the virtue and benefits of the cooperative relaying.

Relay selection is an interesting research topic for cooperative relaying schemes because involving all the neighbor in transmission may always not yield the desired results [183]. This is mainly because of the fundamental tradeoff between the divergence angle and received transmission power (or the communication range for a fixed power reception). In Fig. 11, for instance, relay node $\ell$ does not participate in relaying as it does not provide a better performance than involving relay nodes $m$ and $n$ only. MAI raises another issue when a relay node is incorporated with relaying to convey two different data streams as shown in Fig. 11 where node $k$ is not able to serve data streams $s \rightarrow d$ and $s^{\prime} \rightarrow d^{\prime}$ at the same time unless it employs an efficient multiple access scheme. Notice that node $k$ constitutes the bottleneck of these two data streams and such critical nodes mainly determine the overall network performance. It is important to develop adaptive divergence and power control schemes [181] for employing efficient relay selection strategies in order to sustain and improve the network performance.

\subsubsection{Traffic Forwarding Methods}

Inspired by the methods in the well-known TOWC parts, several signaling strategies can be employed for UOWCs:

Decode-and-forward Relaying. In decode-and-forward relaying, the received optical signal at each hop is converted into electrical signal, then decoded, and finally re-encoded before retransmission for the next hop. Although decode-and-forward relaying greatly improves performance as it limits background noise propagation, it may introduce significant power consumption and encoding/decoding delay to the system [105, 137, 184].

Amplify-and-forward Relaying. Amplify-and-forward relaying is conventionally realized by executing optical-electricaloptical (OEO) conversion at each node, amplifying the received signal electrically, and then retransmitting the amplified signal for the next hop. However, actual merits of amplify and forward Relaying over the decode-and-forward relaying counterpart emerges only if OEO conversion is eliminated. Alternatively, all-optical amplify-and-forward relaying process received signal in the optical domain and requires only low-speed and low-power electronic circuitry to adjust the amplifier gain [185]. The main drawback of the amplify-and-forward Relaying is the propagation of noise added at each node, which is amplified and accumulated through the path [105, 137, 184].

Bit-detect-and-forward Relaying. Different from the decodeand-forward relaying method, the relay node detects each transmitted bit of the source and forwards it to the next relay without applying any error correction [186]. Therefore, bit-detect-andforward relay can be regarded as an intermediate solution as it reduces complexity by eliminating decoding and error correction and preventing the noise propagation by detecting and forwarding the raw bits.

\subsection{Underwater Routing Techniques}

Routing holds a significant place in order to keep the UOWNs connected by discovering and maintaining the transmission routes. The physical layer issues for UOWNs are well studied in the recent past but the research on network layer issues such as routing is still in its infancy. A number of routing protocols for underwater acoustic wireless networks have been highlighted in [187, 188, 189, 190, 191, 192] some of which can be well adapted for UOWNs. The key point in adapting the existing routing protocols is that designers should take the angular sector shaped coverage region of optical nodes along with the fundamental tradeoff between the angle and radius of this sector. In what follows, we first discuss the existing routing protocols for UWONs, and then we highlight some of the potential routing algorithms proposed for underwater acoustic networks, which can also be used for UOWNs:

\subsubsection{Routing Protocols for UOWNs}

UOWNs require to design efficient and reliable routing protocols which accounts for the channel parameters of light propagation in the underwater medium. Recently, few routing protocols are developed for UOWNs which can be broadly classified into centralized and distributed schemes.

Centralized. A centralized routing scheme is proposed in [137] for UOWNs which take the underwater propagation characteristic of lightbeams into account. The proposed routing protocol in [137] assumes the availability of perfect PAT mechanisms and information of node locations. Our work is extended in [105] for location uncertainty to capture the negative impacts of pointing mismatch on the end-to-end performance multihop 


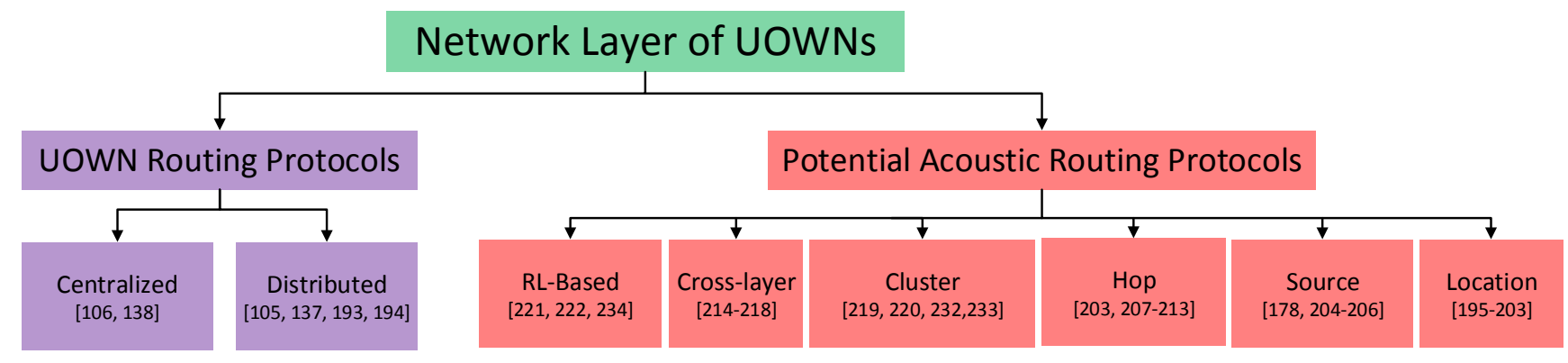

Figure 12: Existing routing protocols for UOWNs along with some potential acoustic routing schemes.

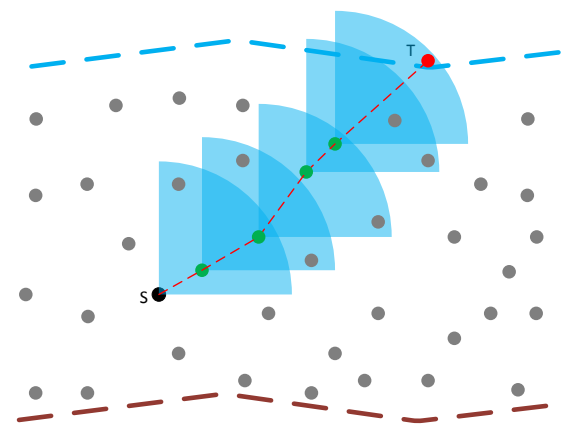

Figure 13: Illustration of distributed routing protocol for UOWNs.

UOWCs. To achieve robust and reliable links, we consider adaptive beamwidths and derive the divergence angles under the absence and presence of a PAT mechanism. We also provide end-to-end performance analysis of DF and AF relaying for performance metrics such as data rate, bit error rate, transmission power, amplifier gain, etc. Proposed shortest-path based routing protocols are tailored for optimizing a specific performance metric (e.g., data rate, BER, power consumption) while guaranteeing constraints on others.

Distributed. Although the centralized routing protocols provide better end-to-end performance, it requires the global network view that induces a high communication overhead and energy consumption across the network [193]. Accordingly, Rawan et. al. developed a distributed omnidirectional routing protocol [c.f. Fig. 13] that provides low complexity at the expense of relatively high end-to-end BER and delay in comparison with a centralized solution [193]. Similarly, we introduced a light path routing protocol (LiPaR) in [105] which takes into account the range-beamwidth trade-off and provides better endto-end performance when there is no pointing mismatch.

Alternatively, we proposed a sector-based opportunistic routing (SectOR) protocol in [194] to reap the full benefits of broadcast nature of UOWCs [c.f. Fig. 14]. Unlike routing techniques that unicast packets to a unique relay [193, 137, 105], oppor-

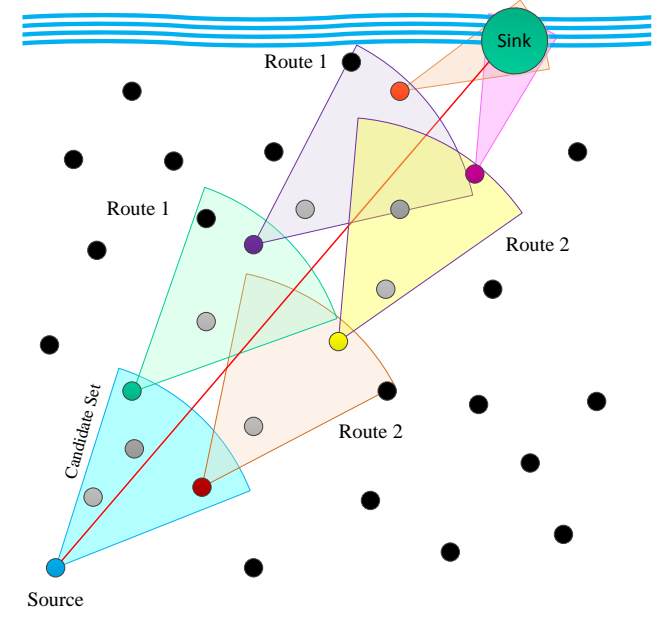

Figure 14: Illustration of SectOR protocol for UOWNs.

tunistic routing (OR) target a set of candidate relays. OR is especially suitable for UOWNs as the link connectivity can be disrupted easily due to the underwater channel impairments (e.g., pointing errors, misalignment, turbulence, etc.) and sea creatures passing through the transceivers' line-of-sight. In such cases, OR improves the packet delivery ratio as the likelihood of having at least one successful packet reception is much higher than that in conventional unicast routing. Following the performance characterization of a single-hop link, we obtain distance progress (DP) and expected (DP) metrics to evaluate the fitness of a candidate set (CS) and prioritize the members of a CS. We develop a candidate selection and prioritization (CSPA) algorithm to find the optimal sector shaped coverage region by scanning the feasible search space. Indeed, the CSPA algorithm manipulates the rate-error and range-beamwidth tradeoffs to compare different candidate sets. Simulation results show that SectOR protocol can perform even better than an optimal unicast routing protocol in well-connected UOWNs. Table 5 summarizes the features of each routing protocol for UOWNs.

\subsubsection{Potential Routing Protocols for UOWNs}

In this section, we survey some of the routing algorithms proposed for underwater acoustic networks, which can also be used 
Table 5: Comparison of existing routing protocols for UOWNs.

\begin{tabular}{|c|c|c|c|}
\hline Ref. & Location Info. & Protocol & Remarks \\
\hline $\begin{array}{l}{[137,} \\
105]\end{array}$ & All nodes & alized & $\begin{array}{l}\text { High BER performance and } \\
\text { high complexity }\end{array}$ \\
\hline [193] & $\begin{array}{l}\text { Own, neighbors, } \\
\text { destination }\end{array}$ & Dis & $\begin{array}{l}\text { Low BER performance, low } \\
\text { latency, and low complexity }\end{array}$ \\
\hline $\begin{array}{l}\mathrm{LiPaR} \\
{[105]}\end{array}$ & $\begin{array}{l}\text { Own, neighbors, } \\
\text { destination }\end{array}$ & buted & $\begin{array}{l}\text { High BER performance and } \\
\text { low complexity }\end{array}$ \\
\hline $\begin{array}{l}\text { SectOR } \\
{[194]}\end{array}$ & $\begin{array}{l}\text { Own, destina- } \\
\text { tion }\end{array}$ & Distributed & $\begin{array}{l}\text { High packet delivery ratio } \\
\text { and low complexity }\end{array}$ \\
\hline
\end{tabular}

\section{for UOWNs.}

Location based routing. The location information of underwater sensors is used in location-based routing strategy to discover the best route from the source to the destination node. In location-based routing, every node should be aware of its location, the target area, and neighbors' locations. The data is forwarded in accordance with the location information. AUV based routing protocols were proposed in [195, 196] which integrate localization and routing. An energy efficient and reliable routing protocol was introduced in [197], where the transmission from the source node starts with local flooding and then an adaptive mechanism is established to find the optimal route with minimum energy consumption. Directional flooding protocol were proposed in $[198,199]$ where the source node knows its own location, the sink location, and the location of its neighbors. The flooding region in [198, 199] was defined by the link qualities among the neighbors. The flooding phenomena can burden the network therefore, in [200] the authors have proposed a routing protocol based on focused beam. It is assumed in [200] that every node knows its location and location of the destination node, where the decision about the next hop is made at each intermediate node. Focus beam routing is a good candidate for UOWNs due to its directive nature from source to destination. However, focus beam routing should incorporate the inherent optical properties of light passing in the underwater environment. Fig. 15 shows the data forwarding scheme used in focus beam routing, where node " $A$ " is the sender node and node " $\mathrm{D}$ " is the destination node, the intermediate nodes are selected based on the cone angle $\theta$ (which can be considered as twice of the divergence angle). Nodes which lie within the cone angle $\pm \theta / 2$ of the sender node, are selected as relay nodes for forwarding the data.

A geographical reflection enabled routing protocol was introduced in [201] which tries to find the stable route between the source node and the destination node. Directional antennas were used in [201] to consider both LoS and NLoS links between the neighbor nodes. Fig. 16 shows the different scenarios for the proposed routing protocol in [201], where it can be seen from Fig. 16b and Fig. 16c that the directive and NLoS communication can help in simultaneous transmissions respectively, thus improving the throughput of the network. The proposed routing protocol in [201] was designed for underwater acoustic networks which can also be well adapted for UOWNs by incorporating the limitations of UOWC, such as absorption and scattering of light in water, turbulence, and misaligment

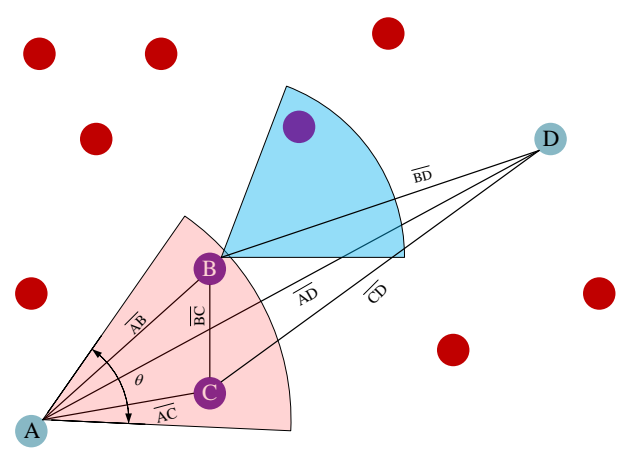

Figure 15: Illustration of focus beam routing protocol.

between the underwater transceivers. Since this routing protocol also considers the NLoS links, making it more suitable for UOWNs. Sector-based routing protocols were designed in $[202,203]$ with the location prediction of destination. The network topology in $[202,203]$ is fully mobile where each node moves along a pre-defined route. Since the sector-based protocols in [202, 203] consider mobile networks, it will be hard to implement for UOWNs due to the strict requirement of pointing and alignment between the underwater optical transceivers.

Comparative study of location-based routing protocols for underwater acoustic networks was carried out in [231]. In all of the location-based routing protocols, it is assumed that the underwater sensor nodes find its location by using GPS or by using underwater local positioning systems. However, GPS cannot work in the underwater environment, and the underwater local positioning techniques have large localization error due to the hostile underwater environment.

Source based routing. A simple and energy efficient source based routing protocol was introduced in [204]. The protocol in [204] selects the route with minimum transmission delay from source to the sink node. Once the route is defined, the nodes along the route can also transmit the data to the sink node. The average end to end delay, average energy consumption, and packet delivery ratio of the proposed protocol in [204] outperforms other traditional routing protocols. Another source based routing protocol for small size UAWC networks was proposed in [205] where each node just share information with its singlehop neighbor nodes and find a minimum cost path from source to the destination. Source based energy efficient routing protocols have also been developed in [206, 178] which consider a layered architecture from the source node to the sink node. In layer based routing protocols, the surface sink first assigns layers to each underwater sensor node where the layers are defined based on the number of power ranges the surface sink can use as shown in Fig. 17. Once the layers are defined for each node, the routing path between the source node and the surface sink is established by forwarding nodes $\left(F_{1}\right.$ and $\left.F_{2}\right)$ as shown in Fig. 17. The forwarding nodes are defined based on the communication range of the source $\left(r_{1}\right)$, the communication range of the next forwarding node $\left(r_{2}\right)$, and the distance to the surface sink. The layer based routing protocols work well in case of UANs as the communication range is omnidirectional. How- 
Table 6: Comparison of potential routing protocols for UOWNs.

\begin{tabular}{|c|c|c|c|c|}
\hline Literature & Location information & Protocol type & Knowledge required & Remarks \\
\hline$[195,196]$ & Own and destination & Location based & Self and sink node location & Integrates routing and localization \\
\hline [197] & Own and destination & Location based & Self and sink node location & Reliable and energy efficient \\
\hline$[198,199]$ & $\begin{array}{l}\text { Own, neighbors, and } \\
\text { destination }\end{array}$ & Location based & $\begin{array}{l}\text { Self, neighbors, and sink node } \\
\text { location }\end{array}$ & $\begin{array}{l}\text { Reliable, reduce end to end delay, and } \\
\text { avoid packet collisions }\end{array}$ \\
\hline [200] & Own and destination & Location based & Self and sink node location & $\begin{array}{l}\text { Cross layer design which integrate } \\
\text { routing, MAC, and physical layers }\end{array}$ \\
\hline$[201]$ & Own and destination & Location based & Self and sink node location & $\begin{array}{l}\text { Use of NLoS communication for } \\
\text { routing }\end{array}$ \\
\hline$[202,203]$ & Own only & Location based & Self location only & $\begin{array}{l}\text { Provide dynamic routing for a mobile } \\
\text { network }\end{array}$ \\
\hline$[204,205]$ & Not required & Source based & Transmission delay & $\begin{array}{l}\text { Minimize end to end delay, reliable, } \\
\text { and energy efficient }\end{array}$ \\
\hline$[206,178]$ & Not required & Source based & $\begin{array}{l}\text { Number of power ranges and } \\
\text { communication range of for- } \\
\text { warding nodes }\end{array}$ & $\begin{array}{l}\text { Minimize end to end delay, reliable, } \\
\text { and energy efficient }\end{array}$ \\
\hline$[207,208]$ & Not required & Hop based & Channel awareness & $\begin{array}{l}\text { Increase lifetime and minimize end to } \\
\text { end delay }\end{array}$ \\
\hline$[209,210]$ & Not required & Hop based & Channel and depth awareness & $\begin{array}{l}\text { Increase lifetime and minimize end to } \\
\text { end delay }\end{array}$ \\
\hline [211] & Not required & Hop based & Multiple antennas & Multiplexing and diversity gain \\
\hline$[212,213]$ & Not required & Hop based & Weighting strategy & Energy efficient and topology aware \\
\hline$[214,215]$ & Not required & $\begin{array}{l}\text { Cross layer hop } \\
\text { based }\end{array}$ & $\begin{array}{l}\text { Transmission delay, distance to } \\
\text { sink, channel conditions, and } \\
\text { buffer size of the forwarding } \\
\text { node }\end{array}$ & Utilization of the channel efficiently \\
\hline $\begin{array}{l}{[216, \quad 217,} \\
218]\end{array}$ & Not required & $\begin{array}{l}\text { Cross layer hop } \\
\text { based }\end{array}$ & $\begin{array}{l}\text { Link quality and channel con- } \\
\text { ditions }\end{array}$ & Multipath routing and power control \\
\hline$[219,220]$ & Own and destination & Cluster based & $\begin{array}{l}\text { Number of clusters and loca- } \\
\text { tion of self and sink node }\end{array}$ & $\begin{array}{l}\text { Energy efficient and low end to end } \\
\text { delay }\end{array}$ \\
\hline$[221,222]$ & Not required & Hop based & $\begin{array}{l}\text { Residual energy information of } \\
\text { self and neighbor nodes }\end{array}$ & $\begin{array}{l}\text { Energy efficient and life time en- } \\
\text { hancement }\end{array}$ \\
\hline $\begin{array}{l}{[223, \quad 224,} \\
225, \quad 226, \\
227, \quad 228, \\
229,230]\end{array}$ & Own and sink & Hop based & $\begin{array}{l}\text { Number of power ranges and } \\
\text { communication range of for- } \\
\text { warding nodes }\end{array}$ & $\begin{array}{l}\text { Reduce power consumption and end } \\
\text { to end delay }\end{array}$ \\
\hline
\end{tabular}

ever, it can be modified to suit UOWNs such that the forwarding nodes are defined based on the directive links from the source node to the surface sink. Moreover, light passing through different layers of the seawater suffers from different absorption and scattering, which needs to be taken into account. Also, PAT mechanisms are required to align the underwater optical transceivers for layer-based routing protocols.

Hop-by-hop routing. In hop-by-hop routing, the intermediate nodes (or relay nodes) selects the next hop by itself. Hopby-hop routing provides flexibility and scalability to the network but the route selection may always not be optimal. Channel aware hop-by-hop routing protocols were introduced in [207, 208] where the speed of acoustic waves in different depth were taken into consideration for the relay nodes to reduce the end to end transmission delay. In [203], the authors have proposed a hop-by-hop routing protocol based on beam-width and direction of the intermediate nodes. Adaptive depth based routing protocols were introduced in [209, 210] which takes into account the speed of acoustic waves at different depth levels, depth of sink node, and distance to sink node. Although the adaptive depth based routing protocols work well for underwater acoustic communication, it may fail for optical communication since light is sensitive to the distinct turbidity level at different depths [46]. A MIMO-OFDM based routing protocol was introduced in [211] to take the advantage of multiplexing and diversity gain adaptively. The proposed cross-layer design in [211] adapts itself to the noise and interference for underwater acoustic channels and selects a suitable transmission mode for the subcarriers. An energy efficient and network topology aware greedy routing protocol was proposed in [212] which assigns adaptive weights to the highly connected nodes. For underwater delay tolerant networks a redundancy-based routing protocol was designed in [213] which adopts a tree-based forwarding method to replicate packets. Hop-by-hop routing protocols are good candidates for UOWNs due to their distributed nature for candidate selection. They can improve the end to end reliability at the expense of high time delay [193].

Cross Layer routing. The cross-layer routing protocols take the information available from different layers into account and provide a solution to several networking issues such as scheduling, defining routing policy, and power control. Cross-layer routing protocols can also select the next hop for transmission by considering the transmission delay, distance to sink, channel conditions, and buffer size of the candidate node. Crosslayer strategy increases the overall network performance and minimizes the energy consumption of the network. Cross-layer 


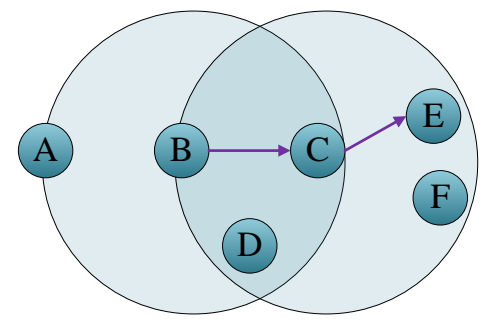

(a) Multihop omnidirectional optical links.

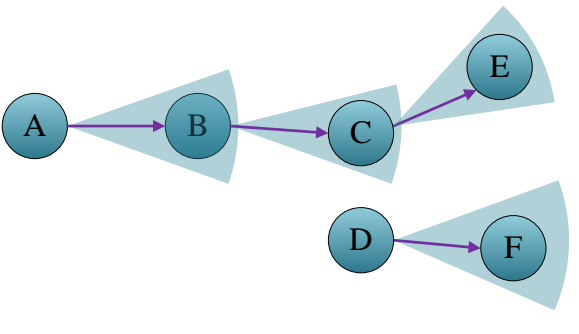

(b) Multihop directional optical links.

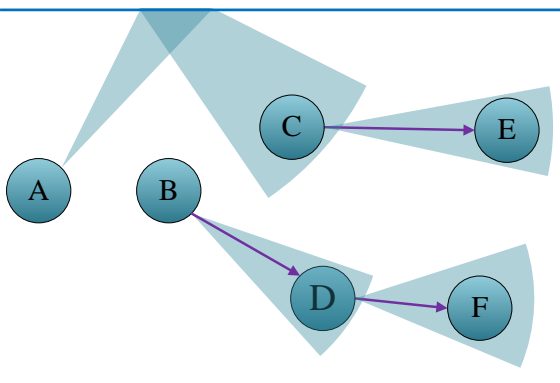

(c) Multihop directional optical links with reflection.

Figure 16: Multihop underwater routing protocols: a) Omnidirectional, b) LoS directive, and c) NLoS directive (Reflective).

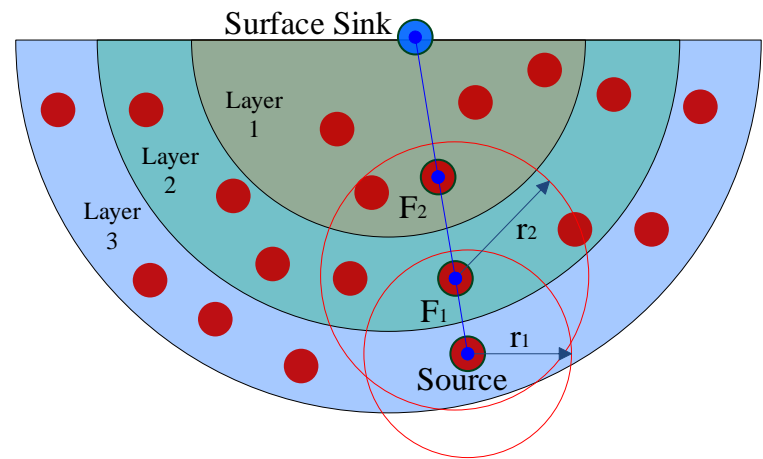

Figure 17: Illustration of layer-based routing.

protocols for the 3D underwater environment were investigated in $[214,215]$ which utilizes the channel efficiently and sets the optimal packet size for transmission. Multipath power control routing protocols were proposed in $[216,217]$ which combine multipath routing and power control at the sink node. Channel aware cross-layer routing protocols are also investigated in [218] which exploits the link quality for the relay selection. All of these cross-layer protocols consider the interaction between the underwater acoustic channel and routing functions. However, the UOWC channel is different than the acoustic channel, and therefore, its impediments need to be taken into account, such as absorption and scattering of light, turbulence, and water depth.

Clustered routing. Cluster based routing is especially suited for infrastructure based UOWNs as shown in Fig. 3. In cluster based routing, the network is divided into a number of clusters/cells based on the geographical location of the nodes. Once the network is divided into clusters, the cluster head (i.e., $\mathrm{OAP} / \mathrm{OBS}$ ) is selected for each cluster by using any cluster head selection strategy. The cluster head is used as a gateway to communicate between the clusters and to the sink node. A locationbased routing protocol was introduced in [219] which divides the network into clusters and the data from the nodes are gathered by the cluster heads. A distributed clustering based protocol was proposed in [232] where the communication between the cluster head and the sensor node was single hop. Location unaware cluster based multihop routing protocol was proposed in [220] where the sensor nodes do not know their location and location of the cluster head. The interested readers are referred to [233] where a number of cluster based routing protocols are highlighted for underwater wireless sensor networks.

Reinforcement learning based routing. The routing protocols based on reinforcement learning uses Q-learning method for the network states and adapts itself to the topology change. The node analyzes its remaining energy and energy of its neighbor nodes, applies a reinforcement function, and then selects the optimal node to forward the data [221]. The routing problem in [221] is fully distributed and formulated as a Markov decision process where the state space consists of all the nodes. A machine learning based routing protocol was proposed in [222] which is energy efficient and improves the lifetime of the network. A layer structured based routing protocol was introduced in [234] for hybrid acoustic and optical architecture where the upper layer cluster heads supervise the routing in lower layer by using the Q-learning function. These routing protocols try to improve the lifetime, energy efficiency, latency, and reliability for UWSNs. Therefore these protocols are well-suited for UOWNs by taking into account the UOWC channel parameters, such as misalignment between the transceivers, absorption and scattering, turbulence, and field of view.

Energy efficient routing. Designing energy-efficient UWSN is of utmost importance for real-life underwater applications, which is especially a challenging task if the engineering hardship and monetary cost of battery replacement in the harsh underwater environment are taken into account. Therefore, network lifetime is a critical performance metric for routing protocols which can be improved by reducing the energy consumption of the network. Adjustment of the sleep/awake mode of sensors is a proven technique to improve the lifetime of the network. Hence, the authors in [235] modeled the sleep interval for underwater sensors in opportunistic routing to improve the network lifetime. The interested readers are referred to $[236,237,238]$ for duty cycle optimization to increase the network lifetime. An ultimate solution for prolonging the network lifetime can be done via provisioning energy self-sufficient nodes by harvesting ambient renewable energy from natural sources in the aquatic environment and storing it in an energy 
buffer. As surveyed in [239], ongoing research efforts on terrestrial communications have shown that energy harvesting plays a significant role in enhancing the network lifetime. In the realm of UOWNs, we proposed a muti-source energy harvesting system in $[45,184]$ where energy is harvested from multiple underwater sources such as acoustic resonators and microbial fuel cells (MFCs) [240]. It is shown that network connectivity is mainly determined by the active nodes with sufficient energy while localization accuracy improves with the increasing degree of connectivity. Reminding that location accuracy is critical information for better end-to-end performance via precise pointing and alignment [105], energy efficiency and availability is one of the most crucial design consideration to be taken into account.

\subsection{Summary and Insights}

The limited communication range and directivity of UOWCs require novel network layer protocols for relaying and routing tasks. Therefore, in the first part of the section, we have briefly discussed the possible relaying mechanisms in order to extend and expand the range and coverage of ad-hoc and infrastructure based UOWNs, respectively. Two different relaying mechanisms are presented; serial relaying and parallel relaying. In the serial relaying, the path between the source and destination nodes is established by considering a single forwarding node at each hop. On the other hand, the parallel relaying admits multiple relay nodes at each hop, which cooperates with each other in selecting and transmitting to the next hop towards the destination. Thanks to narrow and focused light beams at each hop, serial relaying can significantly enhance the overall endto-end system performance. However, serial relaying requires to develop reliable PAT mechanisms in order to avoid performance degradation because of the pointing errors and misalignment. Unlike the serial relaying, parallel relaying operates on wider beam angels to cover multiple nodes, hence, provide a more reliable connectivity as it does not necessitate precise PAT mechanisms. Furthermore, parallel relaying increases the diversity of the system and mitigate the multipath effect at the expense of MAI avoidance or mitigation complexity. Therefore, serial relaying can be employed for high-speed applications whereas parallel relaying can be adopted for low-speed applications seeking for high reliability.

Three different types of traffic forwarding methods including DF, AF, and BDF have also been discussed. While the DF method improves the system performance as it reduces the noise propagation in return for higher power consumption, the AF method is less complex and more power efficient in return for noise propagation along the routing path. BDF is an alternative solution with a preferable cost performance index since BDF relays detect transmitted bits and forward to the next hop without decoding.

The limited communication range and directivity of UOWCs set network connectivity as one of the main performance delimiters of UOWNs. Even though the literature on routing protocols for underwater acoustic communications is quite rich, very few works faced the challenges posed by light propagation in the aquatic medium. Therefore, the second part of this section covers the existing routing protocols for UOWNs and discusses the potential underwater acoustic routing protocols that can be used for UWONs. All of these protocols can be modified to be exploited in UOWNs by taking into account the limitations of UOWNs such as directivity and limited communication range. Noting that there is no routing protocol to the best option for all circumstances, Table 6 compares the different possible routing protocols.

\section{Transport Layer: Connectivity, Reliability, and Flow/Congestion Control}

Unlike the first two lower layers, transport layer of UOWNs is still in a primitive stage and remains totally unexplored. Therefore, this section discusses the fundamental challenges for developing an efficient transport layer including connectivity, reliability, flow control, and congestion control aspects of UOWNs. Classification of transport layer challenges along with the references is presented in Fig. 19.

\subsection{Requirements and Design Challenges}

The research on transport layer problems for UOWNs is not active. However, the transport layer protocols for UOWNs should consider the following requirements and design challenges.

Connectivity. In addition to its critical importance for lower layers and localization, connectivity poses unique challenges in designing suitable transport layer protocols for UOWNs. The degree of connectivity in UOWNs is quite low because of the absence of bidirectional links which hinders the implementation of the essential transport layer functions. One can mitigate such problems by employing hybrid acoustic-optic systems which exploit the acoustic signals merely for control signaling. Alternatively, creating an omnidirectional control coverage can help the coordination among the neighboring nodes [c.f. Fig. 8] that can be realized by equipping the node with multiple lowcost low-power transceivers to operate at short-distances and wide beamwidths.

Reliability. Reliability is especially essential for missioncritical applications which require flows to be forwarded with a specific rate and limited packet loss. Therefore, sustaining these requirements requires the integration of transport and routing protocols. In this sense, opportunistic routing is a potential candidate since it allows multiple nodes to listen to the transmit data and forward it to the next hop in a prioritized way. In this manner, the packet delivery ratio is substantially enhanced since lower priority nodes start transmission if the higher priority nodes fail. Moreover, the unique characteristics of UOWNs make it not possible to use existing TCP and UDP protocols in a plug-and-play fashion. Therefore, there is a gap in the literature to develop transport layer protocols that tackle such kind of limitations of UOWCs by leveraging the advantages of opportunistic routing. 


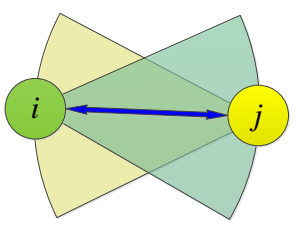

Bidirectional Link

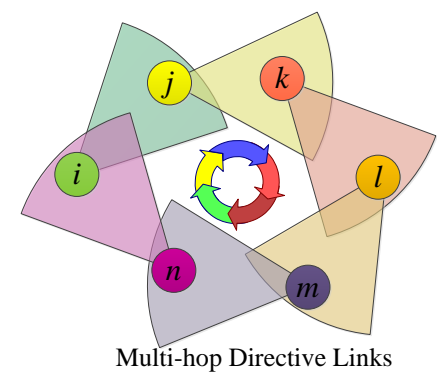

Multi-hop Directive Links

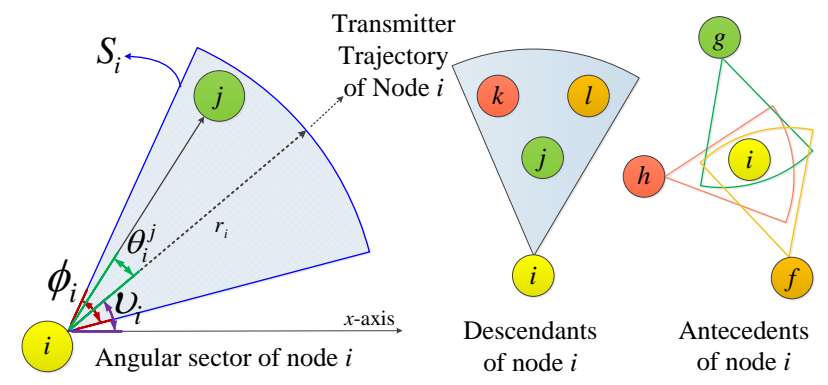

(a) Connection types in random sector directed graphs.

(b) Connectivity parameters, descendants, and antecedents of $n_{i}$.

Figure 18: Depiction of connection types, connection parameters, descendants, and antecedents for random sector directed graphs.

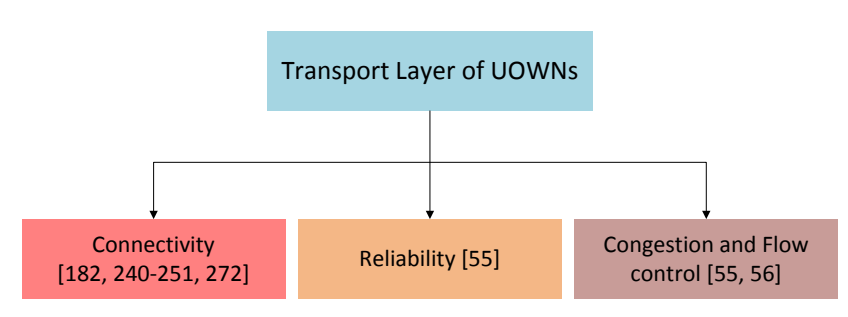

Figure 19: Classification of transport layer challenges.

Congestion Control. In order to maintain the traffic flow and avoid congestion in the network, congestion and flow control mechanisms should be investigated. The distinct features of UOWNs such as link failures and dynamically changing connectivity patterns are the main challenges to design effective control mechanisms, which are not explored until yet.

\subsection{Connectivity analysis of UOWNs}

Connectivity of UOWNs is the most critical component of transport layer as other network functions heavily depend on a connected network assumption. It is also used as a metric for different performance parameters such as survivability, robustness, and fault tolerance [241]. Connectivity is measured by the number of links in the network and a network is referred to be as connected if there exists at least one connecting path between any two nodes in the network. In strongly connected networks bidirectional links exist between any pair of nodes while in a directed network the links are usually unidirectional until and unless both nodes are in the beam scanning angle of each other [242]. Moreover, bidirectional links can also be achieved by using different frequencies [243] and polarization [244] of optical light. The problem of network connectivity for omnidirectional networks is addressed in [245]. The exact closed-form analytical expression of connectivity in multihop wireless networks for physical layer parameters still remains as an open research problem. The connectivity parameters of UOWNs depend on the transmission range of optical sensor nodes, number of optical sensor nodes, number of descendants and antecedents, node orientation, and the beam width [c.f. Fig. 18b].

Range limitation of UOWC can be augmented with multihop UOWNs where nodes can share information for long dis- tances through intermediate nodes. Indeed, multi-hop cooperative communications have been extensively studied for RF networks [182], underwater acoustic networks [246], and TOWNs [247]. Due to the omnidirectional communication capability of RF and acoustic signals, wireless sensor networks are traditionally modeled as geometric random graphs [248] where two sensor nodes $n_{i}$ and $n_{j}$ are generally assumed to establish a bidirectional communication link (i.e., $n_{i} \leftrightarrows n_{j}$ ). On the contrary, such a model is not suitable for UOWNs because a node can only reach to the nodes within a certain beam scanning angle around their transmission trajectory, that is, optical wireless nodes are connected via unidirectional links. Directed communication networks are generally modeled by random scaled sector graphs [249] where a unidirectional communication link from node $n_{i}$ to $n_{j}$ (i.e., $n_{i} \rightarrow n_{j}$ ) is established if and only if $n_{j}$ is positioned within the beam scanning angle of $n_{i}$. Notice that a directed reverse path is possible (i.e., $n_{j} \rightarrow n_{i}$ ) if $n_{i}$ is in the beam-width of $n_{j}$ or through other multi-hop path as illustrated in Fig. 18a. A connectivity framework for multihop UOWNs was discussed in [250] where the authors have assumed bidirectional links between every pair of optical sensor nodes. In $[251,252]$, we have analyzed the connectivity of UOWNs by using random sector graphs where we have considered unidirectional links between underwater optical sensor nodes.

In order to define a random sector graph, we consider the total number of optical nodes are $m$ and the scanning sector (coverage area) of $n_{i}, 1 \leq i \leq m$, which is defined as a tuple of random orientation $\zeta_{i}$, scanning angle $\phi_{i}$, communication range $R_{i}$, and sensor node coordinates $\mathbf{c}_{i}$, i.e., $\mathbf{S}_{i}=\left(\zeta_{i}, \phi_{i}, R_{i}, \mathbf{c}_{i}\right)$ which is illustrated in Fig. 18b. Accordingly, UOWNs can be defined as a random sector directed graph $\mathcal{G}(\mathcal{V}, \mathcal{E})$ where $\mathcal{V}=\left\{\mathbf{c}_{1}, \ldots, \mathbf{c}_{i}, \ldots, \mathbf{c}_{M}\right\}$ represents the set of vertices and $\mathcal{E} \in\{0,1\}^{M}$ is the set of links which is primarily characterized by $\mathbf{S}=\mathbf{S}_{1}, \ldots, \mathbf{S}_{i}, \ldots, \mathbf{S}_{M}$. Notice that $\mathcal{E}_{i, j}=1$ only if $n_{i} \rightarrow n_{j}$ holds. Random sector directed graphs and random geometric graphs are identical in case of $\phi=2 \pi[242,273,241,249]$. Notice that two nodes $i$ and $j$ are connected when the distance between them is less than $R$ in random geometric graphs, however, the connectivity of random directed sector graphs also depends on the beam scanning angle and its orientation. Fig. 20a and Fig. 20b shows two different random directed sector graphs with scanning angles of $\phi=\frac{\pi}{3}$ and $\phi=2 \pi$, respectively. It is obvious that increasing the scanning angle for each node from 


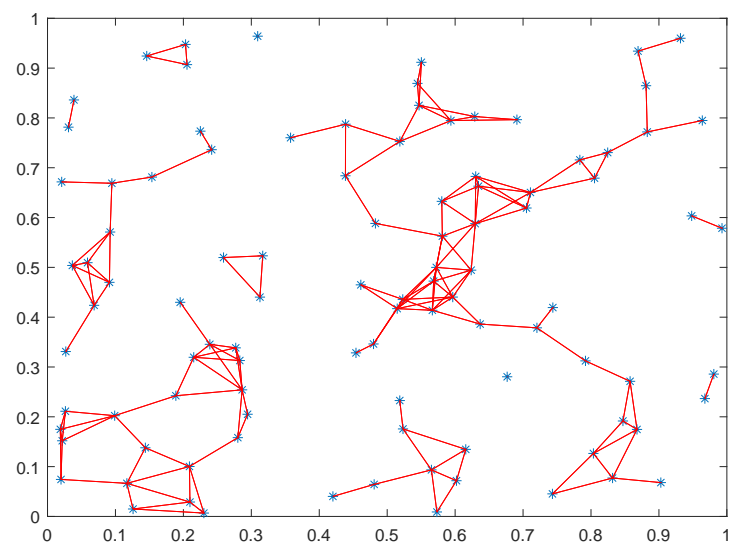

(a) $m=100, \phi=\frac{\pi}{3}$, and $R=0.2 m$.

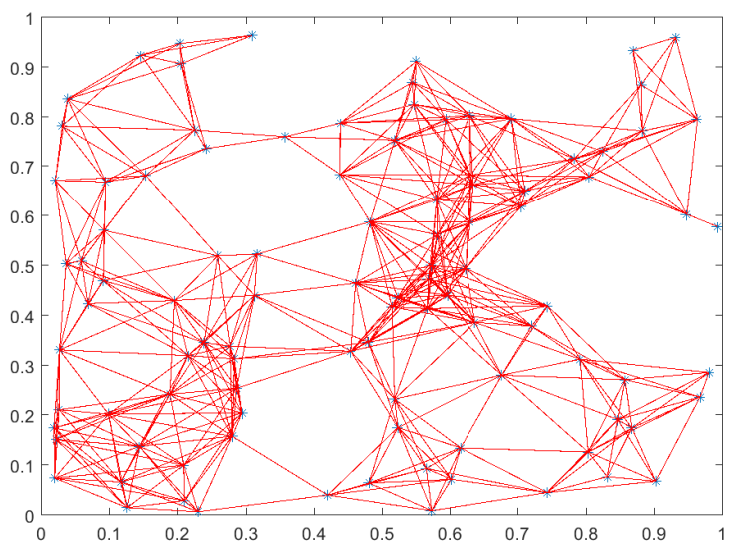

(b) $m=100, \phi=2 \pi$, and $R=0.2 m$.

Figure 20: Illustration of random directed graph scenarios for different parameters.

Table 7: Comparison of connectivity analysis of wireless communication systems

\begin{tabular}{|l|l|l|l|}
\hline \hline Literature & Channel model & Link type & Graph model \\
\hline$[253,254,255,256,257,258,259,260]$ & Terrestrial RF & Bidirectional & Random graphs \\
{$[261,262,263,264,265]$} & Underwater acoustic & Bidirectional & Random graphs \\
{$[266,267,268,269,270,271]$} & Terrestrial optical & Bidirectional/Unidirectional & Random graphs/Random sector graphs \\
{$[250,48,272,251,252]$} & Underwater optical & Bidirectional/Unidirectional & Random sector graphs \\
\hline \hline
\end{tabular}

$\phi=\frac{\pi}{3}$ to $\phi=2 \pi$, increases the number of links in the graph. These asymmetric and directional characteristics of the random directed sector graphs require us to define descendant and antecedent neighbors for every node. The descendants of node $n_{i}$ are defined as $\mathcal{D}_{i} \triangleq\left\{n_{j} \mid \forall j: \mathcal{E}_{i, j}=1\right\}$, i.e., the set of nodes who lies within the coverage region of $n_{i}$, antecedents of $n_{i}$ are defined as $\mathcal{A}_{i} \triangleq\left\{n_{j} \mid \forall j: \mathcal{E}_{j, i}=1\right\}$ the set of nodes who can reach to $n_{i}$. In Fig. 18b, the set of descendants and antecedents of $n_{i}$ are shown as $\left\{n_{j}, n_{k}, n_{l}\right\}$ and $\left\{n_{g}, n_{h}, n_{f}\right\}$, respectively.

The closed form expression for the connectivity of UOWNs was derived in $[251,252]$ which takes into account various parameters of the UOWC channel such as transmission range, beam scanning angle, and network density. To show the impact of these parameters numerically, geometric/photon limited path loss model with LoS links were considered. We considered networks of $m=100$ and $m=500$ optical sensor nodes randomly deployed in underwater $100 m \times 100 m$ square area respectively. The probability of a connected network is evaluated when each node is connected to at least one node $(k=1)$ and when each node is connected to at least two other nodes $(k=2)$. The transmission range $R$ varies from 1 to 20 meters and we set the beam scanning angles of the nodes with different widths of $\phi=\frac{2 \pi}{9}, \frac{\pi}{2}, \frac{3 \pi}{4}$, and $2 \pi$ to see the impact of scanning angles on the probability of a connected network. Fig. 21a Fig. 21d shows that increase in the beam scanning angles, number of nodes, and transmission range results in high probability of a connected network. Table 7 summarizes the literature on connectivity analysis of different wireless networks.

\subsection{Reliability}

Packet losses may occur during the transport as a result of the hostile underwater channel impairments and network conges- tion. Hence, transport protocol can check the data corruptions by means of error correction codes and verify the correct receipt by the ACK/NACK messages to the source node. Considering the relation between a node and its antecedents and descendants as described above, optical sensor nodes may always not be able to convey ACK/NACK messages to its antecedents. That is, operation of such a mechanism requires a fully connected network such that there is always another communication path to deliver ACK/NACK messages to the source node. Hence, it is essential to handle shadow zones where temporary connectivity loss and high bit error rates occur [55]. Transmission control protocol (TCP) is the best-known connection-oriented transport layer protocol which assumes congestion as the only cause of packet loss and reduces the rate if packet losses occur. However, obstruction, pointing and misalignment events are quite common in UOWCs and an efficient UOWN transport layer protocol must distinguish between packet losses due to the congestion and channel impairments. Alternatively, user datagram protocol (UDP) is a connection-less transport layer protocol which may suite the UOWN better for very simple transmission applications. Rather than traditional end-to-end approaches, reliability can also be characterized in a hop-byhop fashion. However, a hop-by-hop based reliability may not guarantee an end-to-end reliable network. Therefore, UOWNs paradigm necessitates novel transmission protocols which ensures the reliability by accounting for the underwater channel impairments and limited connectivity of UOWNs.

\subsection{Congestion and Flow Control}

Congestion control is needed in order to avoid from being congested due to oversubscription of many traffic flows which may not be affordable by available network capacity whereas 

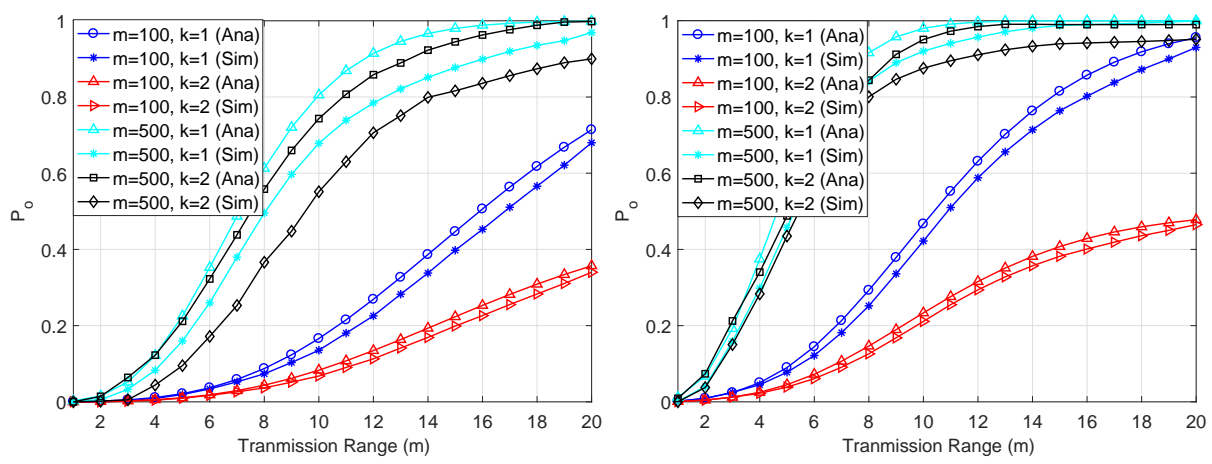

(a) Probability of connectivity vs. transmission (b) Probability of connectivity vs. transmission range for $\phi=\frac{2 \pi}{9}$.

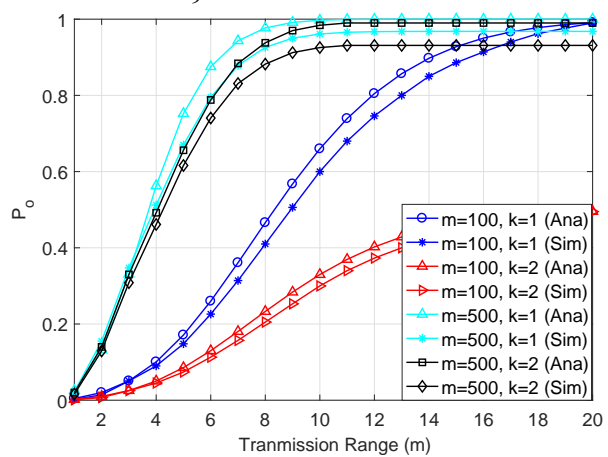
range for $\phi=\frac{\pi}{2}$.

(c) Probability of connectivity vs. transmission (d) Probability of connectivity vs. transmission range for $\phi=\frac{3 \pi}{4}$.

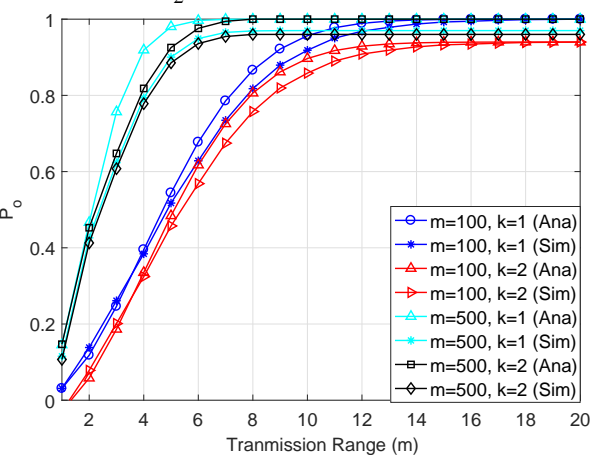
range for $\phi=2 \pi$.

Figure 21: Probability of connectivity vs. different transmission angles and ranges.

flow control is required to manage the sender's transmission rate in order to prevent buffer overrun at the receiver. Due to the window-based mechanism which relies upon the accurate round trip time (RTT), most of the TCP implementations are unsuited for underwater acoustic networks as they incur end-to-end delay with high mean and variance [55]. Even if UOWCs provide very high propagation speeds, a potential transport protocol still needs to take the link failures because of the dynamic topology changes of UOWNs into account. Alternatively, rate-based transport protocols do not depend on windows-based mechanism and can provide a flexible rate control, however, it requires feedback messages to dynamically adapt the transmission rate according to the packet losses. The rate-based scheme is not appropriate for UOWNs due to high mean and variance of feedback delay [56] where some of the UOWN nodes may not receive any feedback messages if there is not a connecting path from the receiver to the transmitter in case of limited connectivity. Accordingly, proposed congestion and flow control mechanisms should account for such kind of specific challenges related to UOWNs. It is especially important to leverage critical information from lower layers to predict and handle shadow zones as connectivity can be regarded as the main delimiter of any potential transport layer protocol.

\subsection{Summary and Insights}

In this section, we have first discussed the connectivity issue of UOWNs which is an essential parameter to deploy a fully operational network. Connectivity can be regarded as one of the major problems of optical wireless networks as it requires not only the LoS link between two nodes for communication but also require the directionality information. Connectivity of the directional networks can be evaluated by modeling the network as random sector graphs where the links are unidirectional. The probability of having a connected UOWN has been evaluated as a function of important hardware parameters such as the beam scanning angle, transmission range, and the node density. Therefore, fine tuning of all these three parameters is required to achieve a high probability of a connected network. In Table 7, we have compared the connectivity analysis of different wireless networks based on the channel model, link type, and graph class.

The second part of this section focuses on the issue of reliability in UOWNs where there is a high chance of packet loss due to different obstacles such as hostile underwater optical channel, unidirectional communication, and network congestion. In transport layer protocols, it is important to check the quality of the data and inform the sender node about the quality of the data by sending ACK/NACK messages. However, conveying ACK/NACK messages in unidirectional networks since the sender node and the receiver node may not be fully connected, i.e., a unidirectional link from the sender to receiver. The possible choices of transport layer protocol for UOWNs are TCP and UDP. TCP may not be the best-suited option for UOWNs because of two main limitations: 1) It is a 
connection-oriented protocol and connectivity of UOWNs may not be guaranteed all the time and 2) TCP merely considers congestion as a cause of packet loss, however, underwater optical wireless channel impairments have a significant impact on data losses in UOWNs. We pointed out the hybrid optical-acoustic underwater networks as a potential remedy to overcome above limitations of connection-oriented transport protocols. On the other hand, UDP is rather more suited to UOWNs thanks to its simplicity and hop by hop reliability. However, it cannot be used for applications which require an end-to-end reliability. Therefore, it is necessary to develop transport layer protocols for UOWNs which account for the underwater channel impairments, provide end-to-end reliability, and can take advantage of the information from the lower layers.

Lastly, we have discussed the possible congestion and flow control mechanisms for UOWNs. The possible protocols for congestion and flow control in UOWNs are based on time and rate. The time-based protocols (e.g., RTT) are not suited for UOWNs due to the dynamic nature of UOWC link while the rate based protocols can provide flexible rate control but it requires the feedback messages to adapt the transmission rate according to the packet losses. Additionally, because of the limited connectivity issue, it may not be feasible to get feedback messages from the sender and thus adopting the transmission rate may not be possible. Therefore, the existing congestion and flow control mechanisms used for underwater networks cannot be directly applied to the UOWNs and needs to be modified.

\section{Application Layer}

Even though one can count numerous applications for UOWNs, application layer protocol is a completely unexplored area of research. As being the top layer, lower layers serve according to the needs of application layer to complete certain tasks. Therefore, it can be regarded as an abstraction layer that masks the rest of the application from the processes in the lower layers such as transmission, routing, congestion control, etc. Next, we present a guideline on requirements and challenges of UOWN application layer:

\subsection{Requirements and Design Challenges}

The main purpose of a potential application layer protocol is multifold and should have following functionalities:

- To provide a mediating language to query the entire UOWNs and advertise events and assign the tasks.

- To provide efficient network management tools which can see and manipulate the hardware and software features of the lower layers.

- To recognize communication partners, provision the demanded QoS as per the resource availability, and realize synchronization if needed.

- To ensure source and destination nodes are identified, reachable, and ready to send and receive data, respectively.
- To authenticate both ends for security purposes and make sure the existence of an agreement about error recovery mechanisms, data integrity, and privacy.

Having these functionalities in the hand, application layer protocols are needed to be customized according to the QoS requirements of target applications. We refer interested readers to [274] for detailed information regarding the potential UOWSN applications.

\section{Localization in Underwater Optical Wireless Networks}

Numerous acoustic based localization techniques are well investigated in the past since localization is important for tagging the data, detection of an underwater object, tracking of underwater nodes, underwater environment monitoring, and surveillance. Nevertheless, due to the challenges discussed in previous sections for each layer of UOWNs, there is a dire need to develop novel localization techniques for UOWNs. Therefore, this section provides the fundamental concepts of underwater localization, state of the art underwater localization systems, and development of localization techniques for UOWNs.

\subsection{Challenges for Underwater Localization}

Localization of underwater sensors is an important part of UOWNs for many applications such as resource exploration, surveillance, underwater environment monitoring, and disaster prevention. The large propagation delay of acoustic channels and high attenuation of RF/optical channels pose significant challenges for underwater localization. The major challenges for underwater localization are

- Deployment of nodes: Most of the localization algorithms depend on the distribution of sensor nodes and the anchor nodes to form a network [275, 276]. Deployment of sensor nodes in the harsh underwater environment is a challenging task.

- Mobility of the nodes: Due to the uncontrollable phenomena such as winds, turbulence, and current, the underwater sensor nodes inevitably drifts from their actual position. The location of anchor nodes on the surface buoys can be accurately measured by using GPS but the location of the underwater nodes cannot be precisely measured [277, 276].

- Harsh underwater channel: Variations in the underwater wireless communication channel is very severe for all type of carrier waves. The effects of attenuation, absorption, reflection, scattering, and noise do not allow for accurate range measurements, thus reflecting large localization estimation error [278]. For intensity-based localization systems, however, if one is able to analyze the ballistic photons of a reference signal, it can lead to accurate range measurements [279].

- Synchronization: As the GPS signals are not available in the underwater environment, it is hard to achieve the 
time synchronization between the sensor nodes. Thus, if the time of arrival based ranging is used, this misssynchronization will lead to large localization error [280, 281, 282].

\subsection{Localization for UOWNS}

UOWNs localization is one of the major research areas nowadays because of the development of high-speed UOWC systems. Localization in terrestrial wireless networks has been studied widely and detailed surveys are presented on this topic [298, 299, 300, 301, 302, 303]. Nevertheless, GPS and all of these RF-based localization schemes cannot work in the underwater environment. Thus, many researchers developed localization schemes for the underwater environment based on acoustic waves. Localization of underwater acoustic networks have also been studied widely in the past and number of surveys are presented on this subject [304, 277, 276, 278, 305]. Since the localization techniques used for terrestrial wireless networks and underwater acoustic networks cannot be directly applied to UOWNs, novel localization schemes have recently been developed for UOWNs. In this section, we cover the the literature on optical ranging techniques and localization algorithms for UOWNs.

\subsubsection{Optical Ranging}

Every underwater localization algorithm requires distance estimation between the nodes or between the node and anchors. The distance is estimated by using optical ranging for UOWC systems. Optical light passing through the aquatic medium suffers from widening and attenuation in angular, temporal, and spatial domains [297]. In the literature, only time of arrival (ToA) and received signal strength (RSS) based localization techniques exist for UOWNs. In [297] the authors have proposed an underwater optical positioning system, where an OBS was considered as an anchor node which transmits optical signals. The sensor nodes receive the optical signals from multiple anchors and locate itself using simple linear least square solution. In [120], an RSS based distance estimation technique is developed for UOWNs for a given data rate. The RSS based distance estimation strongly depends on different parameters such as characteristics of the underwater optical channel, divergence angle of the transmitter, field of view of the receiver, transmitted power, and trajectory angle. For a LoS link and achievable data rate $R_{i}^{j}$, the estimated distance $\hat{d}_{i j}$ between node $i$ and $j$ is obtained in [250] from (9) as

$$
\hat{d}_{i j}=\frac{2 \cos \varphi_{i}^{j}}{c(\lambda)} W_{0}\left(\frac{c(\lambda)}{2 \cos \varphi_{i}^{j} \sqrt{\frac{2 \pi T \hbar c R_{i}^{j} r_{j}\left(1-\cos \theta_{i}\right)}{\eta_{t}^{j} \lambda P_{t_{i}} \eta_{t}^{i} \eta_{j} A_{j} \cos \varphi_{i}^{j}}}}\right),
$$

where $T$ is the pulse duration and $W_{0}(\cdot)$ is the real part of Lambert $W$ function [306]. Table 8 summarizes the literature on different ranging techniques for UWONs.

\subsubsection{Localization Techniques for UOWNs}

We divide the localization schemes for UOWNs into two categories as distributed and centralized schemes. In distributed localization schemes, every underwater optical sensor node localizes itself by communicating with multiple anchor nodes. In centralized localization schemes, the underwater optical sensor nodes do not localize themselves but the location information is sent to them by the surface buoy or sink node periodically.

Distributed Localization Schemes for UOWNs. In this section, we summarize two distributed UOWNs localization schemes where one is based on ToA ranging and the other is based on RSS based ranging.

- ToA Based Scheme: In [297], the authors have proposed for the first time a ToA based underwater optical wireless positioning system. The authors considered an OBS placed in an underwater hexagonal cell and a number of users with transceivers capable of UOWC. Each OBS consists of 60 green LEDs forming an underwater OCDMA network where the modulation scheme of OOK is considered. For the ToA scheme, first, the distance is estimated between the users and the OBS by using the relationship of the transmission time of an optical signal, speed, and the distance. It is assumed that all the OBSs and the users are synchronized, and all the OBSs transmit the beacon signals at $\tau=\tau_{0}$. The users receive multiple beacon signals from multiple OBSs at different times namely $\tau_{1}, \tau_{2} \tau_{, \ldots,} \tau_{m}$, where $m$ are the number of OBSs. Different underwater channel impairments such as turbulence, current, and multipath lead to the distance estimation error for ToA ranging. Once the ToA based estimated distances are available from at least three OBSs, the user was able to locate itself in two dimensions by using linear least square solution.

- RSS based Scheme: As the optical signal from the OBSs to the user passes through the underwater environment it suffers from attenuation, absorption, and scattering. The underwater user requires the RSS signals from at least three OBSs in this case as well. RSS scheme has low cost because every transceiver is able to estimate the received signal power. However, the RSS based distance estimation requires precise modeling of the channel [307, 143]. The RSS based distance estimation strongly depends on different parameters such as characteristics of the underwater optical channel, divergence angle of the transmitter, field of view of the receiver, transmitted power, and trajectory angle. The widening and attenuation of the underwater optical signals are dependent on the wavelength. Monte Carlo simulations were used in [297] to find out the RSS based distances. Once the RSS based distances were estimated to at least three OBSs, the user was able to locate itself in two dimensions by using linear least square solution. 
Table 8: Comparison of different ranging techniques for underwater localization.

\begin{tabular}{|l|l|l|l|l|}
\hline \hline Literature & Channel model & Ranging Technique & Accuracy & Complexity \\
\hline$[283,284,285,286]$ & Acoustic & ToA & High & Moderate \\
{$[287,288,289,290,291,292,293]$} & Acoustic & TDoA & High & High \\
{$[294,295,296]$} & Acoustic & RSS & Low & Low \\
{$[297]$} & Optical & ToA & High & Moderate \\
{$[297]$} & Optical & RSS & Low & Low \\
\hline \hline
\end{tabular}
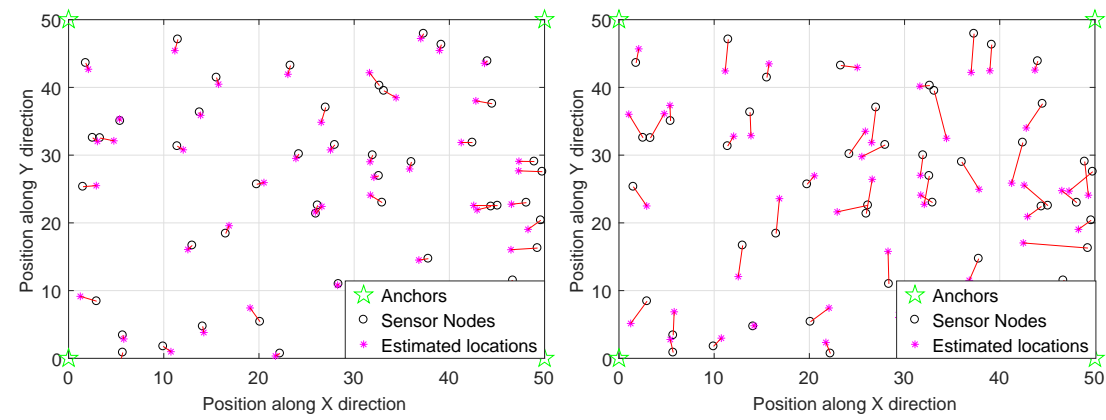

(a) Localization performance of ToA based (b) Localization performance of RSS based distributed UOWNs [297]

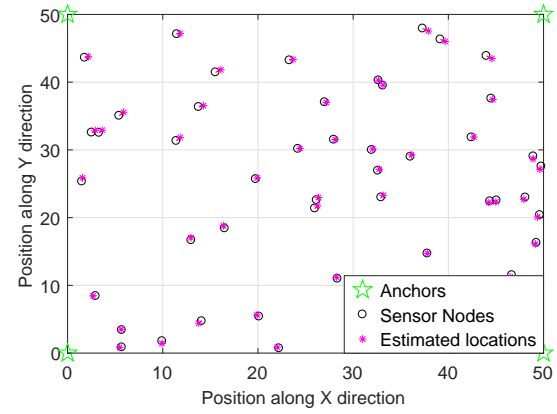
distributed UOWNs [297]

(c) Localization performance of ToA based (d) Localization performance of RSS based centralized UOWNs [272, 184]

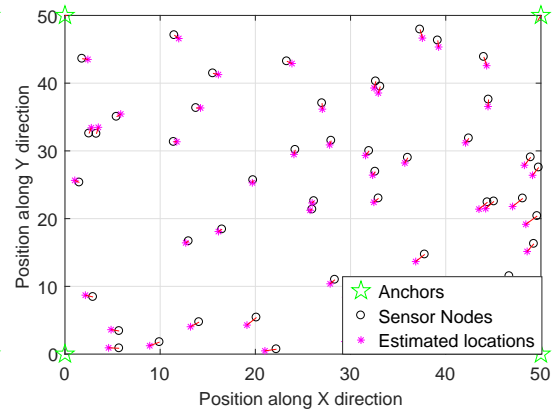
centralized UOWNs [272, 184]

Figure 22: Localization performance of ToA and RSS based distributed and centralized UOWNs.

Table 9: Comparison of different UOWNs localization schemes.

\begin{tabular}{|l|l|l|l|}
\hline \hline Scheme & Method & Computation & Architecture \\
\hline Underwater optical positioning systems [297] & ToA & Distributed & Optical \\
Underwater optical positioning systems [297] & RSS & Distributed & Optical \\
UOWNs localization with limited connectivity [272, 308, 309] & RSS & Centralized & Optical \\
Energy harvesting empowered UOWNs localization [184] & RSS & Centralized & Optical \\
Energy harvesting hybrid acoustic/optical UOWNs localization [45] & RSS & Centralized & Hybrid acoustic/optical \\
\hline \hline
\end{tabular}

Centralized Localization Schemes for UOWNs. In centralized UOWNs localization schemes, the underwater user does not localize itself, but the location is sent to the user by the surface buoy or sink node periodically. To the best of our knowledge, only RSS based centralized localization schemes exist for UOWNs. In [272, 308], we have proposed a localization scheme for UOWNs with limited connectivity. As the transmission range of users in UOWNs is limited, a multihop UOWN was considered and the single neighborhood distances were computed by using RSS. Using these single neighborhood distances a novel distance completion strategy was used by the surface station to get the global view of the whole network. In [184], we presented an energy harvesting empowered under- water optical localization scheme where the underwater sensor nodes were able to harvest the energy from ambient marine sources. As the nodes can collect energy from the underwater environment, it helps to increase the localization accuracy and lifetime of the network. Based on the harvested energy availability, the sensor nodes communicate with its neighbor nodes and computes the RSS ranges. A closed form localization technique was developed to find the location of every optical sensor node in UOWNs. The proposed localization technique accurately minimizes the error function by partitioning the kernel matrix into smaller block matrices. Furthermore, a novel matrix completion strategy was introduced to complete the missing elements in block matrices, which results in better approx- 
imation. In [45], we proposed an energy harvesting localization scheme for hybrid acoustic and optical underwater wireless communications system. A weighting strategy was used in [45] to give more preference to accurate measurements. Recently, in [308] a three-dimensional localization scheme was proposed for UOWNs which accounts for the outliers present in the distance estimation. Moreover, optimal placement of anchors was considered in [309] to improve the accuracy.

\subsubsection{Comparison of Localization Schemes for UOWNs}

In order to compare the different localization schemes for UOWNs, we have simulated two different scenarios where the actual locations of the sensor nodes and anchor nodes are kept same in both scenarios for fair comparison. To evaluate the performance of distributed ToA and RSS based UWONs localization schemes proposed in [297], we considered 50 optical sensor nodes deployed randomly in $50 \mathrm{~m} \times 50 \mathrm{~m}$ square area and 4 anchor nodes deployed at each corner of the area. The optical sensor nodes are able to communicate with at least three anchor nodes directly and localize itself using linear least square solution. Fig. 22a and Fig. 22b shows the localization performance of the two schemes with root mean square error of 0.8 $\mathrm{m}$ and $1.6 \mathrm{~m}$ respectively. To evaluate the performance of centralized ToA and RSS based UWONs localization schemes, we have considered the same scenario of 50 optical sensor nodes deployed randomly in $50 m \times 50 m$ square area and 4 anchor nodes deployed at each corner of the area. But here the limited transmission range of optical sensor nodes is taken into account which leads to multi-hop UOWNs. In this case, the internode single hop distances are measured by the optical sensor nodes and sent to the surface station via surface buoys. The surface station then finds out the location of each optical sensor node by using dimensionality reduction techniques and linear transformations [272, 184]. Fig. 22c and Fig. 22d shows the localization performance of the two schemes with root mean square error of $0.3 \mathrm{~m}$ and $0.9 \mathrm{~m}$ respectively. Table 9 summarizes the UOWNs localization schemes.

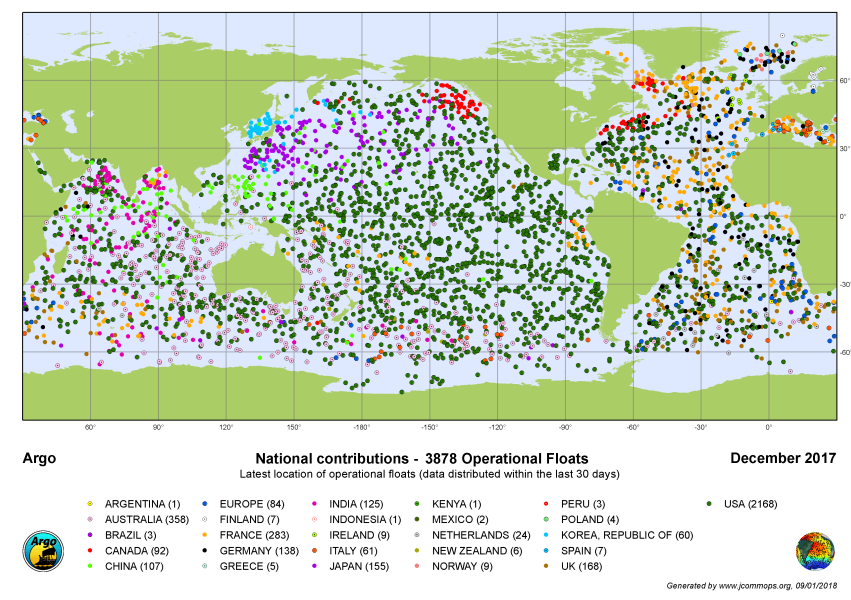

Figure 23: Distribution of floating sensors in Argo [310].

\subsection{State of the Art Underwater Monitoring and Localization Systems}

In the past various techniques were used by the oceanographers for ocean's exploration. The most common monitoring equipment include ocean floor sensors, floating sensors, surface buoys and surface stations. Sensed data from the sensors on the ocean floor is collected by the surface buoys. The surface buoys are fixed and they can send the collected data to the surface station using wired or wireless communications. In case of floating sensors, the sensors do not have fixed location and drift with ocean currents. Floating sensors are dynamic in nature and they can sense a reverberant underwater environment. At present, the largest ocean monitoring system is developed by Global Ocean Data Assimilation Experiment (GODAE) and Oceanview called Argo [310, 311]. Argo consists of 3800 free drifting floating sensors which measure the salinity, currents, and temperature of the ocean up to $2000 \mathrm{~m}$ of depth. The location of Argo float is determined using GPS once it is on the surface of the ocean and it also transmits the data to the onshore station using satellite communication. In Argo project, the floats do not interact with each other and work independently. Fig. 23 shows the current distribution of Argo floats in oceans all over the world. China has announced recently a similar project to Argo, to build underwater monitoring systems across the south and east China seas for intruder detection [312]. In 1980, the U.S. Navy developed a large scale network of underwater devices called Seaweb [313]. Seaweb consisted of AUVs, surface buoys, gliders, repeaters, and surface stations. Seaweb used acoustic waves for underwater communication and RF waves for terrestrial communication.

Acoustic localization systems for underwater monitoring utilizes two different approaches, namely long baseline (LBL) and short baseline (SBL) [314]. In the LBL approach, the acoustic transponders are installed in the underwater operation area. Sensor nodes that are in the coverage of these acoustic transponders respond by using a certain ranging method and localize itself either by using triangulation or trilateration [315]. In the SBL approach, the surface station follows the underwater sensor nodes and transmits short-range acoustic signals for the sensor nodes to localize itself. The SBL underwater positioning systems have been used by Woods Hole Oceanographic Institution to find out the location of a deep underwater ROV [316]. In addition to the LBL and SBL approaches, there exists an underwater localization system called GPS intelligent buoy (GIB). The GIB is a commercial system in which the surface buoy acts as a relay between the surface station and the seabed sensors. GIB collects the distance estimation from the sensors to itself and sends it to a central station where the central station finds the global view of all the seabed sensors. GIB systems have numerous applications which include weapon testing and training [317], tracking AUVs [318], global view of the network [318], and intruder detection [319]. Table 10 summarizes some of the well known commercial underwater localization systems.

\subsection{Summary and Insights}

In the first part of the section, we have introduced the fundamentals and challenges of underwater localization including 
Table 10: Comparison of different commercial underwater localization systems.

\begin{tabular}{|c|c|c|c|c|c|}
\hline System & Company & $\begin{array}{l}\text { Channel } \\
\text { type }\end{array}$ & Approach & Accuracy & Applications \\
\hline $\begin{array}{l}\text { Underwater acoustic LBL positioning } \\
\text { [320]. }\end{array}$ & Evo Logics & Acoustic & LBL & $1.5 \mathrm{~cm}$ & $\begin{array}{l}\text { Offshore positioning, } \\
\text { navigation, cartography, } \\
\text { geodesy, and sensors } \\
\text { tracking. }\end{array}$ \\
\hline $\begin{array}{l}\text { HiPAP - Acoustic underwater position- } \\
\text { ing and navigation systems [321]. }\end{array}$ & KONGSBERG & Acoustic & SBL & $2 \mathrm{~cm}$ & $\begin{array}{l}\text { Seabed positioning of ves- } \\
\text { sels, sub-sea meteorology, } \\
\text { and telemetry. }\end{array}$ \\
\hline $\begin{array}{l}\text { Mini-Ranger } 2 \text { Underwater Positioning } \\
\text { (USBL) system [322]. }\end{array}$ & Sonardyne & Acoustic & Ultra SBL & $2 \mathrm{~cm}$ & $\begin{array}{l}\text { Oil and gas exploration, } \\
\text { marine robotics, and ma- } \\
\text { rine security. }\end{array}$ \\
\hline USBL positioning systems [323] & iXblue & Acoustic & Ultra SBL & $6 \mathrm{~cm}$ & $\begin{array}{l}\text { Hydrography, maritime } \\
\text { vessels, ocean science, } \\
\text { and defense. }\end{array}$ \\
\hline $\begin{array}{l}\text { VideoRay ROV Positioning Systems } \\
\text { [324] }\end{array}$ & $\begin{array}{l}\text { KCF Technolo- } \\
\text { gies }\end{array}$ & Acoustic & Ultra SBL & $150 \mathrm{~cm}$ & $\begin{array}{l}\text { Navigation, tracking, } \\
\text { search and rescue, and } \\
\text { target detection. }\end{array}$ \\
\hline $\begin{array}{l}\text { TrackLink Acoustic Tracking Systems } \\
\text { [325] }\end{array}$ & LinkQuest Inc & Acoustic & Ultra SBL & $0.5 \mathrm{~cm}$ & $\begin{array}{l}\text { Navigation, tracking, un- } \\
\text { derwater surveys, and oil } \\
\text { and gas exploration. }\end{array}$ \\
\hline $\begin{array}{l}\text { Teledyne Benthos underwater acoustic } \\
\text { systems [326] }\end{array}$ & Teledyne Marine & Acoustic & $\begin{array}{l}\text { LBL/Ultra } \\
\text { SBL }\end{array}$ & $5 \mathrm{~cm}$ & $\begin{array}{l}\text { Navigation, tracking, un- } \\
\text { derwater surveys, and oil } \\
\text { and gas exploration. }\end{array}$ \\
\hline
\end{tabular}

but not limited to the node deployment, mobility, hostile underwater channel, and synchronization. The literature on underwater acoustic based ranging is more mature and considers all of the ranging methods while the literature on underwater optical ranging is at an early stage of development and consists of only ToA and RSS based techniques. The localization accuracy of ToA and TDoA based ranging methods is high at the expense of synchronization costs whereas the RSS based ranging provide coarse localization but does not require extra hardware.

The second part of this section covers the localization techniques used for UOWNs. The localization techniques for UOWNs are broadly categorized into distributed and centralized schemes. ToA and RSS based optical ranging methods are compared for distributed and centralized schemes where the localization accuracy of ToA is better than the RSS based methods because the received power in RSS based optical ranging is highly affected by the hostile underwater aquatic channel. In Table 9, we have summarized the localization techniques used for UOWNs.

In the final part, the state of the art underwater monitoring and localization systems (such as Argo and Seaweb, etc.) are briefly discussed. We have also discussed different baseline approaches such as SBL, LBL, and GIB used by the current underwater monitoring and localization systems. In the SBL approach, the sensor nodes or AUVs localizes itself with respect to the surface ship. The limitation of the SBL approach is that the localization accuracy depends on the size of the baseline. In the LBL and GIB approaches, the sensor nodes or AUVs localizes itself by triangulating the acoustic signals from the surface beacons. The limitation of LBL and GIB approaches is the extra cost and time to set up the network. In Table 10, we have summarized some of the well known commercial underwater localization and monitoring systems.

\section{Future Perspectives of UOWNs}

In the following, we will advise some potential future UOWNs research directions.

\subsection{UOWC Channel Modeling}

To model the UOWC channel, there is still a need to further investigate and analyze new theoretical models which can either be developed analytically or computationally. The analytic models for UOWC channel are quite simple because of simplifying the complex nature of photon propagation, but these models are either analytically intractable or hard to evaluate computationally. On the other hand, computational models are complex and their time complexity may not be suitable to employ on a large scale network. Therefore, modeling and performance analysis of UOWNs necessitates accurate and simple UOWC channel models as they are building blocks of UOWNs. RTE can be used as a starting point for Monte Carlo Simulations in 2D or limited 3D scenarios where the simulations can be run in parallel on modern computer architectures.

\subsection{Novel Network Protocols}

The current research on UOWNs is highly concentrated on physical layer problems, which tines out toward the higher layers. To the best of author's knowledge, the networking aspects are studied only in few papers so far [327, 250, 144, 143, 110, $328,137,297,45,272,251,329,330,331]$. Noting that the limited communication range and directivity of UOWCs yield limited network connectivity, implementing UOWNs in real life necessitates adequate protocols and network architectures.

First and foremost, UOWNs requires effective routing algorithms in order to increase the network connectivity and performance by extending the communication range and expanding the coverage. Even though some of the potential routing 
protocols are highlighted in Section 4.3, there are no sufficient efforts toward UOWN routing techniques except [137] which only considers a centralized routing scenario to show the impact of multihop communication on network performance. Therefore, it is quite of interest to develop distributed and dynamic routing algorithms which adapt itself according to environmental and network changes. Furthermore, novel transport layer protocols are required because UOWC channels are quite different from terrestrial and underwater acoustic wireless networks.

\subsection{Cross Layer Design Issues}

Even though we have surveyed the UOWNs following a strictly layered perspective, which is traditionally employed for wired networks, considering a cross-layer design could improve the overall system performance to a great extent. Indeed, QoS requirements of the application layer can only be satisfied by mapping them into the lower-layer metrics such as end-to-end data rate, delay, energy efficiency, packet loss, etc. Accordingly, it is interesting to investigate a cross-layer optimization framework which adapts physical layer parameters (e.g., divergence angle, transmission power, communication range, etc.) to channel conditions and dynamically change the routing paths to satisfy QoS requirements, avoid congestion, increase the reliability, and maintain the network connectivity.

\subsection{Localization in UOWNS}

Localization is of utmost importance for UOWNs where it can be used for node tracking, intruder detection, and data tagging. A greater number of underwater applications demands distributed localization schemes as they can provide online monitoring. However, few research works are carried out to develop distributed [297] and centralized [272, 45, 184, 309] localization schemes for UOWNs. Due to the severe UOWC channel conditions distributed localization schemes for UOWNs are challenging and needs further investigation. Limited range of UOWC links and higher energy consumption of distributed schemes led to the development of centralized UOWNs localization schemes where the localization is performed at the surface station. Centralized localization schemes are good to get the overall global view of the UOWN. Moreover, the impact of localization schemes on location-based routing and clustering for UOWNs still need to be investigated. Also, the cross-layer schemes such as the impact of link quality, connectivity, transmission range, and energy on localization performance are open issues.

\subsection{Practical Implementations of UOWNs}

Research on implementation of UOWNs is limited and further need to be studied. There is a dire need to develop underwater optical transceivers which can overcome the problem of link misalignment, low transmission range, low bandwidth, energy consumption, and compactness. There is a great potential to develop more advanced low cost and low power underwater transmission light sources, receiving nodes, and energy harvesting systems. Testing of the UOWNs also needs to be carried out in the real underwater environment. Hybrid systems comprising of both acoustic and optical underwater wireless communication system have been introduced in [42, 43, 44]. Authors in [332] have explored a statistical analogy between underwater acoustic and optical wireless links for predicting the signal to noise ratio of underwater optical links. The research on developing hybrid systems is still in its infancy and needs proper analysis. Also, the adaptive switching between an acoustic and optical mode for different operations need extra attention.

\subsection{Energy Harvesting for UOWNs}

Underwater optical sensor nodes have limited energy resources, which has a substantial impact on the network lifetime. Taking the engineering hardship and monetary cost of battery replacement into account, an energy self-sufficient UOWN is essential to maximize the network lifetime. In this regard, energy harvesting is a promising solution to collect energy from the ambient sources in the aquatic environment and storing it in an energy buffer. As surveyed in [239], ongoing research efforts on terrestrial communications have shown that energy harvesting plays a significant role in enhancing performance. However, most of the energy harvesting techniques are designed for outdoor environments and are not applicable to the aquatic environment. Recently, acoustics resonators are used in [333] to acquire acoustic energy from the underwater environment and harvest to the sensor nodes. A muti-source energy harvesting system was proposed in [45, 184] which harvests energy from multiple underwater sources such as acoustic resonators and MFCs [240], and harvested to the sensor nodes. Moreover, albeit the notable research body on designing different protocols for underwater communication networks, no significant research is carried out on the energy harvesting methods for UOWNs.

\subsection{Towards Internet of Underwater Things (IoUTs)}

There has recently been a growing interest in developing internet of underwater things (IoUTs) which can lead to enabling various underwater applications [334]. In the recent past, several attempts have been made to develop routing [335, 336], scheduling [337], and data analytic [338] techniques for IoUTs. The IoUTs research is still in its infancy and needs to be more explored. Multi-hop UOWNs can be a potential technology to implement the IoUTs because of its low power consumption and higher data rate.

\subsection{Full-Duplex Communication for UOWNs}

Today's UWC systems generally utilize the half-duplex links for communication. One of the main reason behind this limitation is the challenging underwater environment for any type of wireless communication channel. The effects of attenuation, absorption, scattering, refraction, salinity, temperature, and pressure effects the propagation of waves in an underwater environment. Due to the aforementioned reasons, most of the UWC systems are still half-duplex and use the same frequency for transmission and reception of the data. Recently, authors of [339] have introduced an in-band full duplex communication 
mode for UANs which still needs to develop self-interference cancellation mechanisms, cross-layer design, and mitigation of multipath effect. Few studies exist on the full duplex mode of operation for UOWC systems. For instance, a hybrid acousticoptical full duplex communication system has been proposed in [43] where the downlink from AUV to the base station is based on acoustic waves while for the uplink, directional optical communication is used to achieve higher data rates. Regardless of the communication medium, to the best of our knowledge, there is no full duplex optical wireless communication implementation yet. The research work on developing the full-duplex communication mode for UOWC is in its earlier stage and stays as an interesting open research problem to be investigated.

\subsection{Software Defined Networks (SDN) for UOWNs}

The existing architectures of UWC systems are inflexible and are mainly based on hardware, which poses a significant challenge to adopt new underwater technologies. Fortunately, software-defined network (SDN) is an emerging paradigm to overcome this problem by separating the data and control planes and managing the network via an SDN controller. In [340], the authors have presented a comprehensive survey on SDN for optical networks which is very useful to implement SDN for UOWC systems. In [341], the concept of employing SDN for underwater communication systems was introduced (SoftWater). The architecture of SoftWater consists of underwater sensors, AUVs, surface buoys, ships, and in/out-band control channels. The sensors and AUVs in SoftWater system can transmit the sensing data to the surface buoys by using hybrid links such as acoustic, optical, and magnetic induction (MI) [342]. The surface buoys and ships act as a sink to collect the sensed data and forward it to the on-ground station. Additionally, the surface buoys and ships also perform the network management task. In-band channels are established by using optical or MI links for the communication between the underwater devices to provide high bandwidth, short range, and low delay links while out-band channels are established by using acoustic links (long range, high delay, and low bandwidth) between the underwater devices and the surface buoy/ship. However, the implementation of SoftWater suffers from numerous challenges such as the 3D deployment of the SDN controller, mobility aware traffic balancing, efficient network hypervisor, and wireless channel hypervisor. These issues are still open research problems and need to be investigated in the future for the SDN based UOWC systems.

\subsection{Magnetic Induction for UOWC}

In recent years, the research community on magnetic induction (MI) based communications have demonstrated that MI is a good option for communication in harsh environments such as water pipelines, soil and oil reservoirs [343, 344]. Therefore, mathematical analysis has been provided in [345, 346, 347] to use MI for underwater communications. MI-based underwater communication can provide low propagation delay, predictable channel response, large communication range, high data rate, and stealth mode of operation [348]. However, the research on
MI-based underwater communications is in its early stage and need to be investigated further in the future.

\section{Conclusions}

In this paper, we have presented a comprehensive survey of underwater optical wireless networks (UOWNs) research. This survey covers different aspects of cutting-edge UOWNs from a layer by layer perspective. Firstly, each layer of UOWNs such as physical, data link, networking, transport, and application layers are briefly presented and then localization techniques for UOWNs are surveyed. We started with defining different possible architectures for UOWNs and then the issues related to each layer are thoroughly discussed. Besides providing the technical background on UOWNs, we have also provided details on the challenges to design a practical UOWN. Additionally, localization is an important task where the location of the underwater optical sensor node can be used for node tracking, intruder detection, and data tagging. Conventional terrestrial and underwater acoustic localization schemes do not meet the requirements of UOWNs where the unfavorable behavior of UOWC asks for novel localization schemes. Even though we have surveyed the state of the art localization schemes for UOWNs, the subject remains open and requires to develop accurate and practical localization schemes. To reach this goal, communication, networking, and localization in UOWNs require more research efforts. In short, this survey can help the novice readers to get an insight into each layer and localization of UOWNs which can lead to the development of practical UOWNs.

\section{References}

[1] NOAA, How much water is in the ocean?, https://oceanservice. noaa.gov/facts/oceanwater.html, online; accessed 10 Dec. 2017.

[2] C. W. Thomson, J. Murray, G. S. Nares, F. T. Thomson, Report on the scientific results of the voyage of HMS Challenger during the years 1873-76 under the command of Captain George S. Nares and the late Captain Frank Tourle Thomson, RN, Vol. 29, Printed for HM Stationery off., 1888.

[3] A. F. Laloe, The Geography of the Ocean: Knowing the ocean as a space (Studies in Historical Geography), Routledge, Howick Place, London, 2016.

[4] O. Kruemmel, Handbuch der Ozeanographie, Europaischer Hochschulverlag Gmbh, Bremen, Germany, 2014.

[5] J. H. and Journal S. Murray and Journal Journal Adolf, The Depths of the Oceans, Wentworth Press, New Hampshire, US, 2016.

[6] E. J. P. and S. A. Earle, The Oceans, McGraw-Hill, New York, US, 2001.

[7] M. N. Hill, The Sea, Harvard University Press, Cambridge, US, 2001.

[8] R. W. Fairbridge, The encyclopedia of oceanography, Reinhold Pub., New York, US, 1966.

[9] M. Stojanovic, Recent advances in high-speed underwater acoustic communications, IEEE J. Ocean. Eng. 21 (2) (1996) 125-136.

[10] A. Zielinski, Y. H. Yoon, L. Wu, Performance analysis of digital acoustic communication in a shallow water channel, IEEE J. Ocean. Eng. 20 (4) (1995) 293-299.

[11] H. Ochi, Y. Watanabe, T. Shimura, Basic Study of Underwater Acoustic Communication Using 32-Quadrature Amplitude Modulation, Japanese J. of App. Physics 44 (6S) (2005) 4689.

[12] H. C. Song, W. S. Hodgkiss, Efficient use of bandwidth for underwater acoustic communication, The J. of the Acous. Soc. of Am. 134 (2) (2013) 905-908.

[13] DesertStar, Desert star systems, http://desertstar.com/product/ sam-1/, online; accessed 02 Feb. 2019. 
[14] TriTech, Tritech micron modem features, http://www.tritech.co. uk/product/micron-data-modem, online; accessed 02 Feb. 2019.

[15] LinkQuest, Linkquest underwater acoustic modems features, http: //link-quest.com/html/models1.htm, online; accessed 02 Feb. 2019.

[16] EvoLogics, Evologics acoustic modems features., http://www . evologics.de/en/products/acoustics/index.html, online; accessed 02 Feb. 2019

[17] Teledyne, Features of acoustic modems teledyne benthos, http:// www.f-e-t.com/images/uploads/Teledyne_Benthos, online; accessed 02 Feb. 2019

[18] Oceania, GPM 3000 acoustic modem features., http: //www2.1-3com.com/oceania//pdfs/datasheets/GPM $\% 20300 \%$ 20 Acoustic $\% 20$ Modem $\% 20$ Spec $\% 2$ Sheet $\% 20$ Rev $\% 201 \% 206$.pdf, online; accessed 02 Feb. 2019.

[19] R. K. Moore, Radio communication in the sea, IEEE Spectrum 4 (11) (1967) 42-51.

[20] A. I. Al-Shamma'a, A. Shaw, S. Saman, Propagation of electromagnetic waves at $\mathrm{MHz}$ frequencies through seawater, IEEE Trans. Antennas Propag. 52 (11) (2004) 2843-2849.

[21] A. Shaw, A. I. Al-Shamma'a, S. R. Wylie, D. Toal, Experimental investigations of electromagnetic wave propagation in seawater, in: European Microwave Conf., 2006, pp. 572-575.

[22] C. Uribe, W. Grote, Radio Communication Model for Underwater WSN, in: 3rd Int. Conf. on New Technologies, Mobility and Security, 2009, pp. $1-5$.

[23] Z. Zeng, S. Fu, H. Zhang, Y. Dong, J. Cheng, A survey of underwater optical wireless communications, IEEE Commun. Surveys Tuts. 19 (1) (2016) 204-238.

[24] S. Q. Duntley, Light in the sea*, J. Opt. Soc. Am. 53 (2) (1963) 214-233.

[25] G. D. Gilbert, T. R. Stoner, J. L. Jernigan, Underwater experiments on the polarization, coherence, and scattering properties of a pulsed bluegreen laser, Proc. SPIE 0007 (1966) 07 - 14.

[26] F. Hanson, S. Radic, High bandwidth underwater optical communication, Appl. Opt. 47 (2) (2008) 277-283.

[27] S. Arnon, Underwater optical wireless communication network, Opt. Engineering 49 (6) (2010) 1-15.

[28] M. Doniec, M. Angermann, D. Rus, An end-to-end signal strength model for underwater optical communications, IEEE J. Ocean. Eng. 38 (4) (2013) 743-757.

[29] J. S. Jaffe, Underwater optical imaging: The past, the present, and the prospects, IEEE J. Ocean. Eng. 40 (3) (2015) 683-700.

[30] J. Xu, M. Kong, A. Lin, Y. Song, X. Yu, F. Qu, J. Han, N. Deng, OFDMbased broadband underwater wireless optical communication system using a compact blue LED, Opt. Commun. 369 (Supplement C) (2016) 100 -105 .

[31] C. Wang, H.-Y. Yu, Y.-J. Zhu, T. Wang, Y.-W. Ji, Multi-LED parallel transmission for long distance underwater VLC system with one SPAD receiver, Opt. Commun. 410 (2017) 889 - 895.

[32] G. Cossu, A. Sturniolo, A. Messa, D. Scaradozzi, E. Ciaramella, FullFledged 10Base-T Ethernet Underwater Optical Wireless Communication System, IEEE J. Sel. Areas Commun. 36 (1) (2018) 194-202.

[33] M. Atef, R. Swoboda, H. Zimmermann, Real-Time 1.25-Gb/s Transmission Over 50-m SI-POF Using a Green Laser Diode, IEEE Photon. Technol. Lett. 24 (15) (2012) 1331-1333.

[34] B. Cochenour, L. Mullen, J. Muth, Temporal response of the underwater optical channel for high-bandwidth wireless laser communications, IEEE J. Ocean. Eng. 38 (4) (2013) 730-742.

[35] D. Tsonev, S. Videv, H. Haas, Towards a $100 \mathrm{~Gb} / \mathrm{s}$ visible light wireless access network, Opt. Express 23 (2) (2015) 1627-1637.

[36] K. Nakamura, I. Mizukoshi, M. Hanawa, Optical wireless transmission of $405 \mathrm{~nm}, 1.45 \mathrm{Gbit} / \mathrm{s}$ optical IM/DD-OFDM signals through a $4.8 \mathrm{~m}$ underwater channel, Opt. Express 23 (2) (2015) 1558-1566.

[37] H. M. Oubei, C. Li, K.-H. Park, T. K. Ng, M.-S. Alouini, B. S. Ooi, 2.3 Gbit/s underwater wireless optical communications using directly modulated 520 nm laser diode, Opt. Express 23 (16) (2015) 20743-20748.

[38] S. P. Najda, P. Perlin, T. Suski, L. Marona, M. Leszczyński, P. Wisniewski, R. Czernecki, R. Kucharski, G. Targowski, M. A. Watson, H. White, S. Watson, A. E. Kelly, AlGaInN laser diode technology for $\mathrm{GHz}$ high-speed visible light communication through plastic optical fiber and water, Opt. Engineering 55 (6) (2016) 55.
[39] H. M. Oubei, J. R. Duran, B. Janjua, H.-Y. Wang, C.-T. Tsai, Y.-C. Chi, T. K. Ng, H.-C. Kuo, J.-H. He, M.-S. Alouini, G.-R. Lin, B. S. Ooi, 4.8 Gbit/s 16-QAM-OFDM transmission based on compact 450-nm laser for underwater wireless optical communication, Opt. Express 23 (18) (2015) 23302-23309.

[40] J. Xu, Y. Song, X. Yu, A. Lin, M. Kong, J. Han, N. Deng, Underwater wireless transmission of high-speed QAM-OFDM signals using a compact red-light laser, Opt. Express 24 (8) (2016) 8097-8109.

[41] T.-C. Wu, Y.-C. Chi, H.-Y. Wang, C.-T. Tsai, G.-R. Lin, Blue laser diode enables underwater communication at $12.4 \mathrm{Gbps}$, Scientific Reports 7 (8) (2017) 40480

[42] S. Han, Y. Noh, R. Liang, R. Chen, Y.-J. Cheng, M. Gerla, Evaluation of underwater optical-acoustic hybrid network, China Commun. 11 (5) (2014) 49-59.

[43] L. J. Johnson, R. J. Green, M. S. Leeson, Hybrid underwater optical/acoustic link design, in: 16th Int. Conf. on Trans. Opt. Netw., (ICTON), 2014, pp. 1-4

[44] C. Moriconi, G. Cupertino, S. Betti, M. Tabacchiera, Hybrid acoustic/optic communications in underwater swarms, in: OCEANS, 2015, pp. 1-9.

[45] N. Saeed, A. Celik, T. Y. Al-Naffouri, M.-S. Alouini, Energy harvesting hybrid acoustic-optical underwater wireless sensor networks localization, Sensors 18 (1) (2017) 1-16.

[46] C. Lodovisi, P. Loreti, L. Bracciale, S. Betti, Performance analysis of hybrid optical-acoustic AUV swarms for marine monitoring, Future Internet 10 (7) (2018) 1-18.

[47] M. G. G. Camila, S. R. D. Paulo, L. R. C. Marcello, A. M. Wallace, M. C. Felipe, N. G. Jonathan, A survey of underwater wireless communication technologies, J. of Commun. and Info. Sys. 31 (1) (2016) 242-255.

[48] H. Kaushal, G. Kaddoum, Underwater optical wireless communication, IEEE Access 4 (2016) 1518-1547.

[49] L. J. Johnson, R. J. Green, M. S. Leeson, Underwater optical wireless communications: depth dependent variations in attenuation, Appl. Opt. 52 (33) (2013) 7867-7873.

[50] L. J. Johnson, F. Jasman, R. Green, M. S. Leeson, Recent advances in underwater optical wireless communications, Underwater Techno. 32 (3) (2014) 167-175

[51] L. K. Gkoura, G. D. Roumelas, H. E. Nistazakis, H. G. Sandalidis, A. Vavoulas, A. D. Tsigopoulos, G. S. Tombras, Underwater optical wireless communication sys.: A concise review, in: Turbulence Modelling Approaches - Current State, Development Prospects, Applications, InTech, 2017.

[52] M. M. Alomari, M. Wafa, A. Rehab, G. G. Babhair, M. Hemalatha, Vision and challenges of underwater optical wireless communication - A survey, Int. J. of Computer Applications 167 (8) (2017) 8-10.

[53] B. Truax, Acoustic Communication, Ablex, Westport, CT, USA, 2001.

[54] E. M. Sozer, M. Stojanovic, J. G. Proakis, Underwater acoustic networks, IEEE J. Ocean. Eng. 25 (1) (2000) 72-83.

[55] D. Pompili, I. F. Akyildiz, Overview of networking protocols for underwater wireless communications, IEEE Commun. Mag. 47 (1) (2009) 97-102.

[56] I. F. Akyildiz, D. Pompili, T. Melodia, Underwater acoustic sensor networks: research challenges, Ad Hoc Netw. 3 (3) (2005) 257 - 279.

[57] W. W. Au, P. E. Nachtigall, J. L. Pawloski, Acoustic effects of the ATOC signal $(75 \mathrm{~Hz}, 195 \mathrm{~dB})$ on dolphins and whales, The J. of the Acous. Soc. of Am. 101 (5) (1997) 2973-2977.

[58] C. A. Altgelt, The world's largest radio station, https://www.hep. wisc. edu/ prepost/ELF.pdf, online; accessed 19 Dec. 2017.

[59] M. R. Frater, M. J. Ryan, R. M. Dunbar, Electromagnetic communications within swarms of autonomous underwater vehicles, in: Proc. of the 1st ACM Int. Workshop on Underwater Networks, 2006, pp. 64-70.

[60] P. Da, Evaluation of wi-fi underwater networks in freshwater, Ph.D. thesis, Ph. D. Thesis, Universidade Do Porto, Porto, Portugal (2014).

[61] V. I. Haltrin, Two-term Henyey-Greenstein light scattering phase function for seawater, in: Proc. of IEEE Int. Symp. on Geoscience and Remote Sensing, Vol. 2, 1999, pp. 1423-1425.

[62] V. I. Haltrin, One-parameter two-term Henyey-Greenstein phase function for light scattering in seawater, Appl. Opt. 41 (6) (2002) 1022-1028.

[63] D. Toublanc, Henyey-Greenstein and Mie phase functions in Monte Carlo radiative transfer computations, Appl. Opt. 35 (18) (1996) 32703274 . 
[64] C. Machado, L. Freitag, A. Bowen, N. E. Farr, Systems and methods for establishing an underwater optical communication network, US Patent 8,953,944 (Feb 2015)

[65] Y. Dong, S. Tang, X. Zhang, Effect of random sea surface on downlink underwater wireless optical communications, IEEE Commun. Lett. 17 (11) (2013) 2164-2167.

[66] H. Zhang, Y. Dong, Link misalignment for underwater wireless optical communications, in: Adva. in Wireless and Opt. Commun., (RTUWO), 2015, pp. 215-218

[67] X. Yi, Z. Li, Z. Liu, Underwater optical communication performance for laser beam propagation through weak oceanic turbulence, Appl. Opt 54 (6) (2015) 1273-1278.

[68] M. Chaplin, Water absorption spectrum, http://www1.1sbu.ac.uk/ water/water_vibrational_spectrum.html, online; accessed 10 Dec. 2017.

[69] J. R. V. Zaneveld, Light and water: Radiative transfer in natural waters (1995).

[70] R. W. Spinrad, K. L. Carder, M. J. Perry, Ocean optics, Vol. 25, Oxford University Press, 1994

[71] C. Mobley, E. Boss, C. Roesler, Ocean optics web book (2010).

[72] C. Gabriel, M. A. Khalighi, S. Bourennane, P. Léon, V. Rigaud, Misalignment considerations in point-to-point underwater wireless optical links, in: OCEANS, 2013, pp. 1-5.

[73] S. K. Sahu, P. Shanmugam, A theoretical study on the impact of particle scattering on the channel characteristics of underwater optical communication system, Optics Commun. 408 (2018) 3 - 14.

[74] C. Mobley, Light and Water: Radiative Transfer in Natural Waters, Academic Press, 1994

[75] T. J. Petzold, Volume scattering functions for selected ocean waters, Tech. rep., Scripps Institution of Oceanography, San Diego, Calif. (1972).

[76] R. C. Smith, K. S. Baker, Optical properties of the clearest natural waters (200-800 nm), Appl. Opt. 20 (2) (1981) 177-184.

[77] J. H. Smart, Underwater optical communications Systems part 1: variability of water optical parameters, in: IEEE Military Commun. Conf., (MILCOM), 2005, pp. 1140-1146 Vol. 2.

[78] J. W. Giles, I. N. Bankman, Underwater optical communications sys part 2: basic design considerations, in: IEEE Military Commun. Conf., (MILCOM), 2005, pp. 1700-1705.

[79] J. R. Apel, Edited by, in: J. R. Apel (Ed.), Principles of Ocean Physics, Vol. 38 of Int. Geophysics, Academic Press, 1988, pp. $1-634$.

[80] A. L. Alldredge, M. W. Silver, Characteristics, dynamics and significance of marine snow, Progress in Oceanography 20 (1) (1988) 41-82.

[81] B. M. Cochenour, L. J. Mullen, A. E. Laux, Characterization of the beam-spread function for underwater wireless optical communications links, IEEE J. Ocean. Eng. 33 (4) (2008) 513-521.

[82] H. V. D. Hulst, Multiple Light Scattering, Academic Press, 1980.

[83] C. Li, K. H. Park, M. S. Alouini, On the use of a direct radiative transfer equation solver for path loss calculation in underwater optical wireless channels, IEEE Wireless Commun. Lett. 4 (5) (2015) 561-564.

[84] S. Arnon, J. Barry, G. Karagiannidis, R. Schober, M. Uysal, Advanced Optical Wireless Communication Sys., 1st Edition, Cambridge University Press, New York, NY, USA, 2012.

[85] S. Jaruwatanadilok, Underwater wireless optical communication channel modeling and performance evaluation using vector radiative transfer theory, IEEE J. Sel. Areas Commun. 26 (9) (2008) 1620-1627.

[86] B. Cochenour, L. Mullen, A. Laux, Spatial and temporal dispersion in high bandwidth underwater laser communication links, in: IEEE Military Commun. Conf., (MILCOM), 2008, pp. 1-7.

[87] J. R. Potter, M. B. Porter, J. C. Preisig, UComms: A Conf. and Workshop on Underwater Commun., Channel Modeling, and Validation, IEEE J. Ocean. Eng. 38 (4) (2013) 603-613.

[88] R. A. Leathers, T. V. Downes, C. O. Davis, C. D. Mobley, Monte carlo radiative transfer simulations for ocean optics: a practical guide, Tech. rep., Naval Research Lab Washington DC Applied Optics Branch (2004).

[89] C. Gabriel, M. A. Khalighi, S. Bourennane, P. Leon, V. Rigaud, Channel modeling for underwater optical communication, in: IEEE GLOBECOM Workshops (GC Wkshps), 2011, pp. 833-837.

[90] J. Li, Y. Ma, Q. Zhou, B. Zhou, H. Wang, Monte carlo study on pulse response of underwater optical channel, Opt. Engineering 51 (2012) 51
$-51-6$.

[91] J. Li, Y. Ma, Q. Zhou, B. Zhou, H. Wang, Channel capacity study of underwater wireless optical communications links based on monte carlo simulation, J. of Opt. 14 (1) (2012) 1-7.

[92] F. Hanson, M. Lasher, Effects of underwater turbulence on laser beam propagation and coupling into single-mode optical fiber, Appl. Opt. 49 (16) (2010) 3224-3230.

[93] W. Liu, Z. Xu, L. Yang, SIMO detection schemes for underwater optical wireless communication under turbulence, Photon. Res. 3 (3) (2015) 48 53.

[94] W. W. Hou, A simple underwater imaging model, Opt. Lett. 34 (17) (2009) 2688-2690.

[95] S. Woods, W. Hou, W. Goode, E. Jarosz, A. Weidemann, Measurements of turbulence for quantifying the impact of turbulence on underwater imaging, in: 2011 IEEE/OES 10th Current, Waves and Turbulence Measurements (CWTM), 2011, pp. 179-183.

[96] M. Holohan, J. Dainty, Low-order adaptive optics: a possible use in underwater imaging?, Opt. \& Laser Techno. 29 (1) (1997) 51 - 55.

[97] V. V. Nikishov, V. I. Nikishov, Spectrum of turbulent fluctuations of the sea-water refraction index, Int. J. of Fluid Mech. Res. 27 (1) (2000) 8298.

[98] O. Korotkova, N. Farwell, Effect of oceanic turbulence on polarization of stochastic beams, Opt. Commun. 284 (7) (2011) 1740-1746.

[99] W. C. Cox Jr, Simulation, modeling, and design of underwater optical communication Sys., North Carolina State University, 2012.

[100] Y. Ata, Y. Baykal, Field correlation of spherical wave in underwater turbulent medium, Appl. Opt. 53 (33) (2014) 7968-7971.

[101] S. Tang, X. Zhang, Y. Dong, Temporal statistics of irradiance in moving turbulent ocean, in: OCEANS, 2013, pp. 1-4

[102] Y. Ata, Y. Baykal, Scintillations of optical plane and spherical waves in underwater turbulence, J. Opt. Soc. Am. A 31 (7) (2014) 1552-1556.

[103] F. Yang, J. Cheng, T. A. Tsiftsis, Free-space optical communication with nonzero boresight pointing errors, IEEE Trans. Commun. 62 (2) (2014) 713-725.

[104] R. Sanchez, N. J. McCormick, Analytic beam spread function for ocean optics applications, Appl. Opt. 41 (30) (2002) 6276-6288.

[105] A. Celik, N. Saeed, B. Shihada, T. Y. Al-Naffouri, M.-S. Alouini, End-to-end performance analysis of underwater optical wireless relaying and routing techniques under location uncertainty, arXiv preprint arXiv:1901.09357.

[106] G. Baiden, Y. Bissiri, High bandwidth optical networking for underwater untethered telerobotic operation, in: OCEANS, 2007, pp. 1-9.

[107] G. Baiden, Y. Bissiri, A. Masoti, Paving the way for a future underwater omni-directional wireless optical communication systems, Ocean Engineering 36 (9) (2009) 633-640.

[108] N. Farr, A. Chave, L. Freitag, J. Preisig, S. White, D. Yoerger, P. Titterton, Optical modem technology for seafloor observatories, in: OCEANS, 2005, pp. 928-934.

[109] S. Tang, Y. Dong, X. Zhang, On link misalignment for underwater wireless optical communications, IEEE Commun. Lett. 16 (10) (2012) 1688 1690.

[110] M. V. Jamali, J. A. Salehi, F. Akhoundi, Performance studies of underwater wireless optical communication systems with spatial diversity: MIMO scheme, IEEE Trans. Commun. 65 (3) (2017) 1176-1192.

[111] B. Cochenour, A. Laux, L. Mullen, Temporal dispersion in underwater laser communication links: Closing the loop between model and experiment, in: IEEE Third Underwater Commun. and Networking Conf., (UComms), 2016, pp. 1-5.

[112] B. Cochenour, K. Dunn, A. Laux, L. Mullen, Experimental measurements of the magnitude and phase response of high-frequency modulated light underwater, Appl. Opt. 56 (14) (2017) 4019-4024.

[113] M. Cole, T. Driscoll, The Lighting Revolution: If We Were Experts Before, We're Novices Now, IEEE Trans. Ind. Appl. 50 (2) (2014) 1509 1520 .

[114] L. Grobe, A. Paraskevopoulos, J. Hilt, D. Schulz, F. Lassak, F. Hartlieb, C. Kottke, V. Jungnickel, K. D. Langer, High-speed visible light communication systems, IEEE Commun. Mag. 51 (12) (2013) 60-66.

[115] H. Haas, L. Yin, Y. Wang, C. Chen, What is LiFi?, J. Lightwave Technol. 34 (6) (2016) 1533-1544.

[116] H. Marshoud, P. C. Sofotasios, S. Muhaidat, G. K. Karagiannidis, Multiuser techniques in visible light communications: A survey, in: Int. Conf. 
on Adv. Commun. Sys. and Info. Sec., (ACOSIS), 2016, pp. 1-6.

[117] M. Jaber, M. A. Imran, R. Tafazolli, A. Tukmanov, 5G Backhaul Challenges and Emerging Research Directions: A Survey, IEEE Access 4 (2016) 1743-1766

[118] T. Ho, S. Trisno, I. I. Smolyaninov, S. D. Milner, C. C. Davis, Studies of pointing, acquisition, and tracking of agile optical wireless transceivers for free-space optical communication networks, in: Proc. SPIE, 2004, pp. 5237-5237.

[119] A. S. Fletcher, S. A. Hamilton, J. D. Moores, Undersea laser communication with narrow beams, IEEE Commun. Mag. 53 (11) (2015) 49-55.

[120] S. Arnon, D. Kedar, Non-line-of-sight underwater optical wireless communication network, J. Opt. Soc. Am. A 26 (3) (2009) 530-539.

[121] J. C. Juarez, A. Dwivedi, A. R. Hammons, S. D. Jones, V. Weerackody, R. A. Nichols, Free space optical communications for next-generation military networks, IEEE Commun. Mag. 44 (11) (2006) 46-51.

[122] D. Liu, X. Zhang, S. Zhang, LED Lens for Rectangular Beam with Small Divergence Angles, J. Opt. Soc. Korea 20 (6) (2016) 739-744.

[123] J. M. Kahn, J. R. Barry, Wireless infrared communications, Proc. of the IEEE 85 (2) (1997) 265-298.

[124] X. Ning, R. Winston, J. O'Gallagher, Dielectric totally internally reflecting concentrators, Appl. Opt. 26 (2) (1987) 300-305.

[125] J. P. Savicki, S. P. Morgan, Hemispherical concentrators and spectral filters for planar sensors in diffuse radiation fields, Appl. Opt. 33 (34) (1994) 8057-8061.

[126] B. Cochenour, L. Mullen, A. Laux, Phase coherent digital communications for wireless optical links in turbid underwater environments, in: OCEANS, 2007, pp. 1-5.

[127] W. C. Cox, B. L. Hughes, J. F. Muth, A polarization shift-keying system for underwater optical communications, in: OCEANS, 2009, pp. 1-4.

[128] J. B. Snow, J. P. Flatley, D. E. Freeman, M. A. Landry, C. E. Lindstrom, J. R. Longacre, J. A. Schwartz, Underwater propagation of high-datarate laser communications pulses, in: Proc. SPIE, Vol. 1750, 1992, pp. $1750-1750-9$

[129] C. Pontbriand, N. Farr, J. Ware, J. Preisig, H. Popenoe, Diffuse highbandwidth optical communications, in: OCEANS, 2008, pp. 1-4.

[130] M. Elamassie, F. Miramirkhani, M. Uysal, Channel modeling and performance characterization of underwater visible light communications, in: IEEE Int. Conf. on Commun. Works., (ICC Workshops), 2018, pp. $1-5$.

[131] M. Elamassie, F. Miramirkhani, M. Uysal, Performance characterization of underwater visible light communication, IEEE Trans. on Commun. 67 (1) (2019) 543-552.

[132] F. Jasman, R. J. Green, Monte Carlo simulation for underwater optical wireless communications, in: 2nd Int. Workshop on Opt. Wireless Commun., (IWOW), 2013, pp. 113-117.

[133] D. Kedar, S. Arnon, Non-line-of-sight optical wireless sensor network operating in multiscattering channel, Appl. Opt. 45 (33) (2006) 8454 8461.

[134] D. Kedar, Multiaccess interference in a non-line-of-sight ultraviolet optical wireless sensor network, Appl. opt. 46 (23) (2007) 5895-5901.

[135] L. Mullen, B. Cochenour, W. Rabinovich, R. Mahon, J. Muth, Backscatter suppression for underwater modulating retroreflector links using polarization discrimination, Appl. Opt. 48 (2) (2009) 328-337.

[136] B. Cochenour, L. Mullen, W. Rabinovich, R. Mahon, Underwater optical communications with a modulating retro-reflector, in: Proc. SPIE, 2009, pp. $1-11$.

[137] A. Celik, N. Saeed, T. Y. Al-Naffouri, M.-S. Alouini, Modeling and performance analysis of multihop underwater optical wireless sensor networks, in: IEEE Wireless Commun. and Netw. Conf., (WCNC), 2018, pp. $1-6$.

[138] P. Eraerds, M. Legre, A. Rochas, H. Zbinden, N. Gisin, SiPM for fast photon-counting and multiphoton detection, Optics Express 15 (22) (2007) 14539-14549.

[139] ITUT, Forward error correction for high bit rate dwdm submarine systems, Tech. rep., International Telecommunication Union (ITU-T) (2004).

[140] M. Bilgi, M. Yuksel, Multi-element Free-Space-Optical spherical structures with intermittent connectivity patterns, in: Proc. IEEE Int. Conf. on Computer Commun. (INFOCOM), 2008, pp. 1-4.

[141] D. E. Ingber, The architecture of life, Scientific American 278 (1) (1998) 48-57.
[142] F. Akhoundi, J. A. Salehi, A. Tashakori, Cellular Underwater Wireless Optical CDMA Network: Performance Analysis and Implementation Concepts, IEEE Trans. Commun. 63 (3) (2015) 882-891.

[143] M. V. Jamali, F. Akhoundi, J. A. Salehi, Performance characterization of relay-assisted wireless optical CDMA networks in turbulent underwater channel, IEEE Trans. Wireless Commun. 15 (2016) 4104-4116.

[144] F. Akhoundi, M. V. Jamali, N. B. Hassan, H. Beyranvand, A. Minoofar, J. A. Salehi, Cellular Underwater Wireless Optical CDMA Network: Potentials and Challenges, IEEE Access 4 (2016) 4254-4268.

[145] U. Nadeem, N. U. Hassan, M. A. Pasha, C. Yuen, Indoor positioning system designs using visible LED lights: performance comparison of TDM and FDM protocols, Electron. Lett. 51 (1) (2015) 72-74.

[146] Y. Hou, S. Xiao, H. Zheng, W. Hu, Multiple access scheme based on block encoding time division multiplexing in an indoor positioning system using visible light, IEEE J. Opt. Commun. Netw. 7 (5) (2015) 489_ 495.

[147] Y. Wang, N. Chi, Y. Wang, L. Tao, J. Shi, High speed LED based visible light communication networks for beyond $10 \mathrm{~Gb} / \mathrm{s}$ wireless access, in: Sixth Int. Conf. on Wireless Commun. and Signal Process., (WCSP), 2014, pp. 1-6.

[148] J. Armstrong, R. J. Green, M. D. Higgins, Comparison of Three Receiver Designs for Optical Wireless Communications using White LEDs, IEEE Commun. Lett. 16 (5) (2012) 748-751.

[149] H. Elgala, R. Mesleh, H. Haas, Indoor optical wireless communication: potential and state-of-the-art, IEEE Commun. Mag. 49 (9) (2011) 56-62.

[150] M. F. Sanya, L. Djogbe, A. Vianou, C. Aupetit-Berthelemot, DC-biased optical OFDM for IM/DD passive optical network systems, IEEE J. Opt. Commun. Netw. 7 (4) (2015) 205-214

[151] J. Tan, Z. Wang, Q. Wang, L. Dai, Near-Optimal Low-Complexity Sequence Detection for Clipped DCO-OFDM, IEEE Photon. Technol. Lett. 28 (3) (2016) 233-236.

[152] H. Zhang, Y. Yuan, W. Xu, PAPR Reduction for DCO-OFDM Visible Light Communications via Semidefinite Relaxation, IEEE Photon. Technol. Lett. 26 (17) (2014) 1718-1721.

[153] Z. Yu, R. J. Baxley, G. T. Zhou, Iterative Clipping for PAPR Reduction in Visible Light OFDM Communications, in: IEEE Military Commun. Conf., (MILCOM), 2014, pp. 1681-1686.

[154] X. Li, J. Vucic, V. Jungnickel, J. Armstrong, On the Capacity of Intensity-Modulated Direct-Detection Systems and the Information Rate of ACO-OFDM for Indoor Optical Wireless Applications, IEEE Trans. Commun. 60 (3) (2012) 799-809

[155] J. Dang, Z. Zhang, Comparison of optical OFDM-IDMA and optical ofdma for uplink visible light communications, in: Int. Conf. on Wireless Commun. and Signal Process., (WCSP), 2012, pp. 1-6.

[156] E. Dinc, O. Ergul, O. B. Akan, Soft handover in OFDMA based visible light communication networks, in: IEEE 82nd Vehicular Techno. Conf., (VTC), 2015, pp. 1-5.

[157] B. Lin, X. Tang, H. Yang, Z. Ghassemlooy, S. Zhang, Y. Li, C. Lin, Experimental demonstration of IFDMA for uplink visible light communication, IEEE Photon. Techno. Lett. 28 (20) (2016) 2218-2220.

[158] M. Jazayerifar, J. A. Salehi, Atmospheric optical CDMA communication systems via optical orthogonal codes, IEEE Trans. Commun. 54 (9) (2006) 1614-1623

[159] B. M. Ghaffari, M. D. Matinfar, J. A. Salehi, Wireless optical CDMA LAN: digital design concepts, IEEE Trans. Commun. 56 (12) (2008) 2145-2155.

[160] K. Fouli, M. Maier, OCDMA and optical coding: Principles, applications, and challenges, IEEE Commun. Mag. 45 (8) (2007) 27-34.

[161] A. Stok, E. H. Sargent, The role of optical CDMA in access networks, IEEE Commun. Mag. 40 (9) (2002) 83-87.

[162] H. Marshoud, V. M. Kapinas, G. K. Karagiannidis, S. Muhaidat, Nonorthogonal multiple access for visible light communications, IEEE Photon. Techno. Lett. 28 (1) (2016) 51-54

[163] H. Marshoud, P. C. Sofotasios, S. Muhaidat, G. K. Karagiannidis, B. S. Sharif, On the performance of visible light communication systems with non-orthogonal multiple access, IEEE Trans. Commun. 16 (10) (2017) 6350-6364.

[164] D. Tsonev, S. Videv, H. Haas, Light fidelity (li-fi): towards all-optical networking, Proc. SPIE 9007 (10) (2014) 1-10

[165] Z. Chen, H. Haas, Space division multiple access in visible light communications, in: IEEE Int. Conf. on Commun., (ICC), 2015, pp. 5115- 
5119

[166] T. Scholz, Laser based underwater communication experiments in the Baltic sea, Fourth Underwater Commun. and Netw. Conf., (UComms) (2018) 1-3.

[167] D. J. Richardson, J. M. Fini, L. E. Nelson, Space-division multiplexing in optical fibres, Nature Photonics 7 (2013) 354-362.

[168] A. E. Willner, Z. Zhao, Y. Ren, L. Li, G. Xie, H. Song, C. Liu, R. Zhang, C. Bao, K. Pang, Underwater optical communications using orbital angular momentum-based spatial division multiplexing, Optics Commun. 408 (2018) $21-25$.

[169] L. Allen, M. W. Beijersbergen, R. J. C. Spreeuw, J. P. Woerdman, Orbital angular momentum of light and the transformation of laguerre-gaussian laser modes, Phys. Rev. A 45 (1992) 8185-8189.

[170] J. Baghdady, M. Byrd, W. Li, K. Morgan, A. Pung, K. Miller, E. Johnson, Spatial multiplexing for blue lasers for undersea communications, Proc. SPIE 9459 (2015) 1-7.

[171] J. Baghdady, K. Miller, K. Morgan, M. Byrd, S. Osler, R. Ragusa, W. Li, B. M. Cochenour, E. G. Johnson, Multi-gigabit/s underwater optical communication link using orbital angular momentum multiplexing, Opt. Express 24 (9) (2016) 9794-9805.

[172] Y. Ren, L. Li, Z. Wang, S. M. Kamali, E. Arbabi, A. Arbabi, Z. Zhao, G. Xie, Y. Cao, N. Ahmed, C. L. Y. Yan, A. J. Willner, S. Ashrafi, M. Tur, A. Faraon, A. E. Willner, Orbital angular momentum-based space division multiplexing for high-capacity underwater optical communications, Sci. Rep. 6 (2016) 33306.

[173] Y. Zhao, J. Xu, A. Wang, W. Lv, L. Zhu, S. Li, J. Wang, Demonstration of data-carrying orbital angular momentum-based underwater wireless optical multicasting link, Opt. Express 25 (23) (2017) 28743-28751.

[174] Y. Zhao, C. Cai, J. Zhang, X. Cao, L. Wang, S. Li, J. Wang, Feedbackenabled adaptive underwater twisted light transmission link utilizing the reflection at the air-water interface, Opt. Express 26 (13) (2018) 1610216112

[175] A. Trichili, C. Rosales-Guzman, A. Dudley, B. Ndagano, A. B. Salem, M. Zghal, A. Forbes, Optical communication beyond orbital angular momentum, Sci. Rep. 6 (2016) 27674.

[176] A. Trichili, K.-H. Park, M. Zghal, B. S. Ooi, M.-S. Alouini, Communicating using spatial mode multiplexing: Potentials, challenges and perspectives, arXiv preprint arXiv:1808.02462.

[177] M. A. Khalighi, M. Uysal, Survey on Free Space Optical Communication: A Communication Theory Perspective, IEEE Commun. Surveys Tuts. 16 (4) (2014) 2231-2258

[178] M. Al-Bzoor, Y. Zhu, J. Liu, R. Ammar, J.-H. Cui, S. Rajasekaran, An adaptive power controlled routing protocol for underwater sensor network, Int. J. Sensor Networks 18 (4) (2015) 238-249.

[179] T.-H. Ho, S. Trisno, I. I. Smolyaninov, S. D. Milner, C. C. Davis, Studies of pointing, acquisition, and tracking of agile optical wireless transceivers for free-space optical communication networks, Proc. SPIE 5237 (12) (2004) 1 - 13.

[180] V. Nikulin, R. Khandekar, J. Sofka, Performance of a laser communication system with acousto-optic tracking: an experimental study, Proc. SPIE 6105 (10) (2006) 1-10.

[181] P. LoPresti, H. Refai, J. Sluss, I. Varela-Cuadrado, Adaptive divergence and power for improving connectivity in free-space optical mobile networks, Appl. Opt. 45 (25) (2006) 6591-6597.

[182] M. Uysal, M. Uysal, Cooperative Communications for Improved Wireless Network Transmission: Framework for Virtual Antenna Array Applications, Information Science Reference - Imprint of: IGI Publishing, Hershey, PA, 2009.

[183] N. D. Chatzidiamantis, D. S. Michalopoulos, E. E. Kriezis, G. K. Karagiannidis, R. Schober, Relay selection protocols for relay-assisted freespace optical systems, J. Opt. Commun. Netw. 5 (1) (2013) 92-103.

[184] N. Saeed, A. Celik, T. Y. Al-Naffouri, M.-S. Alouini, Localization of energy harvesting empowered underwater optical wireless sensor networks, IEEE Trans. Wireless Commun. 18 (5) (2019) 2652-2663.

[185] S. Kazemlou, S. Hranilovic, S. Kumar, All-optical multihop free-space optical communication systems, J. of Lightwave Techno. 29 (18) (2011) 2663-2669.

[186] M. Karimi, M. Nasiri-Kenari, BER analysis of cooperative systems in free-space optical networks, J. of Lightwave Techno. 27 (24) (2009) 5639-5647.

[187] M. Ayaz, I. Baig, A. Abdullah, I. Faye, A survey on routing techniques in underwater wireless sensor networks, J. of Netw. and Computer App. 34 (6) (2011) 1908 - 1927.

[188] R. Otnes, A. Asterjadhi, P. Casari, M. Goetz, T. Husoy, I. Nissen, K. Rimstad, P. van Walree, M. Zorzi, Edited by, in: J. R. Apel (Ed.), Underwater Acoustic Networking Techniques, Vol. 1, Springer-Verlag Berlin Heidelberg, 2012, pp. 1 - 83.

[189] N. Li, J.-F. Martinez, J. M. M. Chaus, M. Eckert, A Survey on Underwater Acoustic Sensor Network Routing Protocols, Sensors 16 (3) (2016) $1-28$.

[190] M. Ahmed, M. Salleh, M. I. Channa, Routing protocols for underwater wireless sensor networks based on data forwarding: a review, Telecommun. Systems 65 (1) (2017) 139-153.

[191] Q. Lu, F. Liu, Y. Zhang, S. Jiang, Routing protocols for underwater acoustic sensor networks: A survey from an application perspective, in: Advances in Underwater Acoustics, InTech, Rijeka, 2017.

[192] S. Sahana, K. Singh, R. Kumar, S. Das, A review of underwater wireless sensor network routing protocols and challenges, in: Next-Generation Networks, 2018, pp. 505-512.

[193] R. Alghamdi, N. Saeed, H. Dahrouj, T. Y. Al-Naffouri, M. Alouini, On distributed routing in underwater optical wireless sensor networks, CoRR abs/1811.05308. arXiv:1811.05308. URL http: //arxiv.org/abs/1811.05308

[194] A. Celik, N. Saeed, B. Shihada, T. Y. Al-Naffouri, M.-S. Alouini, SectOR: Sector-based opportunistic routing protocol for underwater optical wireless networks, in: IEEE Wireless Commun. and Netw. Conf., (WCNC), 2019, pp. 1-6.

[195] S. Hirai, Y. Tanigawa, H. Tode, Integration method between localization and routing in underwater sensor network, in: IEEE 15th Int. Conf. on Computational Sci. and Eng., 2012, pp. 689-693.

[196] Y. Tanigawa, S. Hirai, H. Tode, Lightweight data transfer unified with active localization and robust routing in underwater networks, in: IEEE Int. Conf. on Commun., (ICC), 2015, pp. 2534-2539.

[197] P. Wang, D. h. Fu, C. q. Zhao, J. c. Xing, Q. 1. Yang, X. f. Du, A reliable and efficient routing protocol for underwater acoustic sensor networks, in: IEEE Int. Conf. on Cyber Techno. in Auto., Cont. and Intelligent Sys., 2013, pp. 185-190.

[198] D. Hwang, D. Kim, DFR: Directional flooding-based routing protocol for underwater sensor networks, in: OCEANS, 2008, pp. 1-7.

[199] S. H. Ahmed, S. Lee, J. Park, D. Kim, D. B. Rawat, iDFR: Intelligent directional flooding-based routing protocols for underwater sensor networks, in: 14th IEEE Annual Consumer Commun. Netw. Conf., (CCNC), 2017, pp. 560-565.

[200] J. M. Jornet, M. Stojanovic, M. Zorzi, Focused beam routing protocol for underwater acoustic networks, in: Proc. of the Third ACM Int. Workshop on Underwater Netw., 2008, pp. 75-82.

[201] L. Emokpae, M. Younis, Signal reflection-enabled geographical routing for underwater sensor networks, in: IEEE Int. Conf. on Commun., (ICC), 2012, pp. 147-151.

[202] N. Chirdchoo, W. S. Soh, K. C. Chua, Sector-based routing with destination location prediction for underwater mobile networks, in: Int. Conf. on Adv. Info. Netw. and App. Workshops, 2009, pp. 1148-1153.

[203] S. Zhang, D. Li, A beam width and direction concerned routing for underwater acoustic sensor networks, in: IEEE 9th Int. Conf. on Mobile Ad-hoc and Sensor Netw., 2013, pp. 17-24.

[204] Y. Wei, D. S. Kim, Reliable and energy-efficient routing protocol for underwater acoustic sensor networks, in: Int. Conf. on Info. and Commun. Techno. Conv., (ICTC), 2014, pp. 738-743.

[205] E. Kim, J. Kang, P. K. Chong, S. Yoo, D. Kim, Energy efficient local area source routing protocol of underwater sensor networks in the deep ocean, in: Int. Symp. on Commun. and Info. Techno., 2007, pp. 948953.

[206] M. Al-Bzoor, Y. Zhu, J. Liu, A. Reda, J.-H. Cui, S. Rajasekaran, Adaptive power controlled routing for underwater sensor networks, in: X. Wang, R. Zheng, T. Jing, K. Xing (Eds.), Wireless Algorithms, Systems, and Applications, 2012, pp. 549-560.

[207] Y. D. Chen, Y. W. Chen, C. Y. Lien, K. P. Shih, A channel-aware depthadaptive routing protocol for underwater acoustic sensor networks, in: OCEANS, 2014, pp. 1-6.

[208] X. Chen, G. Lin, Energy efficient routing protocol for underwater acoustic networks using shortest path algorithm, in: 4th Int. Conf. on Info. Sci. and Cont. Eng., (ICISCE), 2017, pp. 295-300. 
[209] Y. D. Chen, C. Y. Lien, C. H. Wang, K. P. Shih, DARP: A depth adaptive routing protocol for large-scale underwater acoustic sensor networks, in: OCEANS, 2012, pp. 1-6.

[210] R. W. L. Coutinho, L. F. M. Vieira, A. A. F. Loureiro, DCR: DepthControlled Routing protocol for underwater sensor networks, in: IEEE Symp. on Computers and Commun., (ISCC), 2013, pp. 453-458.

[211] L. C. Kuo, T. Melodia, Cross-layer routing on MIMO-OFDM underwater acoustic links, in: Annual IEEE Commun. Soc. Conf. on Sensor, Mesh and Ad Hoc Commun. and Netw., (SECON), 2012, pp. 227-235.

212] X. Wu, G. Chen, J. Chen, Energy-Efficient and Topology-Aware Routing for Underwater Sensor Networks, in: Proc. of 19th Int. Conf. on Computer Commun. and Netw., 2010, pp. 1-6.

[213] Z. Guo, G. Colombi, B. Wang, J. H. Cui, D. Maggiorini, G. P. Rossi, Adaptive Routing in Underwater Delay/Disruption Tolerant Sensor Networks, in: Fifth Annual Conf. on Wireless on Demand Netw. Sys. and Serv., 2008, pp. 31-39.

[214] D. Pompili, T. Melodia, I. F. Akyildiz, Routing algorithms for delayinsensitive and delay-sensitive applications in underwater sensor networks, in: Proc. of the 12th Annual Int. Conf. on Mobile Computing and Netw., 2006, pp. 298-309.

[215] M. Goetz, I. Nissen, GUWMANET Multicast routing in Underwater Acoustic Networks, in: Military Commun. and Info. Sys. Conf., (MCC), 2012, pp. 1-8.

[216] Z. Zhou, Z. Peng, J. H. Cui, Z. Shi, Efficient multipath communication for time-critical applications in underwater acoustic sensor networks, IEEE/ACM Trans. Netw. 19 (1) (2011) 28-41.

[217] J. Xu, K. Li, G. Min, Reliable and energy-efficient multipath communications in underwater sensor networks, IEEE Trans. Parallel Distrib. Syst. 23 (7) (2012) 1326-1335

[218] S. Basagni, C. Petrioli, R. Petroccia, D. Spaccini, Channel-aware routing for underwater wireless networks, in: OCEANS, 2012, pp. 1-9.

[219] K. R. Anupama, A. Sasidharan, S. Vadlamani, A location-based clustering algorithm for data gathering in 3D underwater wireless sensor networks, in: Int. Symp. on Telecommun., 2008, pp. 343-348.

[220] C. Wang, G. Liu, LUM-HEED: A Location Unaware, Multi-hop routing protocol for Underwater Acoustic Sensor Networks, in: Proc. of Int. Conf. on Computer Sci. and Netw. Techno., Vol. 4, 2011, pp. 23362340.

[221] T. Hu, Y. Fei, An adaptive routing protocol based on connectivity prediction for underwater disruption tolerant networks, in: IEEE Global Commun. Conf., (GLOBECOM), 2013, pp. 65-71.

[222] T. Hu, Y. Fei, QELAR: A Machine-Learning-Based Adaptive Routing Protocol for Energy-Efficient and Lifetime-Extended Underwater Sensor Networks, IEEE Trans. Mobile Comput. 9 (6) (2010) 796-809.

[223] S. Ibrahim, J.-H. Cui, R. Ammar, Efficient surface gateway deployment for underwater sensor networks, in: IEEE Symp. on Computers and Commun. (ISCC), 2008, pp. 1177-1182.

[224] H. Alhumyani, R. Ammar, H. Albarakati, A. Alharbi, Deployment strategies for underwater sensing and processing networks, in: IEEE Symp. on Computers and Commun. (ISCC), 2016, pp. 358-363.

[225] S. Ibrahim, R. Ammar, J. H. Cui, Geometry-assisted gateway deployment for underwater sensor networks, in: IEEE Symp. on Computers and Commun. (ISCC), 2009, pp. 932-937.

[226] S. Ibrahim, J. Liu, M. Al-Bzoor, J.-H. Cui, R. Ammar, Towards efficient dynamic surface gateway deployment for underwater network, Ad Hoc Networks 11 (8) (2013) $2301-2312$.

[227] M. Al-Bzoor, R. Ammar, J. H. Cui, S. Rajasekaran, Coordinated multisurface gateway redeployment for enhanced performance in underwater sensor networks, in: IEEE Symp. on Computers and Commun. (ISCC), 2014, pp. 1-6.

[228] M. Al-Bzoor, Y. Zhu, J. Liu, R. Ammar, J.-H. Cui, S. Rajasekaran, An adaptive surface sink redeployment strategy for underwater sensor networks, in: IEEE Symp. on Computers and Commun. (ISCC), 2013, pp. 801-806.

[229] H. Albarakati, A. Amamra, R. Elfouly, R. Ammar, Reconfigurable underwater embedded systems architectures, in: IEEE Symp. on Computers and Commun. (ISCC), 2017, pp. 1372-1379.

[230] S. Ibrahim, R. Ammar, J. H. Cui, Surface gateway placement strategy for maximizing underwater sensor network lifetime, in: IEEE Symp. on Computers and Commun. (ISCC), 2010, pp. 342-346.

[231] M. Khalid, Z. Ullah, N. Ahmad, H. Khan, H. S. Cruickshank, O. U.
Khan, A comparative simulation based analysis of location based routing protocols in underwater wireless sensor networks, in: 2nd Workshop on Recent Trends in Telecommun. Res., (RTTR), 2017, pp. 1-5.

[232] M. C. Domingo, R. Prior, Design and analysis of a gps-free routing protocol for underwater wireless sensor networks in deep water, in: Int. Conf. on Sensor Techno. and App., (SENSORCOMM), 2007, pp. 215 220.

[233] D. N. Sandeep, V. Kumar, Review on Clustering, Coverage and Connectivity in Underwater Wireless Sensor Networks: A Communication Techniques Perspective, IEEE Access 5 (2017) 11176-11199.

[234] T. Hu, Y. Fei, MURAO: A multi-level routing protocol for acousticoptical hybrid underwater wireless sensor networks, in: 9th Annual IEEE Commun. Soc. Conf. on Sensor, Mesh and Ad Hoc Commun. and Netw., (SECON), 2012, pp. 218-226.

[235] R. W. L. Coutinho, A. Boukerche, L. F. M. Vieira, A. A. F. Loureiro, Modeling the sleep interval effects in duty-cycled underwater sensor networks, in: IEEE Int. Conf. on Commun., (ICC), 2016, pp. 1-6.

[236] V. Rodoplu, M. K. Park, An energy-efficient MAC protocol for underwater wireless acoustic networks, in: Proc. of OCEANS, 2005, pp. 1198 1203 Vol. 2.

[237] F. Zorzi, M. Stojanovic, M. Zorzi, On the effects of node density and duty cycle on energy efficiency in underwater networks, in: Proc. of OCEANS, 2010, pp. 1-6.

[238] R. W. Coutinho, A. Boukerche, L. F. Vieira, A. A. Loureiro, Modeling and analysis of opportunistic routing in low duty-cycle underwater sensor networks, in: Proc. of the 18th ACM Int. Conf. on Modeling, Analysis and Simulation of Wireless and Mobile Systems, 2015, pp. 125-132.

[239] S. Sudevalayam, P. Kulkarni, Energy harvesting sensor nodes: Survey and implications, IEEE Commun. Surveys Tuts. 13 (3) (2011) 443-461.

[240] H. Wang, J.-D. Park, Z. J. Ren, Practical energy harvesting for microbial fuel cells: A review, Env. Sci. \& Techno. 49 (6) (2015) 3267-3277.

[241] J. Dall, M. Christensen, Random geometric graphs, Phys. Rev. E 66 (2002) 16-21.

[242] P. Erdos, A. Renyi, On the evolution of random graphs, in: Publication of the mathematical institute of the Hungarian academy of sciences, 1960, pp. 17-61.

[243] G. Cossu, R. Corsini, E. Ciaramella, High-speed bi-directional optical wireless system in non-directed line-of-sight configuration, J. Lightwave Technol. 32 (10) (2014) 2035-2040.

[244] X. Li, J. Yu, Large-capacity long-distance bidirectional wireless signal transmission at hybrid K-and W-band, in: Broadband Access Commun. Techno., Vol. 10559, 2018, p. 105590R.

[245] C. Bettstetter, On the connectivity of ad hoc networks, The Computer J. 47 (4) (2004) 432-447.

[246] Z. Liao, D. Li, J. Chen, A network access mechanism for multihop underwater acoustic local area networks, IEEE Sensors J. 16 (10) (2016) 3914-3926.

[247] H. AlQuwaiee, I. S. Ansari, M. S. Alouini, On the performance of freespace optical communication systems over double generalized gamma channel, IEEE J. Sel. Areas Commun. 33 (9) (2015) 1829-1840.

[248] P. Gupta, P. R. Kumar, Critical power for asymptotic connectivity, in: Proc. of the 37th IEEE Conf. on Decision and Control, Vol. 1, 1998, pp. 1106-1110.

[249] C. Y. Wu, C. W. Yu, Computing the probability of specific random sector graphs with applications in wireless networks with directional antennas, in: Tenth Int. Conf. on Intelligent Info. Hiding and Multimedia Signal Process., 2014, pp. 678-681.

[250] A. Vavoulas, H. G. Sandalidis, D. Varoutas, Underwater optical wireless networks: A $k$-connectivity analysis, IEEE J. Ocean. Eng. 39 (4) (2014) 801-809.

[251] N. Saeed, A. Celik, T. Y. Al-Naffouri, M.-S. Alouini, Connectivity analysis of underwater optical wireless sensor networks: A graph theoretic approach, in: Proc. of IEEE Int. Conf. on Commun., (ICC), 2018, pp. $1-5$.

[252] N. Saeed, A. Celik, S. Alouini, T. Y. Al-Naffouri, Performance analysis of connectivity and localization in multi-hop underwater optical wireless sensor networks, IEEE Trans. on Mobile Comput. (2018) 1-1doi:10. 1109/TMC. 2018. 2878672.

[253] P. Gupta, P. R. Kumar, Critical power for asymptotic connectivity in wireless networks, in: W. M. McEneaney, G. G. Yin, Q. Zhang (Eds.), Stochastic Analysis, Control, Optimization and Applications: A Volume 
in Honor of W.H. Fleming, Birkhäuser Boston, Boston, MA, 1999, pp. 547-566.

[254] P. Gupta, P. R. Kumar, The capacity of wireless networks, IEEE Trans. Inf. Theory 46 (2) (2000) 388-404

[255] A. Ozgur, O. Leveque, D. Tse, Hierarchical cooperation achieves linear capacity scaling in ad hoc networks, in: Proc. IEEE Int. Conf. on Computer Commun. (INFOCOM), 2007, pp. 382-390.

[256] F. A. Onat, I. Stojmenovic, H. Yanikomeroglu, Generating random graphs for the simulation of wireless ad hoc, actuator, sensor, and internet networks, Perv. and Mobile Comp. 4 (5) (2008) 597-615.

[257] H. M. Ammari, S. K. Das, Critical density for coverage and connectivity in three-dimensional wireless sensor networks using continuum percolation, IEEE Trans. Parallel Distrib. Syst. 20 (6) (2009) 872-885.

[258] H. M. Ammari, Challenges and Opportunities of Connected k-Covered Wireless Sensor Networks, Springer-Verlag, Berlin Heidelberg, 2009.

[259] J. P. Coon, O. Georgiou, C. P. Dettmann, Connectivity scaling laws in wireless networks, IEEE Wireless Commun. Lett. 4 (6) (2015) 629-632.

[260] Y. Liu, J. Gao, J. Yu, C. Yin, Local connectivity for heterogeneous overlaid wireless networks, Ad Hoc Netw. 58 (Supplement C) (2017) 205 212.

[261] V. Ravelomanana, Extremal properties of three-dimensional sensor networks with applications, IEEE Trans. Mobile Comput. 3 (3) (2004) 246257

[262] K. Akkaya, A. Newell, Self-deployment of sensors for maximized coverage in underwater acoustic sensor networks, Computer Commun. 32 (710) (2009) 1233-1244.

[263] D. Pompili, T. Melodia, I. F. Akyildiz, Deployment analysis in underwater acoustic wireless sensor networks, in: Proc. of the 1st ACM Int. Workshop on Underwater Networks, ACM, New York, NY, USA, 2006, pp. $48-55$.

[264] F. Senel, K. Akkaya, M. Erol-Kantarci, T. Yilmaz, Self-deployment of mobile underwater acoustic sensor networks for maximized coverage and guaranteed connectivity, Ad Hoc Netw. 34 (Supplement C) (2015) $170-183$

[265] Q. Wang, H. N. Dai, C. F. Cheang, H. Wang, Link connectivity and coverage of underwater cognitive acoustic networks under spectrum constraint, Sensors 17 (12) (2017) 1-15.

[266] J. Diaz, J. Petit, M. Serna, A random graph model for optical networks of sensors, IEEE Trans. Mobile Comput. 2 (3) (2003) 186-196.

[267] D. Kundur, U. N. Okorafor, W. Luh, Holistic: Heterogeneous lightweight sensornets for trusted visual computing, in: Int. Conf. on Intelligent Info. Hiding and Multimedia, 2006, pp. 267-270.

[268] U. N. Okorafor, D. Kundur, On the connectivity of hierarchical directional optical sensor networks, in: IEEE Wireless Commun. and Netw. Conf., (WCNC), 2007, pp. 3524-3528.

[269] R. Xie, F. Tong, D. Kang, Y. C. Kim, A reconfigurable routing protocol for free space optical sensor network, in: The Int. Conf. on Info. Netw. (ICOIN), 2011, pp. 165-170

[270] R. Xie, L.-M. Peng, W. Tang, F. Tong, D. ki Kang, W.-H. Yang, Y.-C. Kim, A simulation study of neighborhood discovery algorithm in free space optical sensor networks, in: Second Int. Conf. on Ubiquitous and Future Networks (ICUFN), 2010, pp. 87-91.

[271] R. Ferrero, M. V. Bueno-Delgado, F. Gandino, In- and out-degree distributions of nodes and coverage in random sector graphs, IEEE Trans. Wireless Commun. 13 (4) (2014) 2074-2085

[272] N. Saeed, A. Celik, T. Y. Al-Naffouri, M.-S. Alouini, Underwater optical sensor networks localization with limited connectivity, in: Proc. of IEEE Int. Conf. on Acoustics, Speech and Signal Processing (ICASSP), 2018, pp. 1-5.

[273] M. J. B. Appel, R. P. Russo, The minimum vertex degree of a graph on uniform points in [0,1], Adv. in App. Probability 29 (3) (1997) 582-594.

[274] N. Saeed, A. Celik, T. Y. Al-Naffouri, M.-S. Alouini, Underwater optical wireless communications, networking, and localization: A survey, arXiv preprint arXiv: 1803.02442

[275] M. Erol-Kantarci, H. T. Mouftah, S. Oktug, Localization techniques for underwater acoustic sensor networks, IEEE Commun. Mag. 48 (12) (2010) 152-158.

[276] H.-P. Tan, R. Diamant, W. K. Seah, M. Waldmeyer, A survey of techniques and challenges in underwater localization, Ocean Engineering 38 (14) (2011) 1663 - 1676

[277] M. Erol-Kantarci, H. T. Mouftah, S. Oktug, A survey of architectures and localization techniques for underwater acoustic sensor networks, IEEE Commun. Surveys Tuts. 13 (3) (2011) 487-502.

[278] G. Tuna, V. C. Gungor, A survey on deployment techniques, localization algorithms, and research challenges for underwater acoustic sensor networks, Int. J. of Commun. Sys. 30 (17) (2017) 1-21.

[279] G. Berkovic, E. Shafir, Optical methods for distance and displacement measurements, Adv. Opt. Photon. 4 (4) (2012) 441-471

[280] R. Diamant, L. Lampe, Underwater localization with timesynchronization and propagation speed uncertainties, IEEE Trans. Mobile Comput. 12 (7) (2013) 1257-1269.

[281] G. Cario, A. Casavola, V. Djapic, P. Gjanci, M. Lupia, C. Petrioli, D. Spaccini, Clock synchronization and ranging estimation for control and cooperation of multiple UUVs, in: OCEANS, 2016, pp. 1-9.

[282] J. Crowell, Method and apparatus for synchronizing clocks underwater using light and sound, US Patent 9,645,272 (May 2017).

[283] M. Stojanovic, J. Preisig, Underwater acoustic communication channels: Propagation models and statistical characterization, IEEE Commun. Mag. 47 (1) (2009) 84-89.

284] M. Kanaan, K. Pahlavan, CN-TOAG: a new algorithm for indoor geolocation, in: IEEE 15th Int. Symp. on Personal, Indoor and Mobile Radio Commun., Vol. 3, 2004, pp. 1906-1910.

[285] L. Emokpae, M. Younis, Surface based anchor-free localization algorithm for underwater sensor networks, in: IEEE Int. Conf. on Commun., (ICC), 2011, pp. 1-5.

[286] P. A. van Walree, Propagation and scattering effects in underwater acoustic communication channels, IEEE J. Ocean. Eng. 38 (4) (2013) 614 631.

[287] X. Cheng, H. Shu, Q. Liang, D. H. C. Du, Silent positioning in underwater acoustic sensor networks, IEEE Trans. Veh. Technol. 57 (3) (2008) 1756-1766.

[288] S. Poursheikhali, H. Zamiri-Jafarian, TDOA based target localization in inhomogenous underwater wireless sensor network, in: 5th Int. Conf. on Computer and Knowledge Engineering (ICCKE), 2015, pp. 1-6.

[289] G. Isbitiren, O. B. Akan, Three-dimensional underwater target tracking with acoustic sensor networks, IEEE Trans. Veh. Technol. 60 (8) (2011) 3897-3906.

[290] J. Choi, H. T. Choi, Preliminary results on three dimensional localization of underwater acoustic sources, in: 14th Int. Conf. on Ubiquitous Robots and Ambient Intelligence (URAI), 2017, pp. 347-348.

[291] J. Scheuing, B. Yang, Disambiguation of TDOA estimation for multiple sources in reverberant environments, IEEE Trans. Acoust., Speech, Signal Process. 16 (8) (2008) 1479-1489.

[292] P. E. M. de Magalhães, C. Ioana, J. Mars, X. Cristol, Hausdorff distance applied on real data experiment for underwater localization, in: OCEANS, 2017, pp. 1-5.

[293] B. Kouzoundjian, F. Beaubois, S. Reboul, J. B. Choquel, J. C. Noyer, A TDOA underwater localization approach for shallow water environment, in: OCEANS, 2017, pp. 1-4.

[294] M. Hosseini, H. Chizari, A. S. Ismail, New hybrid RSS-based localization mechanism for underwater wireless sensor networks, Int. J. of Comp. Commun. and Netw. 1 (11) (2017) 1-10.

[295] T. Xu, Y. Hu, B. Zhang, G. Leus, Rss-based sensor localization in underwater acoustic sensor networks, in: IEEE Int. Conf. on Acoustics, Speech and Signal Processing (ICASSP), 2016, pp. 3906-3910.

[296] B. Zhang, H. Wang, T. Xu, L. Zheng, Q. Yang, Received signal strengthbased underwater acoustic localization considering stratification effect, in: OCEANS, 2016, pp. 1-8.

297] F. Akhoundi, A. Minoofar, J. A. Salehi, Underwater positioning system based on cellular underwater wireless optical cdma networks, in: Wireless and Opt. Commun. Conf., (WOCC), 2017, pp. 1-3.

[298] S. Pandey, P. Agrawal, A survey on localization techniques for wireless networks, J. of the Chinese Inst. of Engineers 29 (7) (2006) 1125-1148.

[299] G. Mao, B. Fidan, B. D. Anderson, Wireless sensor network localization techniques, Computer Netw. 51 (10) (2007) 2529 - 2553.

[300] A. R. Kulaib, R. M. Shubair, M. A. Al-Qutayri, J. W. P. Ng, An overview of localization techniques for wireless sensor networks, in: Int. Conf. on Innovations in Info. Techno., 2011, pp. 167-172.

[301] N. A. Alrajeh, M. Bashir, B. Shams, Localization techniques in wireless sensor networks, Int. J. of Dist. Sensor Netw. 9 (6) (2013) 304628

[302] J. Kuriakose, S. Joshi, R. Vikram Raju, A. Kilaru, A review on localization in wireless sensor networks, in: S. M. Thampi, A. Gelbukh, 
J. Mukhopadhyay (Eds.), Advances in Signal Processing and Intelligent Recognition Sys., Springer Int. Publishing, Cham, 2014, pp. 599-610.

[303] H. Khan, M. N. Hayat, Z. U. Rehman, Wireless sensor networks freerange base localization schemes: A comprehensive survey, in: Int. Con. on Commun., Comp. and Dig. Sys., (C-CODE), 2017, pp. 144-147.

[304] V. Chandrasekhar, W. K. Seah, Y. S. Choo, H. V. Ee, Localization in underwater sensor networks: Survey and challenges, in: Proc. of the 1st ACM Int. Workshop on Underwater Networks, 2006, pp. 33-40.

[305] J. Luo, L. Fan, S. Wu, X. Yan, Research on Localization Algorithms Based on Acoustic Communication for Underwater Sensor Networks, Sensors 18 (1) (2018) 1-25.

[306] R. M. Corless, G. H. Gonnet, D. E. G. Hare, D. J. Jeffrey, D. E. Knuth, On the lambertw function, Adv. in Comput. Math. 5 (1) (1996) 329-359.

[307] O. Korotkova, N. Farwell, E. Shchepakina, Light scintillation in oceanic turbulence, Waves in Random and Complex Media 22 (2) (2012) 260266.

[308] N. Saeed, A. Celik, T. Y. Al-Naffouri, M.-S. Alouini, Robust 3D localization of underwater optical wireless sensor networks via low rank matrix completion, in: Proc. of IEEE Int. Works. on Signal Processing Adv. in Wireless Commun. (SPAWC), 2018, pp. 1-5.

[309] N. Saeed, T. Y. Al-Naffouri, M. Alouini, Outlier detection and optimal anchor placement for 3-D underwater optical wireless sensor network localization, IEEE Trans. on Commun. 67 (1) (2019) 611-622.

[310] GODAE, Ocenview, Argo drifters, http://www.argo.ucsd.edu/, online; accessed 19 Dec. 2017.

[311] W. J. Gould, J. Turton, Argo-sounding the oceans, Weather 61 (1) (2006) $17-21$

[312] J. Griffiths, Beijing plans underwater observation system in south china sea, http://edition.cnn.com/2017/05/29/asia/ south-china-sea-underwater-observation-system/index . html, online; accessed 19 Dec. 2017.

[313] J. Rice, D. Green, Underwater acoustic communications and networks for the US navy's Seaweb program, in: Second Int. Conf. on Sensor Techno. and App., (sensorcomm), 2008, pp. 715-722.

[314] L. Collin, S. Azou, K. C. Yao, G. Burel, On spatial uncertainty in a surface long baseline positioning system, in: Fifth European Conf. on Underwater Acoustics, Lyon, France, 2000.

[315] B. Foley, D. Mindell, Precision survey and archaeological methodology in deep water, The J. of the Hellenic Inst.of Marine Arch. 6 (2002) 4956.

[316] R. D. Ballard, The MEDEA/JASON remotely operated vehicle system, Deep Sea Research Part I: Oceanographic Research Papers 40 (8) (1993) $1673-1687$

[317] J. R. Kayser, M. A. Cardoza, W. F. Wade, J. H. Merts, D. R. Casey, Weapon Scoring Results from a GPS Acoustic Weapons Test and Training System, in: Proc. of the 2005 National Technical Meeting of The Institute of Navigation, San Diego, CA, 2005.

[318] L. Chen, et al., Towards autonomous localization and mapping of AUVs: a survey, Int. J. of Intelligent Unmanned Sys. 1 (2) (2013) 97-120.

[319] Z. Zhou, J.-H. Cui, S. Zhou, Efficient localization for large-scale underwater sensor networks, Ad Hoc Netw. 8 (3) (2010) 267-279.

[320] E. Logics, Underwater acoustic LBL positioning sys., https://www. evologics.de/en/products/LBL/index.html, online; accessed 01 Jan. 2018.

[321] KONGSBERG, HiPAP - acoustic underwater positioning and navigation sys., https://www.km.kongsberg.com/ks/web/nokbg0240. nsf, online; accessed 01 Jan. 2018

[322] Sonardyne, Mini-ranger 2 underwater positioning (usbl) system, https://www. sonardyne.com/product/ underwater-positioning-mini-ranger-2/, online; accessed 01 Jan. 2018.

[323] iXblue, USBL positioning sys., https://www.ixblue.com/ products/usbl-positioning-Sys., online; accessed 01 Jan. 2018.

[324] K. Tech., Videoray ROV non-acoustic positioning system smart tether, https://www.videoray.com/homepage/new/options/ positioning-Sys./smart-tether.html, online; accessed 01 Jan. 2018.

[325] LinkQuest, Tracklink acoustic tracking and communication sys., http: //www.link-quest.com/html/intro2.htm, online; accessed 01 Jan. 2018.
[326] T. Marine, Teledyne benthos underwater acoustic sys., http://www. teledynemarine.com/positioning-Sys., online; accessed 01 Jan. 2018.

[327] A. Mora, D. Ganger, G. Wells, J. Zhang, X. Hu, C. Zhou, A. Richa, C. Youngbull, Ad-hoc multi-hop underwater optical network for deep ocean monitoring, in: OCEANS, 2013, pp. 1-5.

[328] M. V. Jamali, A. Chizari, J. A. Salehi, Performance analysis of multihop underwater wireless optical communication systems, IEEE Photon. Techno. Lett. 29 (5) (2017) 462-465.

[329] A. Tabeshnezhad, M. A. Pourmina, Outage analysis of relay-assisted underwater wireless optical communication systems, Opt. Commun. 405 (Supplement C) (2017) $297-305$.

[330] W. Wang, P. Wang, T. Cao, H. Tian, Y. Zhang, L. Guo, Performance investigation of underwater wireless optical communication system using M-ary OAMSK modulation over oceanic turbulence, IEEE Photon. J. 9 (5) (2017) 1-15.

[331] Y. Song, W. Lu, B. Sun, Y. Hong, F. Qu, J. Han, W. Zhang, J. Xu, Experimental demonstration of MIMO-OFDM underwater wireless optical communication, Opt. Commun. 403 (Supplement C) (2017) 205 - 210.

[332] R. Diamant, F. Campagnaro, M. de Filippo de Grazia, P. Casari, A. Testolin, V. S. Calzado, M. Zorzi, On the Relationship Between the Underwater Acoustic and Optical Channels, IEEE Trans. Wireless Commun. 16 (12) (2017) 8037-8051.

[333] H. Li, C. Tian, J. Lu, M. J. Myjak, J. J. Martinez, R. S. Brown, Z. D. Deng, An energy harvesting underwater acoustic transmitter for aquatic animals, Nature Scientific Reports 6 (2016) 33804.

[334] C.-C. Kao, Y.-S. Lin, G.-D. Wu, C.-J. Huang, A comprehensive study on the internet of underwater things: Applications, challenges, and channel models, Sensors 17 (1477) (2017) 1-20.

[335] Z. Zhou, B. Yao, R. Xing, L. Shu, S. Bu, E-CARP: An energy efficient routing protocol for UWSNs in the internet of underwater things, IEEE Sensors J. 16 (11) (2016) 4072-4082.

[336] N. Javaid, S. Cheema, M. Akbar, N. Alrajeh, M. S. Alabed, N. Guizani, Balanced energy consumption based adaptive routing for iot enabling underwater wsns, IEEE Access 5 (1) (2017) 40-51.

[337] M. Xu, L. Liu, Sender-receiver role-based energy-aware scheduling for internet of underwater things, IEEE Trans. Emerg. Topics Comput. (2018) 1-15.

[338] M. H. Berlian, T. E. R. Sahputra, B. J. W. Ardi, L. W. Dzatmika, A. R. A. Besari, R. W. Sudibyo, S. Sukaridhoto, Design and implementation of smart environment monitoring and analytics in real-time system framework based on internet of underwater things and big data, in: Int. Electronics Symp. (IES), 2016, pp. 403-408.

[339] F. Qu, H. Yang, G. Yu, L. Yang, In-Band Full-Duplex Communications for Underwater Acoustic Networks, IEEE Network 31 (5) (2017) 59-65.

[340] A. S. Thyagaturu, A. Mercian, M. P. McGarry, M. Reisslein, W. Kellerer, Software Defined Optical Networks (SDONs): A Comprehensive Survey, IEEE Commun. Surveys Tuts. 18 (4) (2016) 2738-2786.

[341] I. F. Akyildiz, P. Wang, S.-C. Lin, SoftWater: Software-defined networking for next-generation underwater communication systems, Ad Hoc Networks 46 (2016) 1 - 11 .

[342] I. F. Akyildiz, P. Wang, Z. Sun, Realizing underwater communication through magnetic induction, IEEE Commun. Mag. 53 (11) (2015) 42 48.

[343] Z. Sun, I. F. Akyildiz, Magnetic induction communications for wireless underground sensor networks, IEEE Trans. Antennas Propag. 58 (7) (2010) 2426-2435.

[344] S. C. Lin, I. F. Akyildiz, P. Wang, Z. Sun, Distributed cross-layer protocol design for magnetic induction communication in wireless underground sensor networks, IEEE Trans. Wireless Commun. 14 (7) (2015) 4006-4019.

[345] M. C. Domingo, Magnetic induction for underwater wireless communication networks, IEEE Trans. Antennas Propag. 60 (6) (2012) 2929 2939.

[346] B. Gulbahar, O. B. Akan, A communication theoretical modeling and analysis of underwater magneto-inductive wireless channels, IEEE Trans. Wireless Commun. 11 (9) (2012) 3326-3334.

[347] H. Guo, Z. Sun, P. Wang, Channel Modeling of MI Underwater Communication Using Tri-Directional Coil Antenna, in: IEEE Global Commun. Conf. (GLOBECOM), 2015, pp. 1-6.

[348] I. F. Akyildiz, P. Wang, Z. Sun, Realizing underwater communication 
through magnetic induction, IEEE Commun. Mag. 53 (11) (2015) 42-

48 . 